\title{
Proceedings of the British Cardiac Society (Golden Jubilee Meeting)
}

THE AUTUMN MEETING of the British Cardiac Society was held at the Wembley Conference Centre, London, on Tuesday to Thursday, 24 to 26 November 1987. The President, E sowton, took the Chair during private business. The scientific sessions were under the chairmanship of $\mathrm{D} N \mathrm{~N}$ ROss.

\section{Abstracts of papers}

Studies on ventricular fibrillation: spectral analysis and optimisation of defibrillation

\section{E J F Carlisle}

Regional Medical Cardiology Centre, Royal Victoria Hospital, Belfast

Fast Fourier transform analysis was used to study the ventricular fibrillation waveform in greyhounds and in man. The dominant frequency of canine fibrillation recorded from a surface lead remained over $9 \mathrm{~Hz}$ for 70 seconds, and then rapidly fell to about $5 \mathrm{~Hz}$; the time courses of fibrillation induced by acute myocardial ischaemia and reperfusion were similar. Fibrillation induced by administration of ouabain or potassium was significantly slower. The time courses of fibrillation recorded from the body surface and from the epicardium of the heart were similar, but fibrillation recorded from the endocardium did not show the rapid fall in frequency after one minute. The dominant frequency of ventricular fibrillation due to acute myocardial infarction in man $(6 \cdot 4(0 \cdot 2) \mathrm{Hz})$ was lower than in the $\operatorname{dog}(12 \cdot 3(0 \cdot 2) \mathrm{Hz})$. In dogs, pretreatment with lignocaine significantly reduced the dominant frequency of electrically induced ventricular fibrillation. Pretreatment with verapamil prevented the rapid fall in frequency seen after one minute in the untreated animals. Synchronisation of the defibrillation countershock to the peak or trough of the fibrillation waveform was possible, but appeared to be of no clinical value.

Antiarrhythmic drugs have significant effects during ventricular fibrillation. It is possible that calcium antagonist drugs may have a role in resuscitation from prolonged fibrillation.
Epicardial repolarisation sequence and $T$ wave configuration

J C Cowan

Department of Cardiology, Freeman Hospital, Newcastle upon Tyne

The association of ventricular repolarisation sequence and $T$ wave configuration in man is unclear. Epicardial activation and repolarisation sequences were studied in 19 patients, 14 under going coronary artery bypass grafting (upright $T$ waves) and five undergoing aortic valve replacement (four patients $T$ inversion). In patients with upright $T$ waves, an inverse relation was observed between monophasic action potential duration and activation time. As a consequence, activation and repolarisation proceeded in opposite directions-mean activation sequence was from septum to free wall and from apex to base, whereas mean repolarisation sequence was from base to apex and from free wall to septum. Dispersion of repolarisation (14 ms) was less than dispersion of activation ( $23 \mathrm{~ms})$. In patients with $T$ wave inversion due to aortic stenosis, there was no association between action potential duration and activation time; repolarisation sequence resembled activation sequence and dispersion of repolarisation was greater than dispersion of activation ( 31 and 26 $\mathrm{ms}$, respectively). Four patients underwent ventricular pacing to induce $\mathrm{T}$ wave discordance; repolarisation sequence was determined by activation sequence and dispersion of repolarisation increased.

The normal concordance of $Q R S$ and $T$ waves of the surface ECG is due to an inverse relation between action potential duration and activation time. This relation reduces dispersion of repolarisation and may be a fundamental intrinsic antiarrhythmic mechanism.

Development of an implantable method for rate independent recognition of cardiac rhythm

D W Davies

Department of Cardiology, St Bartholomew's Hospital, London 
The reliance of implantable pulse generators upon heart rate analysis for diagnosing the underlying cardiac rhythm leads to errors of both inappropriate pacing and unwanted witholding of pacing. A method for diagnosing the heart's rhythm by analysing intracardiac electrogram morphology has been developed. Detecting the pattern of the slopes or gradients of which electrograms are composed after digitisation and first differentiation, it has been refined from a system which analysed recorded signals to an economical program of less than 500 bytes. This program (gradient pattern detection) reliably detects arrhythmias at atrial and ventricular level in real time and can vary the response to up to four different arrhythmias in one patient. Variations in heart rate, respiration, and posture do not significantly affect the recognition process, but electrode site may be crucial.

At selected endocardial sites, analysis of electrogram configuration by gradient pattern detection operates at sufficient speed and with sufficient reliability to supplement heart rate analysis in implantable pulse generators.

Assessment of left ventricular performance during percutaneous transluminal coronary angioplasty (PTCA) using intravenous digital subtraction ventriculography

\section{S Norell \\ Cardiac Department, London Chest Hospital, London}

Left ventricular (LV) performance was studied in 52 patients during PTCA. Digital subtraction left ventriculography, after right atrial contrast injection, was performed before and during balloon coronary occlusion, and after the procedure in 37 patients. Twelve lead ECG was monitored throughout. During balloon inflation $\mathrm{LV}$ ejection fraction (EF) decreased in all but one patient (mean LVEF $73 \%$ to $57 \%, \mathrm{p}<0.001$ ), although ST segment alteration $(>1 \mathrm{~mm})$ developed in only 33 . The fall in LVEF correlated with the magnitude of both ST segment elevation $(r=0.637, p<0.001)$ and ST depression $(r=0.396, p<0.01)$. The decrease in LVEF was similar in patients with single and multivessel disease $(16 \%$ and $17 \%, \mathrm{NS})$, and was not different during 20 and 60 second balloon occlusions $(16 \%$ and $15 \%$, NS). LV end systolic volume (ESV) increased (44 to $76 \mathrm{ml}, \mathrm{p}<0.001$ ) while end diastolic volume did not change (140 to $148 \mathrm{ml}, \mathrm{NS}$ ). In contrast, mean $R$ wave amplitude fell $(10.1$ to $8.9 \mathrm{~mm}, \mathrm{p}<0.001)$. There was an inverse correlation between change in LVESV and $R$ wave amplitude $(r=-0.371$, $p<0.05)$. In patients undergoing single LAD PTCA
( $n=27)$, the fall in LVEF in the 15 without collateral vessels $(23 \%)$ was greater than that in the 12 patients with collaterals $(12 \%, \mathrm{p}<0.01)$. There was an inverse correlation between the degree of collateral supply and both the fall in LVEF $(\mathrm{r}=-0.446, \mathrm{p}<0.01)$, and the magnitude of ST alteration (ST elevation: $r=-0.680, p<0.001$; $S T$ depression: $r=-0.444, p<0.025)$. Both LVEF and regional wall motion returned to baseline values after the procedure.

Intravenous digital subtraction ventriculography is a sensitive method of examining $\mathrm{LV}$ performance during PTCA. In addition to showing the protective role of collateral vessels, it may be used to assess techniques designed to reduce myocardial ischaemia during balloon coronary occlusion.

Effect of physical training on blood pressure and baroreflex sensitivity in borderline hypertensive subjects

\section{J Somers \\ Department of Cardiovascular Medicine, John Radcliffe Hospital, Oxford}

The effect of physical training on blood pressure is controversial. Little is known of the effects of training on baroreflex sensitivity. Sixteen borderline hypertensive subjects were studied. Each subject was studied twice. Eight subjects were studied before and after a six month training programme, and the other eight after training, and then after a four month detraining period (loss of fitness). Measurements were taken of baroreflex sensitivity and intra-arterial blood pressure at rest, during mental arithmetic, during isometric exercise, and during dynamic exercise. Thirteen subjects also underwent ambulatory blood pressure measurement. For all 16 subjects increased fitness was associated with a fall in resting blood pressure from $148(1 \cdot 0) / 82(0 \cdot 7) \mathrm{mm} \mathrm{Hg}$ to $138(0.8) / 76(0.8) \mathrm{mm} \mathrm{Hg}(\mathrm{p}<0.001)$. Baroreflex sensitivity rose from $14.0(1.8)$ to $17 \cdot 5(2 \cdot 0) \mathrm{mg} / \mathrm{mm}$ $\mathrm{Hg}(\mathrm{p}<0.05)$ with increased fitness. Fitness did not, however, attenuate the blood pressure response to mental stress, resulting in a greater absolute blood pressure increase. Fitness did result in lower diastolic pressures during isometric and dynamic exercise. With increased fitness ambulatory blood pressure fell from $141(3.0) / 89(1 \cdot 6) \mathrm{mm} \mathrm{Hg}$ to $136(3.3) / 81(2.9) \mathrm{mm} \mathrm{Hg}(\mathrm{p}<0.01)$. These changes in blood pressure could not be explained by changes in weight or sodium or potassium intake.

We conclude that physical conditioning is an effective approach in the management of borderline hypertension. 
Intracellular calcium release initiates spontaneous calcium transients in vascular smooth muscle

\section{P L Weissberg}

Baker Medical Research Institute, Melbourne, Australia

Fluorescence measurements of calcium indicator signals (Fura 2 and Quin 2) from aortic smooth muscle cells in primary culture have shown spontaneous transient increases in intracellular calcium concentration. The transients consist of a rapid increase in intracellular calcium concentration of approximately $100 \mathrm{nmol} / \mathrm{l}$ which is sustained for approximately 30 seconds. The frequency of the transients can be modulated by low concentrations of vasoactive substances. Experiments are described which show that the transients are due to a spontaneous release of calcium from the sarcoplasmic reticulum followed by an influx of calcium through voltage dependent channels. No spontaneous influx occurs in the absence of an initial intracellular release of calcium. These findings are the first to show that: (a) the sarcoplasmic reticulum can spontaneously release calcium into the cytoplasm in the absence of receptor stimulation, and (b) that this release can initiate activation of voltage dependent channels. The findings support the view that the sarcoplasmic reticulum is the major system controlling cytoplasmic calcium in vascular smooth muscle cells and they provide a biochemical explanation for the spontaneous contractions frequently observed in intact vessels, including human coronary arteries.

This mechanism probably has a fundamental role in the regulation of vascular resistance and the development of coronary spasm.

\section{Intravenous and intrapulmonary recombinant tissue type plasminogen activator (rt-PA) in treatment of acute, massive, pulmonary embolism: a multicentre pilot study}

M Verstraete, G. A H Miller, H Bounameaux, B Charbonnier, J P Colle, G Lecorf, G A Marbet, P Mombaerts, C G Olsson

Brompton Hospital Representing the European Cooperative Study Group

Eight centres participated in a pilot study which compared intrapulmonary (PA) with intravenous (IV) treatment by recombinant tissue type plasminogen activator (rt-PA) in 34 patients with angiographically proved acute ( $<5$ days), massive, pulmonary embolism. All patients received intravenous heparin in a bolus of 5000 IU followed by $1000 \mathrm{IU} / \mathrm{h}$. After $50 \mathrm{mg}$ rt-PA given over two hours the severity of embolism, determined from pulmonary angiograms, declined by $12 \%$ in the PA group and $15 \%$ in the IV group. After a further 50 mg given over five hours (22 patients), the angiographically determined severity had decreased by $38 \%$ from baseline in the PA group and by $38 \%$ in the IV group. Measured haemodynamic variables showed major improvement with normalisation of pulmonary artery pressure after a seven hour treatment with $100 \mathrm{mg}$ rt-PA. Fibrinogen concentrations dropped $46 \%$ from baseline after $50 \mathrm{mg}$ and $66 \%$ from baseline after $100 \mathrm{mg} \mathrm{rt}-\mathrm{PA}$. Minor bleeding, most often limited to puncture sites, was noted in 12 patients. Four other patients, however, required a transfusion of two or more units of blood; all of them had been operated on an average of seven days (range 2-13) before thrombolytic treatment was started. In seven other patients thrombolytic treatment was started a mean of 8.5 days (range 3-15) after operation and no bleeding was seen.

In a dose of $100 \mathrm{mg}$ rt-PA results in appreciable lysis of pulmonary emboli. This effect is seen within eight hours of the start of treatment and intravenous administration was as effective as intrapulmonary. rt-PA is only relatively fibrin selective but there was major bleeding in only $12 \%$ of patients.

The benefit of very early intravenous streptokinase administration in acute myocardial infarction

\section{L Stone, A T Weiss, H Atlan, M S Gotsman Hadassah Hospital, Ein Kerem, Jerusalem, Israel}

Fifty one patients (age range 38-73; mean $54 \cdot 2$ years) who had received early intravenous streptokinase were divided into two groups: those whose pain before streptokinase lasted less than 90 minutes (group A: 23 patients) and those whose pain lasted 90 minutes to 4 hours (group B: 28 patients). Exercise thallium scintigrams were performed following discharge and scored visually in nine regions $(0=$ no defect, 3 = severe defect). In group A the myocardial defect score was lower (mean: group A 4.7(0.6); group B 6.6(0.7); p < 0.05) as was the infarct related zone score (group A 1.3(0.2); group B 2.1(0.3); p < $0.05)$. Thirty two of the patients were also assessed by a quantitative circumferential profile technique. The severity index was the area between the patients profile and normal. The total myocardial severity index (group A 559(81); group B 992(164); p < 0.03) and the infarct related severity index (group $A$ 
797(496); group B 1616(277), p<0.01) were also significantly lower in group A.

Exercise thallium scintigraphy showed that there was an improvement in patients who received early streptokinase for myocardial infarction and emphasised the need for the administration of this agent as soon as possible after the onset of pain.

\section{Reperfusion arrhythmias are uncommon during thrombolysis in acute evolving myocardial infarction}

\section{Hackett, Trudy Lowe, G Davies, W J McKenna, A Maseri \\ Royal Postgraduate Medical School, Hammersmith Hospital, London}

Arrhythmias are commonly associated with reperfusion in animal studies but the clinical relevance remains uncertain. We performed continuous frequency modulated electrocardiographic recordings immediately after admission to Casualty in $\mathbf{3 8}$ of $\mathbf{4 5}$ consecutive patients presenting within four (mean $2 \cdot 5$ ) hours of the onset of acute myocardial infarction and who had emergency coronary arteriography and intracoronary thrombolysis with streptokinase. All patients received diamorphine and high doses of nitrates but no prophylactic antiarrhythmic drugs. In 19 patients 36 episodes of intermittent reperfusion were observed and stable reperfusion was achieved in 32 patients. Arrhythmias occurring within five minutes before or after ST segment resolution and coronary recanalisation on arteriography were defined as reperfusion arrhythmias. Transient reperfusion arrhythmias were seen in six patients during 11 of the 68 episodes of reperfusion with ventricular fibrillation (VF) in two patients, ventricular tachycardia (VT) in two, idioventricular rhythm (IVR) in two, frequent ventricular ectopics (VEs) in three, and bradycardia in two. During persistent ST segment elevation before reperfusion in 32 patients, arrhythmias were observed in 21 with VF in three (in two during cardiac catheterisation), VT in 14 (8), IVR in 4 (2), VEs in 11 (7), AF in 5 (3) and bradycardia in 2(2). During the two hours after stable reperfusion, arrhythmias occurred in 12 patients, with VF in two, VT in seven, IVR in five, VEs in seven, AF in two and bradycardia in one.

Arrhythmias are commonly observed in patients during evolving acute myocardial infarction and thrombolysis, but specific reperfusion arrhythmias are not common.
Pre-hospital thrombolytic treatment with streptokinase for acute myocardial infarction in England, a cost effective treatment: comparison with secondary prevention using $\beta$ blockade

\section{R A Greenbaum \\ Harefield Hospital, Uxbridge}

Intravenous streptokinase achieves both reperfusion and improvement of prognosis in acute myocardial infarction. The purpose of this study was first to estimate the effect and cost if this policy were applied nationally and treatment was started before hospital admission. Secondly, this study compared the efficacy of this treatment with one year's secondary prevention treating survivors with $\beta$ blockers. In England during 1985 there were 99400 deaths attributed to acute myocardial infarction. Deaths in hospital were $26800 ; 72600$ died outside. Hospital admissions were 107000 . Thus total infarcts were at least 179600 . A previous study has shown that $\beta$ blockade started about the time of hospital discharge could at best provide a potential saving of 2480 lives in one year if all 80000 survivors could be treated. The cost would be about $£ 5028000$, an average of $£ 2030$ per life saved. The cost of treatment with streptokinase ( 1.5 million units) is approximately $£ 100$ per patient. If all infarcts were treated with streptokinase outside hospital and there was a $20 \%$ reduction in mortality there would be a saving of 19900 lives at a cost of $£ 17960000, £ 900$ per life saved. This is eight times the best improvement in lives saved that could be expected with one year's treatment with $\beta$ blockade. More realistically, if $30 \%$ of the annual number of infarcts (53900) were treated with streptokinase before hospital admission 5960 lives could be saved at a cost of $£ 5390000$.

For about the same cost, pre-hospital streptokinase treatment could save 2.5 times the number of lives as one year's secondary prevention with $\beta$ blockers.

Thrombolytic treatment in acute myocardial infarction: acute ischaemic events during long term follow up

A Timmis, R Henderson, A Pipilis, V Paul, E Sowton

Guy's Hospital, London

Although thrombolytic treatment is widely used in myocardial infarction, late coronary reocclusion may expose the patient to the risk of further ischaemic events. We have examined the incidence of acute 
ischaemic events in 44 patients with angiographically proved coronary recanalisation after myocardial infarction. Twenty six patients (group A) received early revascularisation (percutaneous transluminal coronary angioplasty $n=20$, coronary artery bypass graft $(n=6)$ at $17(5)$ days and 18 (group B) were treated conservatively. The treatment allocation was arbitrary and determined principally by the referring physicians. Nevertheless there were no significant differences between groups $\mathbf{A}$ and $\mathbf{B}$ in infarction location, number of diseased vessels-1.9(0.2) and $1 \cdot 6(0 \cdot 1)$, respectively-or duration of follow up16(2) and 13(3) months, respectively. There was a total of nine acute ischaemic events (death 2, myocardial infarction 4, unstable angina 3) affecting $20 \%$ of the patients during a follow up period of 15(2) months. Of the nine events, however, eight occurred in group $B$ compared with only one (unstable angina) in group $A(p<0.01)$.

The infarct patients included in this study all had proved coronary recanalisation and were therefore a potentially high risk group for further ischaemic events. The data, however, indicate that the incidence of events was not excessive in relation to the expected incidence in other survivors of myocardial infarction. Indeed, in patients treated with early revascularisation the incidence of acute ischaemic events during follow up was strikingly low.

\section{Early versus late recombinant tissue plasminogen activator (rt-PA) in acute myocardial infarction}

A J McNeill, S R Cunningham, D J Flannery, L M Burgess, E Tsoi, C M Wilson, N P S Campbell, M M Khan, G C Patterson, S W Webb, A A J Adgey Regional Medical Cardiology Centre, Royal Victoria Hospital, Belfast

Twenty nine consecutive patients ( 5 women, 24 men) mean age 58 years (range 37-74) with myocardial infarctions (MI) of $<4$ hours duration were randomised when first seen to receive either $100 \mathrm{mg}$ rt-PA over 90 minutes followed by $50 \mathrm{mg}$ over $3 \frac{1}{2}$ hours (group $A, n=14$ ) or to placebo (group $B$ $\mathrm{n}=15$ ). On admission, patients who had received placebo were given rt-PA and vice versa via an infusion running concurrently with the first infusion. On average rt-PA started 110 minutes after the $\mathrm{MI}$ in group $\mathrm{A}$ and 192 minutes in group $\mathrm{B}$. Cardiac catheterisation was performed at a mean of 10 days for group A patients and 11 days for group B. MI related vessel patency was assessable in 13 patients from each group. Of 13 in group A, 10 had TIMI grade 2 or 3 compared with 6 of 13 in group
B. Mean global ejection fraction (GEF) for group A was $49 \%$ and $44.5 \%$ for group B. For those with anterior MI (group A) mean GEF was $44 \%$; it was $45 \%$ for those in group B. For those with inferior MI (group A) mean GEF was $52 \%$ compared with $48 \%$ for those in group B. Regional wall movement in right anterior oblique $30^{\circ}$ view was assessed by the Leighton method. Patients in group A had a mean of 3.5 hypokinetic segments compared with 3.9 for group B. Mean percentage shortening of segments 2 and 3 in patients with anterior MI was $18 \%$ for group $\mathrm{A}$ and $14 \%$ for group B. Mean percentage shortening of segments 7 and 8 in patients from group A with inferior MI was $13 \%$ compared with $6.5 \%$ in patients from group $B$. There were no important bleeding complications. Five patients had reocclusions-one in group $\mathbf{A}$ and four in group $\mathbf{B}$. During their stay in hospital two patients (one from each group) died from cardiac disease.

Rt-PA is an effective thrombolytic agent that can safely be given outside hospital. Early administration suggests better reperfusion rates and improved left ventricular function but a larger number of patients are required to verify these results.

\section{Post infarction ventricular septal defect: value of colour flow mapping}

J Smyllie, K Dawkins, N Conway, G R Sutherland Wessex Cardiothoracic Unit, Southampton

Previous ultrasound studies of post infarction ventricular septal defects (PIVSD), restricted to duplex scanning have suggested important limitations inherent in the technique. Potentially colour flow mapping (CFM) should solve these problems by accurately confirming both defect site(s) and VSD jet direction, thus allowing more accurate noninvasive haemodynamic assessment. We report a prospective correlative study comparing both techniques in which 30 patients with PIVSD (22 new, eight recurrent) were studied both before and after operation. Duplex information was available in all 30 and CFM in 13. The defect was imaged by cross sectional echocardiography in only five studies (one inferior, three apical, one central PIVSD). Duplex scanning correctly showed a right ventricular flow disturbance diagnostic of PIVSD in 29 studies, but failed to locate the defect in 12 cases and provided ambiguous haemodynamic data in 19 . In contrast CFM consistently predicted defect site as well as jet width, direction, and number of discrete defects. Three differing CFM patterns diagnostic of (a) inferior, (b) apical, and (c) central PIVSD were defined. Evaluation of PIVSD flow velocity profiles showed 
a number of differing shunting patternspandiastolic shunting was a consistent feature with variable late diastolic shunt reversal. PIVSD jet velocity $(n=29)$ was found to be less accurate in right ventricular pressure estimation than tricuspid incompetence velocity. Both early $(n=2)$ and late $(\mathbf{n}=5)$ patch dehiscence and the development of further septal rupture $(n=2)$ were accurately predicted.

We conclude that colour flow mapping has significant advantages over non-CFM duplex scanning and is the ideal non-invasive technique for the identification and assessment of PIVSD both before and after operation.

Colour flow Doppler ultrasound in assessment of prosthetic valve function: an interim analysis

J B Chambers, G Jackson

Cardiac Unit, King's College Hospital, London

We have used colour flow Doppler ultrasound (CFD) to study 90 prostheses in 80 patients over a six month period. We used a Hewlett Packard system with threshold enhanced and variance maps, a 1.9 $\mathrm{MHz}$ continuous wave (CW) and $2.5 \mathrm{MHz}$ duplex transducers. The study was conducted as follows: cross sectional echơcàrdiography, CFD, pulsed Doppler, and CW. Normal function was defined clinically and by normal pressure half times and transvalve velocities, or both. Regurgitation was shown by a jet with multiple aliasing. It was considered paraprosthetic $(\mathbf{P} / \mathbf{P})$ if a thread of colour was seen outside the sewing ring and through the valve if the jet was seen within the orifice on at least two views. All normal mitral prostheses had mosaic aliasing and variance throughout diastole. Characteristic inflow patterns for Björk-Shiley $(n=9)$, Carpentier-Edwards $(n=13)$, and Starr-Edwards $(n=7)$ prostheses were shown. Mitral regurgitation was not detected. In normal aortic prostheses BjörkShiley valves $(n=14)$ had minor regurgitation through the valve in 12 , and Carpentier-Edwards valves $(n=11)$ had mosaic aliasing through the whole orifice and no regurgitation. In abnormal valves. clinically unsuspected regurgitation was found in six mitral and 11 aortic valves. Thirteen had small $\mathbf{P} / \mathbf{P}$ leaks and four had leaks through the valve. Three further valves had regurgitation on $C W$ but not on CFD. Catheterisation in five cases validated the site of regurgitation by CFD. Ten patients with regurgitation previously detected but not localised by conventional Doppler were reexamined by CFD with successful localisation in nine.
Our initial experience shows CFD to be useful for quick detection of small unsuspected leaks, localisation of leaks, and visualisation of flow direction. Very small leaks may not be detected.

Extent of myocardial uptake of indium-111 monoclonal antimyosin antibody and ECG location of acute myocardial infarction

D Jain, A Lahiri, U Rawal, J C W Crawley, S Bhattacharya, T Smith, G D Zanelli, E B Raftery Northwick Park Hospital and Clinical Research Centre, Harrow

The diagnostic role of indium-111 labelled monoclonal antimyosin antibody ( ${ }^{111}$ In-AM) antigen binding fragment (Centocor) was assessed in 50 consecutive patients with suspected acute myocardial infarction (AMI). Serial creatine kinase enzyme release, 12 lead ECG, and ${ }^{111}$ In-AM uptake were measured in all patients. ${ }^{111} \mathrm{In}-\mathrm{AM}(2 \mathrm{mCi})$ was injected intravenously within 48 hours of the onset of chest pain. Imaging was performed in multiple views at 24 and 48 hours after injection using a calibrated gamma camera. Strict quality control was maintained for labelling and imaging. The scans were read blind by two observers. Images were obtained in 46 patients ( $35 \mathrm{men}$ ); 32 had AMI diagnosed by at least two of three conventional criteria: chest pain, a rise in creatine kinase activity and ECG changes; 24 had transmural and eight non-transmural AMI; three had unstable angina (no AMI), and 11 had no evidence of AMI or ischaemia. All patients with AMI had positive myocardial uptake of ${ }^{111}$ In-AM. One patient with non-ischaemic pain had a diffuse uptake of ${ }^{111} \mathrm{In}-\mathrm{AM}$, the rest of the non-AMI group had negative scans (13 of 14). Total agreement of location of infarct between ECG and ${ }^{11}{ }^{1}$ In-AM was found in 9 of 32; ECG overestimation in four of 32 (only inferior); ECG underestimation in 16 of 32; and positive scan with negative ECG in three.

${ }^{111}$ In-AM is highly sensitive for detecting myocardial necrosis as determined by a rise in creatine kinase activity. The 12 lead ECG underestimates infarct size and location. ${ }^{111}$ In-AM produces easily interpretable images and may be valuable for determining prognosis and infarct size.

\section{Are multiple ventricular septal defects consistently predicted by colour flow mapping?}

G R Sutherland, B R Keeton

Wessex Cardiothoracic Unit, Southampton General Hospital 
Duplex scanning can accurately define both the type of ventricular septal defect (VSD) and associated haemodynamics in isolated VSDs; this may allow surgical referral on non-invasive grounds alone. One caveat to this approach has been the possibility of missing multiple VSDs. Colour flow mapping (CFM) has the potential to show the number and site of the VSDs, thus precluding preoperative angiography. To determine the accuracy of CFM diagnosis of VSDs, we have studied 31 patients with a broad range of such defects comparing CFM information with both angiographic and operative findings. Patients were analysed in two groups-group 1: 13 patients with equal ventricular peak systolic pressures and group 2: 18 patients with dissimilar ventricular pressure. In group 1 both imaging and CFM were unreliable in predicting the presence of multiple VSDs showing two or more discrete VSD jets in only eight of 13 patients. The combination of apical trabecular and single perimembranous defect was consistently predicted (all three patients) but multiple adjacent defects sited in perimembranous, muscular outlet, or muscular inlet septae were not accurately defined by either CFM or correlative left ventricular (LV) angiography. Where ventricular pressures were dissimilar (group 2) multiple defects were more accurately predicted (16 of 18 patients) but similar problem areas still existed. CFM was demonstrably better than LV angiography in this group. Multiple trabecular VSDs (not visualised by Duplex) were consistently predicted by CFM in both groups.

We conclude that CFM is a useful adjunct in the diagnosis of multiple VSDs, and, in our experience, is more accurate than $\mathrm{LV}$ angiography. It fails to be consistently accurate, however, in defined subsets of the multiple VSD population.

\section{Incidence and coincidence of prosthetic valve regurgitation assessed with colour flow imaging}

D Kambouroglou, Jayshree Joshi, G Karatasakis, P Nihoyannopoulos

Royal Postgraduate Medical School, Hammersmith Hospital, London

Colour Doppler echocardiography has been used to assess the degree of regurgitant lesions. in patients with prosthetic valves. We have observed several patients with clinically normal prosthetic valve function but with appreciable regurgitation on colour flow imaging. To assess the frequency of this finding we studied 176 patients with normally functioning aortic and mitral prostheses. In the aortic position,
38 patients had Starr-Edwards valves, 19 BjörkShiley, and 25 had bioprostheses. In the mitral position, 50 patients had Starr-Edwards valves, 22 Björk-Shiley, and 22 had bioprostheses. No patient was clinically in heart failure. Colour flow imaging showed the presence of aortic incompetence in $\mathbf{3 8}$ of $82(67 \%)$ patients of whom 19 of $38(50 \%)$ had StarrEdwards, 12 of $19(63 \%)$ Björk-Shiley and seven of $25(28 \%)$ had bioprostheses. Mitral incompetence was detected in 15 of $94(16 \%)$ patients of whom nine of $50(18 \%)$ had Starr-Edwards prostheses, one of 22 $(4 \%)$ Björk-Shiley, and five of $22(23 \%)$ had bioprostheses. In only 10 patients was there clinical evidence of an aortic $(n=9)$ or mitral $(n=2)$ regurgitant murmur, on the basis of a well functioning prosthesis.

We conclude that mild prosthetic valvar regurgitation is found in one of three patients with normal prosthetic valves. Patients with aortic prostheses show a higher frequency of regurgitation than those with mitral prostheses $(p<0.05)$.

\section{Cine magnetic resonance imaging of valvar heart disease}

S R Underwood, R Mohiaddin, D N Firmin, R H Klipstein, H Bogren, R S O Rees, D B Longmore

Magnetic Resonance Unit, National Heart and Chest Hospitals, London

Regurgitant fraction can be calculated in patients with valvar disease from magnetic resonance stroke volume measurements, provided only a single valve is affected. A cine field echo sequence is more flexible as turbulent flow leads to loss of signal and to a qualitative assessment of abnormal flow patterns. We have studied 20 normal subjects and 30 patients with stenosis or regurgitation, or both, of the aortic, mitral, and tricuspid valves. Turbulence was graded proximal and distal to the valves according to its area. Regurgitant jets were not seen in the normal subjects, but there was some systolic turbulence in the aorta and diastolic turbulence in the left ventricle during rapid mitral filling. In the patients with regurgitation of a single valve, there was a good correlation between the regurgitant fraction and the grade of turbulence $(r=0.85)$, and there were significant differences of mean regurgitant fractions in each grade $(p<0.01)$. The grade of turbulence agreed with the angiographic grade of regurgitation in 16 patients, but in seven it differed by one grade. Correlation was poor between the grade of turbulence in the ascending aorta and left ventricle and the pressure gradient across the aortic and mitral valves 
because turbulence occurred distal to abnormal but not to stenosed valves. The cine acquisition also showed details of valve disease: thickening and restricted motion of stenosed valves was apparent, and left atrial thrombus was more clearly seen than in the spin echo images.

A cine field echo sequence with spin echo volume measurements provides a good assessment of regurgitant aortic, mitral, and tricuspid valves, but turbulence distal to abnormal valves occurs irrespective of the degree of stenosis.

\section{Risk of electromagnetic interference with pacemaker function in the aircraft environment}

W D Toff, A G McC Deller, R A Hobbs, D J Robb, M Joy, A J Camm

UK Civil Aviation Authority, London

The electromagnetic environment on board aircraft presents a potential hazard to pacemaker users, but the level of risk has not been defined. Electromagnetic field strengths were measured on 10 aircraft during radio and radar operation. All had very high frequency (VHF) radio, four also had high frequency (HF) radio and nine had radar. Ten different unipolar pacemakers were exposed to similar fields at strengths up to $30 \mathrm{~V} / \mathrm{m}$ in a shielded chamber and the minimum field strengths for interference (abnormal pacing or reversion to interference mode) and failure (cessation of pacing for 30 seconds) were determined. The effect of modulation at $1 \mathrm{kHz}$ with pulsing at $2 \mathrm{~Hz}$ was also examined in $\mathrm{HF}$ fields. In the aircraft, maximum field strengths ranged from 0.2-35.0, mean (SD.) $6.55(10.95), \mathrm{V} / \mathrm{m}$ for VHF; $0 \cdot 2-1 \cdot 12$, mean $0.54(0.38), \mathrm{V} / \mathrm{m}$ for $\mathrm{HF}$; and $1 \cdot 12-18 \cdot 0$, mean $7 \cdot 23(5 \cdot 86), \mathrm{V} / \mathrm{m}$ for microwave. In the shielded chamber, all pacemakers were susceptible to interference and failed at some stage, often abruptly without prior reversion to interference mode. Minimum field strengths for interference ranged from $3-13$, mean $5 \cdot 7(2 \cdot 83), \mathrm{V} / \mathrm{m}$ for VHF; $1.5-7 \cdot 5$, mean $4.27(2 \cdot 24), \mathrm{V} / \mathrm{m}$ for HF; and $0.25-0.9$, mean $0.5(0.18), \mathrm{V} / \mathrm{m}$ for pulsed, modulated HF. Minimum field strengths for failure ranged from 3-22.5, mean $11 \cdot 2(6 \cdot 2), \mathrm{V} / \mathrm{m}$ for VHF; 6-10, mean $7 \cdot 7(1 \cdot 33), \mathrm{V} / \mathrm{m}$ for continuous wave $\mathrm{HF}$; and $0.55-2.9$, mean $1.51(0.87), \mathrm{V} / \mathrm{m}$ for pulsed, modulated HF. In microwave fields, interference occurred in only one unit (at $16 \mathrm{~V} / \mathrm{m}$ ) and there were no failures.

It is concluded that pacemakers are susceptible to interference in electromagnetic fields comparable to those found on aircraft. The extent to which implantation offers shielding and reduces the risk to patients requires evaluation.

\section{Refinement of risk stratification after myocardial infarction by programmed electrical stimulation of the right ventricle}

M A de Belder, J D Skehan, C W Pumphrey, $S$ J W Evans, P G Mills, $M$ T Rothman Cardiac Department, The London Hospital, London

A sub-maximal exercise test (SMET) following myocardial infarction (MI) selects a group at risk of death in the subsequent year. To evaluate the role of repetitive ventricular responses (RVRs) to programmed electrical stimulation (PES) of the right ventricle in refining risk stratification, we studied 262 consecutive stable survivors of $\mathrm{MI}$, all of whom undertook an early SMET: those with a positive test underwent cardiac catheterisation and PES. RVRs were sought with one or two ventricular extra stimuli in sinus rhythm or paced at 600 and $500 \mathrm{~ms}$ cycle lengths. Patients with a positive SMET were 13 times more likely to die in the first year than those with a negative test. In those patients with a positive SMET there were no responses to PES in $15 \%$, up to four in $65 \%$, between five and 22 in $14 \%$, and $5 \%$ developed sustained ventricular tachycardia (VT) $\geqslant 10 \mathrm{~s}$. No deaths occurred with the investigation Sustained VT was independent of site and type of infarct, extent of coronary disease and left ventricular dysfunction, Lown grading of ambulatory ECGs, as well as clinical and SMET induced arrhythmias. Multivariate analysis showed that induced sustained VT was the most powerful predictor of death in the first year; patients with this response were 10 times more likely to die than those with no response.

Programmed electrical stimulation of the right ventricle for patients with a positive SMET thus strongly refines risk stratification.

First derivative of right ventricular pressure as a sensor for an implantable rate responsive VVI pacemaker

R Sutton, A Sharma, Ann Ingram, J Camm, $F$ Lindemans, $T$ Bennett

Westminster and St George's Hospital, London, University Hospital, London, Ontario, Canada, and Medtronic Inc, USA

After a preliminary series of pressure sensing nonrate responsive pacemakers, four patients mean age 
69 (range 43-87) with complete atrioventricular (AV) block have now had units implanted that offer them a rate response based on changes in the first derivative of right ventricular pressure. The system (Medtronic US) consists of a special unipolar lead with pressure transducer set $3.5 \mathrm{~cm}$ back from the stimulation electrode. The pacemaker has two connection ports and is equivalent in size and weight to the DDD unit. Implantation technique was straightforward and in three patients qualitative pressure and first derivative of pressure wave forms were comparable between those telemetered by the pacemaker and a fluid filled manometer. Follow up (mean two months) has assessed rate responsive behaviour by exercise stress testing, Holter monitoring, and tilting. Two patients have shown occasional periods of over sensing of $T$ waves, otherwise no complications have been encountered. Resting heart rate before exercise was a mean of 66 paces per minute (ppm) with rapid acceleration in the first minute of exercise and the mean maximum heart rate achieved was $114 \mathrm{ppm}$. The mean rate variability was 48 between rest and peak exercise, rate fall on recovery was physiological over five minutes. Mean heart rate change on Holter monitoring was from 53 to $90 \mathrm{ppm}$. Mean heart rate on tilt increased from 64 to $71 \mathrm{ppm}$ at $60^{\circ}$ head up.

In conclusion, sensing of the first derivative of right ventricular pressure for rate responsive ventricular pacing proves in practice to be physiological.

\section{Is the optimal atrioventricular delay the same at rest and during exercise? An assessment in patients with dual chamber pacemakers}

\section{Mehta, Susan Gilmour, C P Lau, N Mehta, D E Ward, A J Camm \\ St George's Hospital Medical School, London}

Cardiac output is a product of heart rate and stroke volume. In a patient with a dual chamber pacemaker the pacing rate is dependent on the atrial rate, and the stroke volume depends in part on appropriate timing of atrial systole. We investigated the optimal atrioventricular (AV) interval during exercise using continuous wave Doppler. Cardiac output was calculated as the product of Doppler measured stroke distance at the left ventricular outflow (using Quinton Exerdop equipment), aortic root area ( $M$ mode echocardiogram), and pacing rate. Nine patients (average age 53, range 22-71 years) with persistent complete heart block, normal left ventricular function, and implanted dual chamber pacemakers, were studied. Pacemakers were programmed to a basic pacing rate of $70 \mathrm{bpm}$. At rest cardiac output was measured with AV intervals of $75,100,150$, and $200 \mathrm{~ms}$. Cardiac output was then measured at the peak of four submaximal exercise tests (stage I Bruce protocol), at each of the above AV intervals. At rest, cardiac output was maximum with an AV delay of $150 \mathrm{~ms}$ mean (SD) $(5.81(1.5) 1 / \mathrm{min})$, and it was significantly lower at AV intervals of $75(5 \cdot 2(1 \cdot 3)$ $1 / \mathrm{min}, \quad p<0.01)$ and $200 \mathrm{~ms}(5.51(1.5) 1 / \mathrm{min}$, $\mathrm{p}<0.01)$. On exercise, maximum cardiac output was seen with an AV delay of $75 \mathrm{~ms}(8.261 / \mathrm{min})$ which was significantly higher than that at $\mathrm{AV}$ intervals of $150(7.46(0.9) 1 / \mathrm{min}, \mathrm{p}<0.05)$ and $200 \mathrm{~ms}$ $(7.85(1.1) 1 / \mathrm{min}, \mathrm{p}<0.05)$. The changes in heart rate on exercise at all $\mathrm{AV}$ intervals were similar.

We conclude that the optimal atrioventricular delay is shorter during exercise than at rest. Rate modulation of the $\mathrm{AV}$ interval will improve the haemodynamic response in patients fitted with dual chamber pacemakers.

\section{Atrial natriuretic peptide in different modes of cardiac pacing}

P Vardas, C M Travill, T D M Williams, Ann Ingram, S L Lightman, $R$ Sutton Westminster Hospital, London

Plasma atrial natriuretic peptide concentrations are raised in congestive cardiac and renal failure, and in paroxysms of supraventricular tachycardia. We investigated 32 patients, 20 men, mean age 69 years (range 38-86) with complete heart block but no heart or renal failure. Six patients with complete atrioventricular block had atrial natriuretic peptide concentrations (mean (SEM) pmol/l) of $141(28)$ unpaced, 139 (30) with ventricular pacing (VVI) for 30 days and $53(7)$ with atrioventricular pacing (DDD) for 30 days. Thirteen patients paced long term VVI (mean 43 months) had atrial natriuretic peptide concentrations of 112 (13). Thirteen patients paced long term DDD (mean 40 months) had peptide concentrations of $43(7)$. Thirteen age and sex matched controls had atrial natriuretic peptide concentrations of 58(7). There was a significant difference between atrial natriuretic peptide in unpaced, VVI paced for $\mathbf{3 0}$ days, and long term VVI paced complete heart block at the $5 \%$ level compared with controls. There was no significant difference between atrial natriuretic peptide after both 30 days or long term DDD pacing with controls. Four patients had similar resting concentrations of atrial natriuretic peptide when paced VVI or ventricular rate responsive pacing (VVIR): 133(34) and 134(39), respectively. During exercise atrial natriuretic peptide concentrations were lower in 
VVIR mode than VVI, 265(110) and 334(115), respectively, at identical exercise levels that were equivalent to peak exercise in VVI.

These results corroborate the physiological nature of DDD pacing compared with VVI and suggest that VVIR pacing offers benefit on exercise over VVI.

\section{Comparative assessment of rate responsive pacemakers}

S C Webb, L M Lewis, Jayne Morris-Thurgood, A Maseri

Royal Postgraduate Medical School, Hammersmith Hospital, London

The majority of studies designed to assess the performance of rate responsive pacemakers (RRPs) have been based on bicycle or treadmill exercise testing. This disregards the fact that few patients voluntarily undertake sustained exercise under progressively increasing workloads during their normal daily life. We have therefore used ECG telemetry to monitor changes in heart rate during walking, climbing stairs, and a range of standardised domestic tasks in 20 patients with RRPs (mean age 58 years). These pacemakers sensed activity $(n=12)$, respiration $(n=5)$, or QT interval $(n=3)$. Pacemaker responses were compared with the normal pattern of changes in heart rate recorded from 20 healthy volunteers (mean age 61 years) undergoing the same activities. All RRPs could be programmed to produce a close approximation to the normal heart rate response during walking, but there were considerable variations in their ability to meet the requirements of short periods of exertion. For example, mean values for the peak increase in heart rate $(R)$ during rapid stair climbing and the time taken (T) to reach this peak were as follows: controls- $R=58 \mathrm{bpm}, \mathrm{T}=43$ seconds; activity sensing- $R=39 \mathrm{bpm}, T=47 \mathrm{sec}-$ onds; respiratory sensing- $R=13 \mathrm{bpm}, T=80 \mathrm{sec}-$ onds; $Q T$ sensing- $\mathrm{R}=31 \mathrm{bpm}, \mathrm{T}=145$ seconds.

The delayed responses found with respiratory and QT sensing pacemakers led to peak increases in pacing rate occurring at up to 90 seconds after the cessation of some forms of exercise. Such response patterns may give rise to symptomatic postexertional tachycardia.

\section{Coronary artery stenting after balloon angioplasty}

A F Rickards, P Urban, $M$ Bertrand, P Serruys, U Sigwart, J Puel

National Heart Hospital, London, and the Coronary Artery Stent Implantation Study Group
Restenosis, occurring in about one third of cases after coronary balloon angioplasty, is the major long term limitation of the technique. In an attempt to overcome this, a new flexible, self expanding stainless steel endoprosthesis has been developed and initial results have been encouraging. The stent can be inserted percutaneously using a standard angioplasty guide catheter. Immediately after successful balloon angioplasty, we have implanted four such devices in the left anterior descending coronary artery (LAD) of four male patients with either recurrent stenosis $(n=3)$ or new disease. All had good left ventricular function without previous $Q$ wave infarction in the LAD territory. None had unstable angina, but all had exertional symptoms and a positive stress test. A good immediate angiographic result was obtained in all cases with no appreciable residual stenosis.

No early complications occurred as a result of stent implant and no fixed or transient postoperative ECG changes occurred. There have been no complications during the follow up period of one to four months. Meticulous anticoagulation was maintained continuously during and after implantation together with administration of aspirin and calcium channel blockers. In addition to describing our own experience, an overview of the data obtained by the Coronary Artery Stent Implantation Study group will be presented.

\section{Angioplasty for occluded and disconnected coronary arteries}

A Perakis, R Henderson, A Timmis, E Sowton Guy's Hospital, London

Indications for percutaneous transluminal coronary angioplasty (PTCA) are expanding and have come to include totally occluded vessels. We have undertaken single vessel PTCA in 29 patients with total coronary occlusion (group 1). A further 55 procedures were performed in patients with coronary disconnection (group 2), with no visible connection between proximal and distal arterial segments despite delayed antegrade filling. In both groups $75 \%$ of patients had only single vessel disease, and 0 $45 \%$ had had infarction in the territory of the PTCA vessel. Despite this all patients complained of troublesome angina, which was severe (grade III or IV) 0 in $79 \%$ of group 1 and $87 \%$ of group 2 . PTCA was angiographically successful in $55 \%$ of group 1 and $\stackrel{\mathbb{\Omega}}{\mathbb{D}}$ $80 \%$ of group $2(p<0.05)$; success was not $\stackrel{\mathbb{Q}}{\varrho}$ influenced by the estimated duration of arterial $\bar{O}$ occlusion. There were no deaths in hospital in either 8 group. Indeed, group 1 had no major complications, $\frac{8}{0}$ 
but three patients in group 2 required emergency operations, and two had myocardial infarctions in hospital. Successful PTCA led to a sustained symptomatic improvement in most patients with follow up ranging up to four years.

These data indicate that patients with angina associated with coronary occlusion or disconnection respond well to PTCA. An anterograde connection between proximal and distal arterial segments significantly improves the PTCA success rate. Although total occlusion has a lower success rate the procedure is safe and symptoms will often improve if the vessel is reopened.

\section{Effect of intracoronary nitrate and nifedipine on regional myocardial blood flow and metabolism during coronary angioplasty}

\author{
R A Perry, A Seth, S C H Smith, Faith Bailey, \\ M F Shiu \\ University Department of Cardiovascular Medicine, \\ Queen Elizabeth Hospital, Birmingham
}

Intracoronary drugs are often given during coronary angioplasty (PTCA) as prophylaxis against coronary spasm. To investigate any cardioprotective effect we measured great cardiac vein flow (GCVF) by thermodilution, regional myocardial oxygen consumption $\left(\mathrm{MVO}_{2}\right)$, lactate extraction ratio (LER) and intra-coronary ECG changes, during four consecutive balloon inflations each of 60 seconds in 12 men undergoing dilatation of the left anterior descending coronary artery. Inflation 2 was used for control readings. Inflations 3 and 4 were undertaken 60 seconds after intra-coronary injection of $200 \mu \mathrm{g}$ of nifedipine and $200 \mu \mathrm{g}$ glyceryl trinitrate, respectively. Baseline readings were taken before and between inflations. During control inflation mean GCVF (ml/min) fell significantly (102 (32)-82(30), $\mathrm{p}<0.02) ; \quad \mathrm{MVO}_{2} \quad(\mathrm{ml} / \mathrm{min})$ was reduced $(12 \cdot 3(4 \cdot 2)-10 \cdot 1(2 \cdot 5), \mathrm{p}<0.05)$ and there was net lactate production (64(70)-12(92), $p<0.05)$. After intracoronary nifedipine, during inflation 3 there was an increase in GCVF (94(22)-125(52), $\mathrm{p}<0.02)$, no significant fall in $\mathrm{MVO}_{2}$ $(11.3(2.9)-10.9(3.4))$ and no net lactate production (29(58)-41 (38)). There was no change over control values in GCVF (98(34)-74(41), p < 0.02) or $\mathrm{MVO}_{2}(11.8(4 \cdot 3)-9.5(2.9), \mathrm{p}<0.05)$ after intracoronary glyceryl trinitrate but the fall in LER was less pronounced (13(75)-7(80), NS). Continuous monitoring of the intracoronary ECG showed a reduction of ST segment rise during balloon inflation preceded by nifedipine and by glyceryl trinitrate $(4.5(2) \mathrm{mm}$ and 5.5 (3) $\mathrm{mm}$ compared with control of $7.0 \mathrm{~mm}, \mathrm{p}<0.005$ and $\mathrm{p}<0.05$, respectively).

In conclusion, balloon coronary occlusion for 60 seconds resulted in significant falls in regional myocardial blood flow, oxygen consumption, and lactate production. These changes were completely abolished by prior administration of intracoronary nifedipine. Intracoronary nitrates in this context do not affect coronary flow but may attenuate regional ischaemia to a lesser extent than nifedipine.

\section{Use of pulsed Nd-YAG laser energy for laser assisted balloon angioplasty in man}

T J Bowker, F W Cross, S G Bown, Penny Shaw, M Adiseshiah, J A P Marston, A F Rickards

National Heart and University College Hospitals, London

The risk of vessel perforation in laser assisted balloon angioplasty (with conventional continuous wave lasers) is reduced by the use of modified fibre tips, allowing lesions to be crossed which cannot be crossed by conventional means alone. Higher peak power pulsed laser energy causes less subjacent tissue damage than does continuous wave laser energy and may further reduce the risk of perforation. To assess the clinical safety and efficacy of $100 \mu$ s pulsed $\mathrm{Nd}-\mathrm{YAG}$ laser energy $(0.5 \mathrm{~J}$ pulses at $10 \mathrm{~Hz}$, and peak power density of $10 \mathrm{MW} / \mathrm{cm}^{2}$ ) delivered via a $600 \mu \mathrm{m}$ core diameter fibre with a $2 \mathrm{~mm}$ diameter bullet-shaped sapphire tip, percutaneous laser assisted balloon angioplasty was performed on superficial femoral artery occlusions which could not be crossed with a guidewire, in 5 patients (mean age $74 \cdot 2(4 \cdot 8)$ years), all of whom had rest pain and four of whom faced amputation. All five occlusions were recanalised successfully without perforation using laser energy (mean total dose per occlusion$129(34) \mathrm{J}$ ), and permitting subsequent balloon dilatation. The mean pretreatment Doppler ankle pressure index of $0.27(0 \cdot 12)$ increased to $0.5(0 \cdot 15)$ after treatment. In the two vessels with poor run off reocclusion occurred within 48 hours requiring below knee amputation. The other three patients have patent vessels, with abolition of rest pain and limb salvage.

Peripheral laser assisted balloon angioplasty with pulsed Nd-YAG laser energy and sapphire tipped fibres is safe and allows angioplasty to be performed when the balloon alone is unsuccessful. 
Coronary angioplasty as a treatment for patients with angina pectoris after a non- $Q$ wave myocardial infarction

K J Beatt, H Suryapranata, P J de Feyter, J Verrostte, $M$ van den Brand, P G Hugenholtz

Thoraxcenter, Rotterdam, The Netherlands

More aggressive treatment has been suggested for patients who sustain a non- $Q$ wave myocardial infarction because of the subsequent unstable angina, recurrent myocardial infarction, and high mortality are common compared with patients with $Q$ wave myocardial infarction. We therefore analysed the outcome of 116 patients who had coronary angioplasty (PTCA) after a non- $Q$ wave myocardial infarction between 1981 and 1986 . All patients were symptomatic, $47 \%$ having unstable angina before angioplasty, which was performed a median of 31 days after the infarction. Nineteen per cent of patients had a totally occluded vessel at the time of attempted PTCA and the primary success rate (less than $50 \%$ stenosis) of the procedure was $87 \%$. A mean clinical follow up of 15 (range 3-59) months was obtained in all patients. Complications related to the angioplasty were emergency surgery in seven $(6 \%)$ patients, and myocardial infarction in five $(4 \%)$. Late recurrent myocardial infarction occurred in four $(3 \%)$ patients and further revascularisation procedures were necessary in $19(16 \%)$ patients who had a further PTCA, and five ( $4 \%$ ) patients who had late coronary artery surgery. One patient died following operation after a failed PTCA. After a successful PTCA and if necessary a further PTCA, 73\% of patients were asymptomatic and on no antianginal treatment at follow up.

In this selected group of patients with a non-Q wave myocardial infarction the high initial success rate, low incidence of subsequent death and recurrent myocardial infarction, together with sustained symptomatic benefit, suggest that PTCA is an effective treatment.

Predictors of restenosis after percutaneous transluminal coronary angioplasty

R Henderson, A Timmis, $M$ Hughes, A Pipilis, E Sowton

Guy's Hospital, London

The long term efficacy of percutaneous transluminal coronary angioplasty (PTCA) is limited by early restenosis which affects 20 to $30 \%$ of patients. There is therefore an important need to identify risk factors for restenosis. We have analysed 20 baseline variables in a cohort of 328 patients after successful PTCA followed up for at least six months. Clinical relapse defined by symptomatic or exercise test criteria occurred in 97 patients, all of whom underwent repeat arteriography. In total, arteriographic follow up was available in 126 patients and coronary restenosis of at least $50 \%$ luminal diameter was shown in 70 patients $(21 \%) \cdot \chi^{2}$ testing identified emergency PTCA, residual stenosis, grade of angina, male sex, and positive predischarge stress test as significant $(p<0.05)$ univariate predictors of restenosis. Multivariate analysis was performed using stepwise logistic regression and restenosis risk ratios were calculated for each predictor variable (values in parentheses). Emergency PTCA (3.7), male sex (3.2), residual stenosis $(2 \cdot 4$ for $30 \%$ stenosis), and positive predischarge stress test $(2 \cdot 1)$ were all retained as significant $(p<0.05)$ independent predictors of restenosis.

These data indicate that clinical relapse after successful PTCA is not always associated with coronary restenosis. Patients at greatest risk of restenosis are men undergoing emergency PTCA, particularly when the residual stenosis remains appreciable $(30-50 \%)$. A positive predischarge stress test provides additional independently predictive data.

\section{Sustained long term improvement in left ventricular function after successful coronary angioplasty}

R A Perry, A Singh, Jane Flint, A Seth, R G Murray, $M$ F Shiu

University Department of Cardiovascular Medicine, Queen Elizabeth Hospital, Birmingham

Data on long term clinical efficacy of successful coronary angioplasty (PTCA) is now available. In order to evaluate the long term benefits in left ventricular (LV) function, 49 patients who had had rest and exercise $\mathrm{Tc}^{99 \mathrm{~m}}$ gated blood pool images before and six weeks after successful PTCA were studied at a mean interval of 15 months (range 9-24) after the procedure. None of the patients had clinical evidence of restenosis; 15 (group A) had post infarction angina whereas the remaining 34 (group B) had no previous infarction. In both groups before PTCA there was a significant fall in ejection fraction on exercise more pronounced in group A (A 48\% (10)-40\% (16), p < 0.001; B 58\% (10)-53(13), p < 0.005). This change was paralleled by a worsening wall motion score (A 2.3(1.9)-3.3(2.4), p< 0.01; B $0.6(0.4)-1.6(1.2), \mathrm{p}<0.001)$. Six weeks after the 
procedure there was no significant change in resting ejection fraction but there was a significant increase on exercise, particularly in group A (A 53\% (13), $\mathrm{p}<0.001 ; \mathrm{B} 62 \%(11), \mathrm{p}<0.001)$ with a concomitant improvement in exercise wall motion score (A $1.8(1 \cdot 1), \mathrm{p}<0.005 ; \mathrm{B} 0.4(0.6), \mathrm{p}<0.001)$. This improvement in exercise ejection fraction and wall motion was maintained at later follow up with no significant deterioration in either measurement and a clearly sustained significant improvement over prePTCA values for ejection fraction (A $51 \%$ (10), p < $0.005 ; \mathrm{B} 60 \%(10), \mathrm{p}<0.002)$ and wall motion (A $1 \cdot 3(0 \cdot 8), \mathrm{p}<0.001 ; \mathrm{B} 0 \cdot 2(0 \cdot 2), \mathrm{p}<0.0001)$.

Thus the immediate improvement in left ventricular function after successful PTCA is maintained in patients with and without previous myocardial infarct for up to two years.

\section{Coronary angioplasty in unstable angina: higher success rate and lower complications than in stable angina}

D Gilligan, P Gueret, B Maurer, M Walsh, P Crean, G Gearty

Royal City of Dublin Hospital, Dublin, Ireland

Unstable angina is associated with plaque rupture, coronary artery spasm, and intravascular clot formation, suggesting that coronary angioplasty may not be the ideal form of treatment. We therefore examined the success and complication rates of percutaneous transluminal coronary angioplasty in unstable angina compared with stable angina. Two hundred patients (250 lesions) underwent coronary angioplasty from November 1982 to February 1987. The indication for angioplasty was unstable angina in $118(59 \%)$ and stable angina in $69(35 \%)$ (others included acute infarction and after streptokinase reperfusion). The mean age was 53 years, 162 were men and 38 women, and $112(56 \%)$ had single vessel disease. The equipment, techniques, and drugs used were similar in stable and unstable angina. Primary success (at least $20 \%$ reduction in luminal stenosis without major complications) was achieved in 146 cases $(73 \%)$ and 192 lesions $(77 \%)$. Luminal stenosis was reduced from a mean of $87(11) \%$ to $27(15) \%$. In patients with unstable angina the success rate $(81 \%)$ was significantly higher than in those with stable angina $(57 \%) p<0.01$. Success rates were not significantly different between men and women, $73 \%$ and $71 \%$, respectively. Similar success rates were achieved in each of the main coronary arteries: left anterior descending 132 of $176(75 \%)$, right coronary 13 of $18(72 \%)$, and circumflex seven of 12 $(58 \%)$, NS.
Major complications (mortality, emergency coronary artery bypass grafting, or infarction) occurred in $12 \%$ of the whole group. In patients with stable angina major complications were significantly greater than in those with unstable angina, $22 \%$ versus $7.6 \%, \mathrm{p}<0.01$.

\section{Systolic and diastolic blood pressures in prediction of heart attacks and stroke}

A G Shaper, A N Phillips, S J Pocock, Mary Walker Royal Free Hospital School of Medicine, London

The relative importance of systolic and diastolic blood pressures in predicting risk and likely benefit from treatment remains controversial. The British Regional Heart Study provides data on $\mathbf{7 7 3 5}$ middle aged men followed for cardiovascular events for six years; 336 of the men have had an MI, fatal or nonfatal, and 71 men have had strokes. Systolic and diastolic blood pressures are strongly correlated, $r=0 \cdot 70$, but there is considerable discordance in the association. Their independent and combined ability to predict major cardiovascular events has therefore been examined. If diastolic blood pressure (DBP) is used as the basic measurement, an increment in systolic blood pressure (SBP) above the average figure associated with that DBP (for that age-group) increases the risk of a heart attack. Using SBP, a similar phenomenon is observed for increments in DBP, but the effect is less pronounced.

Our findings suggest that DBP should be used as the basic measure together with assessment of the systolic increment as a more precise guide to risk of ischaemic heart disease. For stroke, SBP appears to be paramount and increments in DBP above the average contribute no further risk. There are considerable implications in these observations for the design of clinical trials and for the management of raised blood pressure.

Variation in general practitioner referral for assessment of patients with chest pain: impact on need for coronary artery bypass grafting

M Joy, Charlotte Keywood, P M J Bennett, Kit Nicholson

St Peter's Hospital, Chertsey

The need for invasive cardiological assessment and revascularisation procedures is related to the prevalence of coronary artery disease. It is also related to the resources available and to the perception by patients and their general practitioners (GPs) of the 
possible benefits. We have previously noted a wide variation in the numbers of patients referred by individual GPs in north west Surrey for investigation of chest pain. This study sought to elucidate this phenomenon. Between 1982-6, 441 patients were referred for coronary angiography, 316 subsequently undergoing CABG (219) or PTCA (26). These patients originated from 89 principals in general practice, of whom 13 were excluded on the grounds of death, retirement, recent appointment, or small intradistrict practice ( $<1000$ patients). The GPs were ranked by total number of patients undergoing coronary angiography followed by CABG or PTCA, and by number per 1000 patients on the practice list. There was a mean of 4.32 patients per GP, referred by the district cardiological unit for coronary angiography originating from the top decile of GPs over the five year period, and of 0.56 patients from the bottom decile. The distribution represented an exponential decline. The figures for the uptake of surgery were 3.26 patients $/ 1000$ practice list five years, and 0.38 patients/ 1000 practice list/five years, respectively. If the referral rate of the district was that of the top 50th (rather than the 10th) centile of GPs, the need for the procedures was increased by $35 \%$ to about $380 \mathrm{CABG}$ per million population per annum (490 corrected for the low SMR in ICD 410-414). This difference cannot be accounted for in terms of GP years qualified (23.3 years and 20.5 years, respectively, $p=0 \cdot 196$, NS), nor was there detectable difference in the population structure between practices. Nevertheless a questionnaire seeking attitudes to referral disclosed that chest pain is a common symptom in general practice.

Current estimates of the national need for revascularisation procedures may seriously understate the true need, which is dependent on the awareness of both patients and their primary health care physicians.

Need for cardiac catheterisation and coronary artery surgery: experience of a district in the north west region

I Hussein, A J Behrana, N Naqvi

Department of Cardiology, Wigan Infirmary, Wigan

A six year study (1981-86) was undertaken of the experience of the Wigan district in the north west region of the need for invasive cardiological procedures. A similar study has been reported to the Society from Surrey. The catchment population is 310000 with a larger than expected number of people in social classes IV and V and a standardised mortality ratio for ischaemic heart disease of 1:28.
The district has a cardiologist with sessions for cardiac catheterisation at the Regional Cardiothoracic Centre, easy access to invasive cardiac procedures, a fully equipped non-invasive cardiac laboratory, and local follow up of cardiological and cardiac surgical patients by the cardiologist, and the regional cardiac surgeon. A total of 579 patients was referred for investigation of coronary artery disease, and 222 patients for valvar heart disease. There was a greater than fourfold increase in the number of patients investigated for coronary artery disease (116 procedures/million population/year in 1981 compared with 490 procedures/million population/year in 1986. There was little change, however, in the number of valvar heart disease referrals yearly, 120 procedures/million population/year being the average, much higher than the national average. Adjusting the Surrey figures to our district we estimate that we need 594 operations/million population/year for coronary artery disease, but in 1986 our figures were 343 operations/million population.

The reasons for this deficiency may well be lack of staff, (one cardiologist for a population of over 300000 ), an inadequate number of referrals from general practitioners and physicians, poor finance, and public ignorance of modern methods of management of coronary artery disease.

\section{Tentative localisation of the gene responsible for hypertrophic cardiomyopathy}

J Burn, C P Bennett, S Hunter, W J McKenna Department of Human Genetics, Freeman Hospital, Newcastle upon Tyne, and Hammersmith Hospital, London

Hypertrophic cardiomyopathy is a major cause of a sudden cardiac death in the young. Our regional clinical study has shown a minimum prevalence of $3 \cdot 2 / 100000$. Echocardiography has shown a large number of asymptomatic relatives such that the pedigree structure is compatible with a monogenic disorder inherited as an autosomal dominant with variable expression and occasional non-penetrance. The rapid recent advances in recombinant DNA technology make gene localisation feasible either by the candidate gene approach or by linkage mapping. We have assembled 10 families in whom the latter approach may be useful. We explain the techniques and request the cooperation of colleagues in order to identify further informative pedigrees. Preliminary studies have shown that the short arm of chromosome 6 is a likely site; reanalysis of seven published pedigrees, together with data from four of our own, 
has given a lod score of 3.04 at $12 \mathrm{~cm}$ from the major histocompatibility locus. This observation represents odds of 1000 to 1 in favour of the gene being close to the HLA locus.

Even using only affected cases the lod score of 1.7 is suggestive of linkage. If confirmed by DNA studies gene localisation will become feasible.

\section{Effect of serial tournament squash games on some cardiovascular and metabolic measurements}

T Lynch, M Kinirons, D O'Callaghan, Suad Ismail, M Ohman, J H Horgan

Department of Cardiology, St Laurence's Hospital, Dublin, Ireland

Metabolic changes have been proposed as a possible cause of sudden death occurring during and after playing squash. Such changes have been shown in relation to one match. There have been no studies, however, evaluating the cumulative metabolic response to repetitive tournament squash playing. We therefore compared the cardiovascular and metabolic responses to exercise, in repeated (three games) competitive squash, over a 48 hour period, in 10 men aged $>45$ years. Subjects were studied at rest before exercise, on completion of exercise, and five and 30 minutes later. The following were measured on each occasion: heart rate and rhythm, and plasma concentrations of potassium, magnesium, lactate, and free fatty acids. Aerobic and anaerobic fitness were assessed by exercise testing on a treadmill $\left(\mathrm{V}_{02}\right.$ $\max )$ and by ergometer. Statistical analysis was performed by Student's paired $t$ test. Repeated squash was associated with a similar fall in mean (SEM) plasma potassium $(3.84(0.1)$ to $3.65(0.1)$ and 4.05 $(0 \cdot 1)$ to $3.76(0 \cdot 1) \mathrm{mmol} / \mathrm{l}$, respectively, higher mean plasma lactate $(68.33(12.54)$ and $31.7(8.7) \mathrm{mmol} / \mathrm{l}$, respectively, $\mathrm{p}<0.05$ ) as compared with a single match at the end of exercise. Five minutes after repeated squash mean serum free fatty acid concentrations were higher $(1.63(0.34)$ and $0.84(0.16)$ meq/l, respectively, $p<0.05)$ as compared with a single match. The mean plasma magnesium concentration tended to fall after squash but there was no difference between repeated squash and a single match. Poorer anaerobic fitness was associated with greater increments in lactate.

We conclude that squash is associated with changes in potassium, lactate, and free fatty acid concentrations that may be arrhythmogenic, and repeated squash over a 48 hour period is associated with greater increments in free fatty acid and lactate concentrations.
Hypertensive left ventricular hypertrophy and strain: evidence for a myocardial structural abnormality

S D Pringle, P W Macfarlane, J H McKillop, A R Lorimer, F G Dunn

University Departments of Medicine and Medical

Cardiology, Royal Infirmary, Glasgow, and

Department of Medical Cardiology, Stobhill

General Hospital, Glasgow

Factors other than blood pressure contribute to the increased mortality in hypertensive patients with electrocardiographic left ventricular hypertrophy (LVH) and strain. A study was therefore undertaken to assess the degree of myocardial fibrosis as determined by echo amplitude analysis. A mathematical index of colour encoded pixel intensity was calculated in 24 normal subjects, 37 athletes with LVH, and 65 hypertensive patients with varying degrees of ECG abnormality (12 normal ECG, 16 ECG-LVH (voltage only), and 37 ECG-LVH and strain, of whom 19 had coronary arteriography (seven with coronary artery disease (CAD) and 12 with normal coronary arteries). The mean (SD) posterior wall echo amplitudes were similar in all groups (normals $20.3(2 \cdot 2)$, athletes $20.2(3.7)$, hypertensives 21.09 (3.63). There were, however, significant differences in the septum, with LVH and strain patients having higher amplitudes $(27.02(8.6)$ than normal subjects $(21.5(3.6))$, athletes $(22 \cdot 7(3.42))$, and hypertensive patients with a normal ECG (22.4 (2.9)) (all $\mathrm{p}<0.05)$. These increases did not correlate with left ventricular mass $(r=0.32, N S)$ or the presence of CAD.

Echo amplitude analysis of the septum separates not only physiological from pathological hypertrophy but also hypertensive patients with $\mathrm{LVH}$ and strain from those with a normal ECG. As this abnormality is not specifically related to increased mass or $\mathrm{CAD}$ it may be an additional contributory factor to the known increased mortality in hypertensive patients with LVH and strain.

Nocturnal decrease in fibrinolysis is consistent with peak onset of myocardial infarction, sudden cardiac death, and stroke

Felicita Andreotti, M I Khan, A deBart, D Hackett, G J Davies, A Maseri, C Kluft

Royal Postgraduate Medical School, Hammersmith Hospital, London, and Gaubius Institute TNO, Leiden, The Netherlands 
The frequency of onset of myocardial infarction, sudden cardiac death, and stroke exhibits a circadian variation with a peak during the morning hours. We determined the circadian variation of plasma fibrinolytic factors from three hourly venous samples taken from six healthy non-smoking volunteers aged 23 to 39 years over a typical 24 hour period of normal activity. The activities of tissue type plasminogen activator (tPA) and the fast acting plasminogen activator inhibitor (PAI) were assessed by amidolytic spectrophotometric assays. A pronounced and opposite circadian variation in TPA and PAI activities was observed. The curves were sinusoidal with a peak tPA activity of $787(391) \mathrm{mIU} / \mathrm{ml}$ (mean (SD)) and lowest PAI activity of $13(9.7) \%$ pooled plasma inhibition occurring at 1800 hours. The lowest tPA activity of $3.85(5.6) \mathrm{mIU} / \mathrm{ml}$ coincided with a peak PAI activity of $178(62) \%$ at $0300 \mathrm{~h}$. The timing of peaks and nadirs was consistent among individuals ( $S D=1 \mathrm{~h}$, best fit curve) and the differences between mean peak and nadir activities were significant for both tPA $(p<0.005)$ and for PAI $(p<0.002)$. Peak to nadir change, however, in tPA and PAI varied considerably from one subject to another $(47.3$ to $242 \mathrm{mIU} / \mathrm{ml}$ for tPA, 115 to $274 \%$ for PAI).

The occurrence of greatest fibrinolytic inhibition with lowest fibrinolytic activity in the early morning hours suggests that reduced endogenous fibrinolysis may be a possible mechanism contributing to the onset of thrombosis in acute myocardial infarction, sudden cardiac death, and stroke.

\section{Risk factors in prediction of heart attacks in} men with pre-existing ischaemic heart disease

A N Phillips, A G Shaper, S J Pocock, Mary Walker, $P$ W Macfarlane

Royal Free Hospital School of Medicine, London, and Glasgow University

There are strong associations between serum total cholesterol, blood pressure, and cigarette smoking, and the incidence of heart attack. In survivors of myocardial infarction (MI) the role of these risk factors is apparently diminished. In the British Regional Heart Study in 6.2 years average follow up, 336 of the $\mathbf{7 7 3 5}$ men have suffered a heart attack, fatal or non-fatal. Of the middle aged men $1968(25 \%)$ had evidence of pre-existing ischaemic heart disease (IHD). The men were grouped into (a) no evidence of pre-existing IHD, (b) those with evidence of preexisting IHD other than definite MI, and (c) definite previous MI. After standardisation for age, attack rates for IHD were determined for each of the three groups for varying levels of the risk factors. Cigarette smoking is strongly associated with attack rate in group (a). In groups (b) and (c) the relation persists but is reduced to borderline significance. For systolic blood pressure, the strong relationship with risk in group (a) is almost as strong in group (b), but disappears in group (c). For serum total cholesterol, the strong association with attack rate in group (a) diminishes through group (b) to group (c) but remains appreciable and highly significant.

This observation, together with the results from previous studies and trials, suggests that reduction of total serum cholesterol in men with evidence of IHD may be at least as important as the control of blood pressure and the cessation of smoking.

Serotonin antagonism prevents myocardial infarction and death from coronary artery disease

M I M Noble, A J Drake-Holland

King Edward VII Hospital, Midhurst, and St George's Hospital, London

Serotonin may be an important mediator in the process of coronary thrombosis. It is plentiful in platelets and is released in large quantities during coronary thrombosis. It mediates the initial platelet thrombosis and vessel spasm and amplifies the effects of other mediators. A serotonin antagonist should therefore be a potential inhibitor of the whole process of coronary occlusion. Thirty eight patients with critical arterial stenoses shown by coronary angiography were divided into a control (aspirin treated) group and a ketanserin (serotonin antagonist) treated group in an open trial. The treatment that the patients would normally have undergone anyway,-for example, angioplasty, or coronary artery bypass grafting-was allowed to proceed in the usual way with both groups. Allocation according to age and severity of disease was carried out by a non-clinician who did not know the patients. Controls: $n=19$, age $67 \cdot 1(8 \cdot 4), 13$ men and six women, affected vessels: one $(n=2)$, two $(n=7)$, three $(n=9)$; unstable angina, $n=2$, stable angina, $\mathrm{n}=17$; angioplasty, $\mathrm{n}=3, \mathrm{CABG}, \mathrm{n}=7$. Treated: $\mathrm{n}=19$, age $64.4(7 \cdot 4), 17$ men and two women, involved vessels: one $(n=2)$, two $(n=5)$, three $(n=11)$; unstable angina, $n=2$, stable angina, $n=17$; angioplasty, $n=2, C A B G, n=8$. During the two year follow up period none of the patients treated with ketanserin have had any major ischaemic episodes; they are symptom free or in the 
case of those with stable angina they remain stable. There have been two deaths in the control group, two myocardial infarctions, two coronary artery bypass graft thromboses, and one thrombotic arterial occlusion at the site of angioplasty requiring CABG.

These results constitute a significantly better outcome in the treated group $\left(\mathrm{p}<0.05, \chi^{2}\right.$ test). It seems unlikely that this can be accounted for on the basis of different sex distribution between the groups.

\section{Pre-existing coronary stenoses in patients with first myocardial infarction are not necessarily severe}

D Hackett, G Davies, S Chierchia, A Maseri Royal Postgraduate Medical School, Hammersmith Hospital, London

The sudden, often unheralded, onset of symptoms in acute myocardial infarction suggests that preexisting coronary stenoses susceptible to acute thrombosis in the infarct related artery may not necessarily be severe. We studied 60 consecutive patients aged 27-78 years within six hours of the onset of their first acute myocardial infarction by performing coronary arteriography before and after intracoronary thrombolytic treatment. We assessed previous symptoms and collateral vessel filling of the occluded infarct vessel before thrombolysis and measured the severity of residual coronary stenoses after successful thrombolytic recanalisation using computerised angiography. Recanalisation was achieved in 48 patients with a residual stenosis diameter obstruction of $58 \cdot 1(10 \cdot 8) \%$ (mean (SD)) and a minimum absolute lumen of $1.10(0.3) \mathrm{mm}$ diameter. A residual stenosis of $<50 \%$ was present in $12(25 \%)$ patients but none had collateral filling, between $50-75 \%$ in $34(71 \%)$ patients of whom 12 had collateral filling $(p=0.04)$, and between $75-100 \%$ in two $(4 \%)$ of whom one had collateral filling. Collateral filling was present in six of the 12 $(20 \%)$ patients who did not recanalise. The symptoms before presentation did not correlate with the severity of the residual infarct related stenoses after thrombolysis, and the presence of collateral filling was not related to the preceding symptoms.

Residual coronary stenoses after thrombolysis in patients with their first acute myocardial infarction are not necessarily severely obstructive. Mild residual stenoses are associated with no short term collateral filling of the occluded artery.

\section{Endothelial integrity in human atherosclerotic coronary arteries}

\section{J Davies, J Pepper, N Woolf, P Rowles \\ St George's and the Middlesex Hospital Medical Schools, London}

In experimental atheroma induced by lipid feeding the interplay of endothelial damage with platelets and macrophages is important in early plaque growth. Because endothelial integrity cannot be studied in specimens from necropsy or endarterectomy it is uncertain whether similar endothelial damage occurs over human plaques. In transplantation the coronary arteries can be perfused instantly with glutaraldehyde giving ideal endothelial preservation, free from artefact, for scanning electron microscopy. The right coronary arteries of six patients undergoing transplantation were studied. In two the artery was angiographically normal, in one irregular in outline, and three had appreciable stenosis. None of the patients had raised concentrations of cholesterol and triglycerides. In four of the six arteries the endothelial surface showed widespread focal abnormalities ranging from adhesion and migration of monocytes to loss of individual endothelial cells. Larger areas of endothelial denudation with exposure of underlying collagen were also seen. Loss of endothelial cells was associated with accumulation of macrophages, on and deep to the surface, as well as adhesion of platelets to the exposed subendothelial tissue. Endothelial damage was not related specifically to stenotic segments.

The results suggest that some patients with coronary atheroma have widespread focal endothelial cell abnormalities and that human atheroma in this regard, even in normolipidaemic patients, is similar to animal models. The morphological abnormalities of endothelial cells may indicate a more widespread functional change including loss of endothelial derived relaxant factor and altered vasomotor responses.

\section{Is the in-hospital course of women after a first myocardial infarction different from that in men?}

\section{K Robinson, $\mathbf{R}$ Conroy, $\mathbf{R}$ Mulcahy \\ St Vincent's Hospital, Dublin, Ireland}

The course of myocardial infarction in women has not yet been fully elucidated and it has been suggested that their prognosis is worse than that of men. 
We studied risk factors and the course in hospital in 337 women and 658 men admitted with a first myocardial infarction or episode of acute coronary insufficiency. Women were older than men $(\mathrm{p}<0.01)$ and smoked less $(\mathrm{p}<0.0001)$, but more were hypertensive $(p<0.05)$ and mean serum cholesterol concentration was higher $(p<0.05)$. Logistic regression analysis showed that these risk factor differences, with the exception of hypertension, were not due to the age difference between the sexes. Women were more likely to be admitted with acute coronary insufficiency $(\mathrm{p}<0.05)$, and this difference was attributable to the differing risk factor distribution between the sexes. Women with infarctions had a higher rate of complications $(p<0.0001)$. Their death rate $(12.6 \%)$ was significantly higher than that among men in whom it was $6.5 \%(p=0.02)$. When the confounding effect of age was removed using logistic regression, sex no longer correlated with complications in hospital (NS) or mortality (NS).

Although risk factor differences exist between the sexes, gender per se does not influence the course in hospital of a first episode of acute coronary heart disease. Previously reported differences may be due to the effect of confounding variables, notably age.

\section{Intracoronary epoprostenol: effects on coronary artery calibre}

\section{J Wilson, N P Silverton, $M$ W Baig, E J Perrins, D R Smith, J A Davies, C R M Prentice Departments of Cardiology and Medicine, General Infirmary, Leeds}

Epoprostenol (prostacyclin, $\mathrm{PGI}_{2}$ ) is a prostaglandin with vasodilatory and platelet inhibitory properties and could, therefore, be of benefit during coronary artery angioplasty. The aim of this study was to assess both the safety and angiographic vasodilatory capacity of intracoronary (I/C) epoprostenol. Sixteen patients were studied. After coronary angiography, the view displaying the left epicardial coronary arteries (ECA) most clearly was selected as a control. I/C epoprostenol was then given and the angiogram repeated in the chosen view. This was repeated with a higher dose of epoprostenol and again following $\mathrm{I} / \mathrm{C}$ isosorbide dinitrate (ISDN). All angiograms were coded and analysed blind. ECA calibre was measured from traced projections of the angiograms. Blood pressure, heart rate, and ECG were recorded throughout. The first two patients received epoprostenol infusions of 2.5 and $5.0 \mathrm{ng} / \mathrm{kg} / \mathrm{min}$ to assess safety, and no untoward reactions occurred. The next 10 patients had epoprostenol infusions of 5.0 and $7.5 \mathrm{ng} / \mathrm{kg} / \mathrm{min}$ followed by I/C ISDN. No haemodynamic disturbances occurred and ECA calibre did not change with epoprostenol [mean ECA diameter $\mathrm{mm}$ (SD): control $2.85(0.62), 5.0 \mathrm{ng} / \mathrm{kg} 2.80(0.61), 7.5 \mathrm{ng} / \mathrm{kg}$ $2 \cdot 80(0 \cdot 54))$, but did increase with ISDN $(3 \cdot 17(0 \cdot 36))$ $(\mathrm{p}<0.02)$. The last four patients had epoprostenol infusions of 7.5 and $10 \mathrm{ng} / \mathrm{kg}$ followed by I/C ISDN, and two patients became hypotensive. ECA calibre did not change significantly [control $3.5(0.45)$, $7.5 \mathrm{ng} / \mathrm{kg} / 2.96(0.81), 10 \mathrm{ng} / \mathrm{kg} 3.45(0.96)$, ISDN $3.20(0.61)$ ]. Eight patients developed tall $T$ waves on the ECG during epoprostenol infusions but no arrhythmias occurred.

The results suggest that clinically tolerable doses of I/C epoprostenol are not potent dilators of the ECAs, and that this route of administration is unlikely to be of use during coronary angioplasty.

\section{Does hyperventilation have any cardiac effect in patients with chest pain?}

Leisa Freeman, P G F Nixon

Cardiac Department, Charing Cross Hospital, London

Intermittent acute episodes of hyperventilation have been documented in patients with angina like chest pain and both atheromatous and normal coronary arteries. A direct cardiac effect has been suggested. Fourteen consecutive patients undergoing cardiac catheterisation were studied. Seven had coronary artery disease (CAD) and seven normal coronary arteries (NCA). Medication was withdrawn before the study. Atenolol $100 \mathrm{mg}$ was given four hours before the study and temporary pacing started at $70 \mathrm{bpm}$ in order to avoid the sympathomimetic effects of hyperventilation. All patients hyperventilated for three minutes and a respiratory alkalosis resulted: mean arterial pH 7.53; mean $\mathrm{PaCO}_{2}$ of $26(4 \cdot 6) \mathrm{mm} \mathrm{Hg}$. Coronary sinus $\mathrm{PCO}_{2}$ fell by a mean of $17(4.8) \mathrm{mmHg}$. Arterial - venous oxygen $\left(\mathrm{A}-\mathrm{Vo}_{2}\right)$ content widened from $9 \cdot 1(1.2)$ to 10.1 (1.0) $(\mathrm{p}<0.03)$ in patients with $\mathrm{CAD}$ and myocardial oxygen extraction increased by $4.5 \%$. In NCA patients $\mathrm{A}-\mathrm{Vo}_{2}$ content difference narrowed from $10.0(2 \cdot 1)$ to $8.6(1.0)(\mathrm{NS})$ and oxygen extraction fell by $6.4 \%$. Arterial lactate concentration increased in response to $\mathrm{HV}$ in all subjects, but $\mathrm{CS}$ lactate increased only in the CAD patients $(0.63(0.07)$ to 0.8 $(0.7), \mathrm{p}<0.02) . \mathrm{dP} / \mathrm{dt} \max$ was measured using a fibreoptic Camino catheter and increased significantly in all patients following $\mathrm{HV}$; left ven- 
tricular end-diastolic pressure only rose significantly $(13.7(2.4)$ to $17.4(2.0) ; p<0.03)$ in the patients with CAD.

Thus coronary flow limitation may occur in response to hyperventilation in patients with CAD, and ventricular function may deteriorate.

\section{Free radical production and neutrophil} elastase in myocardial injury

D Bell, M Jackson, R M Dawkes, J Walker, J Dawes, A L Muir

Department of Medicine, Medical Research Council and Scottish National Blood Transfusion Service Blood Components Assay Group, Royal Infirmary, Edinburgh

Animal models of myocardial infarction suggest that the neutrophil, by releasing oxygen derived free radicals and elastase, may potentiate myocardial damage. We measured plasma lipid peroxidation products (octadecadienoic acid) as a measure of free radical production, and plasma neutrophil elastase concentrations as a measure of neutrophil activation, in normal controls, patients with stable angina, and sequentially in patients with acute myocardial infarction, over $48 \mathrm{hrs}$. Thirty five controls (mean age 35 , range $22-63$ years), 20 patients with stable angina (mean age 60 , range $42-72$ ) and 15 patients with acute infarction (mean age 58 years, range 35-73, peak creatine kinase (CK) concentration $1791(223) \mathrm{u} / 1)$. There was no difference in plasma octadecadienoic acid between controls (20.4 (1.15) $\mu \mathrm{mol} / \mathrm{l})$ and patients with angina $(23.5(2 \cdot 19) \mu \mathrm{mol} / \mathrm{l})$. Peak plasma octadecadienoic acid concentrations were significantly higher in patients with acute myocardial infarction (36.9 (3.33) $\mu \mathrm{mol} / \mathrm{l})$ compared with controls $(p<0.001)$ or those with angina $(p<0.01)$. There was no difference in plasma neutrophil elastase concentrations between controls $(21.1$ (1.91) $\mathrm{ng} / \mathrm{ml})$ and patients with angina $(26.6(2.04) \mathrm{ng} / \mathrm{ml})$. Peak plasma elastase concentrations were significantly higher in patients with myocardial infarction (58.3 (8.67) $\mathrm{ng} / \mathrm{ml})$ compared with controls $(p<0.001)$ and patients with angina $(p<0.001)$. Peak concentrations of octadecadienoic acid occurred earlier after the onset of chest pain (13.2 (2.0) hours) compared with peak concentrations of elastase (23.3 (3.6) hours) $(p<0.02)$.

While oxygen derived free radicals could derive from myocyte destruction, the elastase indicates degranulation of the neutrophil and confirms early activation of the neutrophil in myocardial infarction.
A comparison of exercise training and $\beta$ blockade on the chronotropic response to treadmill exercise in angina pectoris

\section{C Todd, D Ballantyne \\ Victoria Infirmary, Glasgow}

Twenty four patients with chronic stable angina were recruited and randomised into two groups. Both groups underwent three standard symptom limited exercise tests on a treadmill, the first for familiarisation purposes, the second to obtain baseline values, and the third after treatment with atenolol $100 \mathrm{mg}$ daily for seven days. $\beta$ blockers were stopped and group A started exercise training for 11 minutes daily at home with weekly hospital supervision (Canadian Airforce PBX programme). Both groups initially received nifedipine $10 \mathrm{mg}$ three times a day and were followed up for a year at which time all tests were repeated. Atenolol produced a $44 \%$ improvement in mean treadmill time for group A which was significant $(p=0.01)$. This was accompanied by an improvement in workload from 6 to 8 metabolic equivalents. Exercise training produced a much greater increase in treadmill time of $91 \%$ $(p=0.0007)$, a workload of 10 metabolic equivalents. This was significantly better than atenolol $(p=0.04)$. The heart rate at rest and at each stage of the exercise test was reduced following training but the degree of reduction was less than that when taking atenolol. Atenolol resulted in a significant fall in mean maximum double product, however, $(p=0.0002)$ while training produced a significant increase in mean maximum double product $(p=0.04)$. A non-significant deterioration in all the above measurements was seen in the control group. Drug usage among the training group decreased markedly while in the control group two patients required coronary artery bypass grafting.

Exercise training therefore produced changes in exercise tolerance superior to that achieved by $\beta$ blockade.

\section{Determinants of cerebral blood flow during cardiopulmonary bypass}

G Venn, K Sherry, T Treasure, S Newman, $M$ Harrison, L Klinger, P Ell

Middlesex Hospital and Medical School, London

In a study of the cerebral consequences of cardiopulmonary bypass (CPB) we have assessed the cerebral blood flow (CBF) by intra-arterial Xenon-133 clearance, using a Novocerebrograph 10a, before, 
during, and immediately after CPB. All 51 patients under the care of a single surgical team had elective coronary revascularisation by a standardised anaesthetic and surgical protocol. CBF fell significantly from before (median 22) to on bypass (median 20) (Wilcoxon, $\mathrm{p}<0.005$ ) and was significantly higher immediately after the bypass period (median 28) than before bypass (Wilcoxon, $p<0.001$ ). Scattergrams showed that CBF was independent of arterial pressure (BP) but was related to arterial carbon dioxide. The correlations between arterial carbon dioxide pressure and CBF before bypass $(r=0.46$, $\mathrm{p}<0.001)$ and after bypass $(r=0.46, \mathrm{p}<0.002)$ are very similar; while on bypass the correlation, although significant, is reduced. The median values for arterial carbon dioxide pressure were low throughout the study (before bypass, median 33; on bypass, median 28 ; and after bypass, median 36 ).

The maintenance of arterial carbon dioxide pressure within the normal range of $35-45 \mathrm{~mm} \mathrm{Hg}$ may minimise the risk of low CBF caused by hypocarbia which could theoretically be harmful in patients known to be at risk of both short and long term cerebral dysfunction.

\section{Long term evaluation of isolated Björk-Shiley mitral valve replacement}

I Th Fessatidis, K E Vassiliadis, P J Drury, D F Shore, J L Monro, J K Ross

Wessex Cardiothoracic Centre, General Hospital, Southampton

Between 1973 and 1985, 331 patients out of 356 survived isolated mitral valve replacement with Björk-Shiley valves (models MBRP-standard, MBRC-convex-concave and MMSMmonostrut). We followed up $292(88 \%)$ patients for up to 13 years, a total of 6620 patient-years, to determine the long term performance characteristics of these prostheses. At operation the mean age of the patients was 54 years (range 18-80), 70\% were women, and $59 \%$ were in atrial fibrillation. The mitral valve disease was rheumatic in $132(40 \%)$ and associated with endocarditis in $45(14 \%)$ patients. The early mortality was $5 \%$ and there were 54 late deaths $(19 \% ; 0.8 \%$ per patient-year) of whom 35 $(12 \%)$ were cardiac in origin with seven cases $(2 \%)$ of thromboembolism of the valve. Total 13 year valve related morbidity in 48 patients $(16 \%), 0 \cdot 7 \%$ per patient-year) was due to thromboembolism in $29(10 \%)$, prosthetic endocarditis in eight $(3 \%)$, anticoagulant related haemorrhage in five $(2 \%)$, and paraprosthetic regurgitation in six $(2 \%)$.
Reoperation was performed in five $(2 \%)$ because of prosthetic endocarditis (three patients, $1 \%$ ) or paraprosthetic regurgitation (two patients, $0 \cdot 7 \%$ ), all necessary within the first year after operation. No case of haemolysis or mechanical valve failure was recorded. Total 13 year actuarial estimates of survival were $58 \%$ and freedom from valve related morbidity $71 \%$.

The Björk-Shiley models MBRP, MBRC, and MMSM mitral valve prostheses show satisfactory durability without any mechanical failure over a 13 year period. The long term outcome of isolated mitral valve replacement with these models is associated with a low incidence of valve related complications.

\section{Conservative operations for congenital abnormalities of the mitral valve}

N K Bhatnagar, H S Joffe, S C Jordan, J D Wisheart Bristol Royal Infirmary, and Bristol Royal Hospital for Sick Children, Bristol

In congenital abnormalities of the mitral valve (MV), repair is the goal of surgical management. Between 1975 and 198710 patients aged between four months and 14 years (mean 56 months) underwent 11 operations, and follow up was both clinical and by cross sectional and Doppler echocardiography. Two children had isolated mitral incompetence, each with a giant left atrium causing airway obstruction and pulmonary complications; both valves were repaired (pericardial patch advancement of posterior cusp, repair of cleft anterior leaflet, annuloplasty, and enhancement of the commissural coaption). After seven and five years, respectively, both are well, free of murmurs, and have normal heart size and lung fields. Seven children had mitral stenosis (MS); six had supravalvar rings (SVR) and one had a parachute valve. Five had "forme fruste" of the Shone complex, each showing either two or three of its four features. Conservative operations were successful in six cases and mitral valve replacement (MVR), was required in one patient with SVR leading to the only operative death in this series. Associated procedures for subaortic stenosis were carried out in three patients. Of the six survivors one is abroad and lost to follow up and one required MVR five years later. After two to 138 months (mean 49 months) of follow up four are in grade I and one in grade II New York Heart Association classification; three have soft systolic murmurs, one has cardiomegaly, and valve thickening on Doppler. The final patient had redundant mitral tissue on the anterior leaflet which filled 
in systole and caused subaortic obstruction. This tissue was excised and the valve repaired, and the patient remains well and asymptomatic.

Conservative surgery was possible and had a good outcome in nine out of 10 cases with severe congenital MV anomalies.

Initial experience with seeding of a neointima in a rough surface left ventricular assist device

T R Graham, J A C Chalmers, I W Weir, $S$ Withington, $\mathrm{P}$ Colvin, Denise

Syndercombe-Court, C L Berry, C T Lewis

Department of Cardiothoracic Surgery, Institute of Pathology, The London Hospital, London

Development of an implantable left ventricular assist device (LVAD) for prolonged circulatory support has been impeded by the lack of a suitable biomaterial to interface with circulating blood. Progressive thrombus formation has been noted on the surface of LVADs lined with smooth nonthrombogenic polymers designed to attract a fibrin coagulum. The Thermedics pneumatic LVAD has a rough surface lining made of an artificial collagen like matrix Biomer. Calf foetal fibroblasts in cell culture have previously been shown to proliferate and produce collagen freely. Three LVADs were seeded with a mean of 59 million of these cells before insertion into three month old calves, mean weight $76 \mathrm{~kg}$. These devices were inserted for six hours, nine days, and three months. Histological and electron microscopic examination confirmed the presence of a smooth neointima containing two layers of viable active fibroblasts immersed in collagen and coagulum on the dynamic pusher plate surface of the LVAD. The seeded fibroblasts seem to have rapidly encouraged the laying down and adherence of this smooth neointima. Initially the static metal housing was covered with a thin layer of coagulum with no visible active fibroblasts. Consequently, our seeding technique included an initial prime with autologous plasma to improve fibroblast adhesion. Microscopic examination has confirmed this. There has been no thrombus formation observed within the retrieved LVADs, neither has there been any evidence of systemic micro or macro embolisation at necropsy.

This lining offers the potential for prolonging the thromboembolic free function of LVADs.
Association between coronary artery collateral circulation and post infarction ventricular septal defect

\author{
J D Skehan, Catherine Carey, $M$ de Belder, I Weir, \\ T Graham, P G Mills \\ The London Hospital, London
}

Ventricular septal defect (VSD) after myocardial infarction (MI) was seen in 28 patients over four years. The mean age ( 67 years) and male/female ratio $(1 \cdot 3: 1)$ differed from that usually associated with MI. Most patients (24 of 28 ) presented within 14 days of MI after a stable interval. All had heart failure and most (24) were hypotensive. Inotropic drugs were used in 21 and aortic balloon counterpulsation in 16. The angiographic features for these 28 VSD patients were compared with 123 stable patients also studied early after MI. The VSD group had strikingly fewer major collaterals to the infarct related artery $(4 \%$ and $63 \%$ respectively, $p<0.01)$ although minor collaterals occurred equally $(18 \%$ and $15 \%)$. Total occlusion of the infarct vessel was seen in 23 $(82 \%)$ and aneurysm formation in $17(67 \%)$. Additional coronary disease (stenosis $>70 \%$ ) was seen in 17 but only 10 had aortocoronary grafts (eight to a single vessel). Of 27 patients undergoing surgery eight had multiple VSDs, 17 patch repairs, 15 aneurysm resection, and two required reoperation. Overall mortality was $25 \%$ with one preoperative death, four perioperative deaths (one after reoperation), and two sudden deaths, one at three and one at seven months. Of the survivors 16 are well, four have symptomatic heart failure, one has dementia, and none has angina.

We conclude that a lack of collaterals to the infarct vessel suggests that a different balance of coronary circulation may pre-exist in older patients who have developed a VSD from other survivors of MI. Early operation with a low rate of grafting of non-infarct vessels gave a relatively low mortality.

\section{Internal mammary artery grafting increases the incidence of shoulder girdle pain after cardiac surgery
$R$ Watts, $R$ Davies, $T$ Treasure
Department of Medicine, Whittington Hospital, London, and Department of Cardiothoracic
Surgery, Middlesex Hospital, London

Exposure of the heart through median sternotomy requires distortion of the chest wall and shoulder 
girdle. The increased popularity of the internal mammary graft (IMA) and in particular the use of the Favorolo retractor may have added to the frequency of shoulder pain. We prospectively studied 53 patients undergoing cardiac surgery ( 17 coronary surgery with IMA, 14 without, 15 valve replacements, and seven other combinations) all free of shoulder pain before operation. All patients were examined six weeks after operation and most were followed up with clinical examination (21) or questionnaire (29) at six months. Pain of cardiac and pulmonary origin was excluded on clinical grounds. Shoulder symptoms were reported by 29 patients, 13 of 17 in the IMA group and 16 of 36 of the others $(p=0.02$, Fisher's exact test). The operative detail was not known at the time of evaluation. A specific diagnosis referrable to the shoulder girdle was made in six patients (two had shoulder capsulitis, two had supraspinatus tendonitis, and one had acromioclavicular and one sternoclavicular strain) and was of non-specific origin in the remaining 23. Pain started within three months of operation in 23 patients, lasted four to six months in 11 patients and interfered with activities of daily living in 13 (seven IMA, six others (NS, Fisher's exact test)). Four patients required physiotherapy or intra-articular injections.

Accurate diagnosis of the source of pain may lead to specific treatment. Awareness of the problem should reduce unnecessary or excessive retraction.

\section{Initial trials of a transtelephonic defibrillator}

G W N Dalzell, S R Cunningham, S Pruzina, $H$ Magee, J Anderson, A A J Adgey

Regional Medical Cardiology Centre, Royal Victoria Hospital, Belfast

For patients with out of hospital ventricular fibrillation, the time until attempted defibrillation is one of the major determinants of survival. Earlier defibrillation can be facilitated by remote telephonic control of a defibrillator placed in the patient's home. We have assessed the efficacy of transtelephonic defibrillation (TTD) in 26 cardioversions (CV) (25 elective, one emergency) of atrial fibrillation $(n=22)$, and ventricular tachycardia $(n=4)$ performed in 23 patients ( 16 men, seven women) aged 46-81 (mean 61 years). The patient unit (PU) contains a microprocessor, microphone, defibrillator, and ECG/defibrillator pads applied to the patient's chest. The PU is connected to a standard telephone and once activated automatically dials and activates the remotely sited base station (BS) establishing ECG transmission and two way voice commu- nication. The physician at the BS diagnoses the $\overrightarrow{\vec{F}}$ rhythm and controls defibrillator synchronisation, charging, and discharge, having confirmed anaesthesia. Accuracy of the ECG/defibrillator pad position is assessed by monitoring of transthoracic impedance at the BS. The time for activation of the BS by the PU ranged from 18 to 24 seconds (mean 20.5 ), and in all $\mathrm{CV}$ clear voice communication and ECG signals were established. A total of $75 \mathrm{DC}$ shocks $(50-360 \mathrm{~J})$ were safely administered with no adverse effects and were successful in 24 of $26 \mathrm{CV}$. The average discharge delay time from BS to PU was $2 \cdot 6$ seconds (range $1 \cdot 5-5$ ).

TTD has the potential for more rapid defibrillation of patients with ventricular fibrillation and minimises patients' or spouses' interaction with the system.

\section{Surgery for "inoperable" ventricular tachycardia}

J P Bourke, Suda Tansuphaswadikul, J C Cowan, C J Hilton, Janet McComb, R W F Campbell Cardiothoracic Unit, Freeman Hospital, Newcastle upon Tyne

Intraoperative arrhythmia activation mapping underlies the impressive success of surgery for sustained uniform ventricular tachycardia. Unstable arrhythmias and those intraoperatively noninducible, however, are not amenable to activation mapping and have been considered "inoperable". We propose that fragmentation mapping in sinus rhythm can direct surgery in such arrhythmias. In 18 of 51 patients operated at this unit, intraoperative arrhythmia mapping was impossible because of noninducibility $(n=15)$ or unstable configuration $(n=3)$. Surgery comprised endocardial resection of all areas showing fragmented local electrograms in sinus rhythm ( $>100 \mathrm{~ms}$ duration). Mean patient characteristics included: age 52 years; arrhythmic episodes 18 (range 2-200); antiarrhythmic drug failures 4; left ventricular ejection fraction $33 \%$. There were five $(28 \%)$ early postoperative deaths (heart failure, three; sudden, one; metabolic, one) and one early recurrence of arrhythmia. There have been two late non-arrhythmic deaths and no further arrhythmia recurrences with mean follow up of $22(19)$ months. No patient requires long term antiarrhythmic drug treatment.

Fragmentation mapping successfully extends the surgical option to arrhythmias previously considered "inoperable" with results that compare favourably with those for arrhythmias in which operation was directed by activation mapping. 
Surgical correction of atrioventricular junction reentrant tachycardia

\author{
P L Weissberg, R W Harper, A Broughton, \\ G Shardey, A Pitt
}

Alfred Hospital, Melbourne, Australia

It has been assumed that the reentry circuit in atrioventricular junction reentrant tachycardia (AVJRT) is entirely intranodal. It has recently been proposed, however, that extranodal atrial fibres may form part of the reentry circuit and that surgical division of these fibres will abolish AVJRT while preserving A-V conduction. We have performed this operation on eight patients, three men, mean age 21.8 (range 13-34) years, with recurrent AVJRT. At preoperative electrophysiology study AVJRT was classified as type A: earliest atrial activation recorded by the His catheter (seven patients) and type B: earliest activation recorded in the proximal coronary sinus (one patient). Four patients also had A-V accessory pathways (AP). All patients underwent $A-V$ nodal dissection-anterior for type $A$ and posterior for type B including map-guided division of AP also where appropriate. All APs were successfully divided. V-A conduction was abolished in five patients. One patient had long term $\mathrm{A}-\mathrm{V}$ dissociation requiring a permanent pacemaker. $\mathrm{A}-\mathrm{V}$ conduction was preserved in the remainder but was apparently modified in most. Only one patient (BS) had a recurrence of AVJRT. In this patient AVJRT continued despite complete atrial dissociation during tachycardia implying an entirely intranodal circuit. AVJRT could not be induced in any of the other patients at a postoperative electrophysiological study.

These results confirm that this operation can abolish AVJRT without destroying A-V conduction. The findings in BS suggest, however, that its success is caused by modification of intranodal conduction rather than division of a specific extranodal pathway.

\section{Concealed pre-excitation does not protect against rapid ventricular response to atrial fibrillation}

K Robinson, E Rowland, D M Krikler

Royal Postgraduate Medical School, Hammersmith Hospital, London

The Wolff-Parkinson-White syndrome has a typical electrocardiographic appearance. When preexcitation is intermittent, or disappears during exer- cise testing or other manoeuvres, the risk of a rapid ventricular response to atrial fibrillation is thought to be low. The discovery of unsuspected accessory pathways in several reports on sudden cardiac death suggests otherwise. Among 108 patients with preexcitation we have identified three in whom anterograde conduction across the accessory pathway was evident only with atrial pacing or with development of atrial fibrillation. One patient presented with near syncope and the others with palpitations. All had normal electrocardiograms in sinus rhythm. Although re-entrant atrioventricular tachycardia was the initial arrhythmia in one, the others had presented with atrial fibrillation with complexes suggesting pre-excitation. At electrophysiological study incremental right atrial pacing confirmed the presence in all of an accessory pathway which was located on the left side. The range of mean ventricular responses during induced atrial fibrillation was $200-280 /$ minute. One patient had enhanced atrioventricular nodal conduction, and one also had mitral valve disease with an enlarged left atrium.

Atrial fibrillation may be responsible for exposure of obvious pre-excitation on the surface electrocardiogram even when this is not visible in sinus rhythm. Important contributing factors to this may be the location of the pathway, the rapidity of atrioventricular nodal conduction, and atrial disease. The absence of resting pre-excitation does not exclude the possibility of dangerously high ventricular rates.

\section{d-Sotalol-induced infra-His block: evidence of class III activity}

Janet McComb, J P Bourke, J N Ruskin, H Garan, R W F Campbell

Freeman Hospital, Newcastle upon Tyne

d-Sotalol prolongs action potential duration, and has effects consistent with class III activity, prolonging atrial and ventricular refractoriness. Its effect on the His-Purkinje system is not known. To study this, measurements of AV nodal and His-Purkinje function were made before and after intravenous infusion of d-sotalol $1-2 \mathrm{mg} / \mathrm{kg}$ ) in 16 patients. The $\mathrm{AH}$ interval increased from $100(32)$ to $113(34) \mathrm{ms}(\mathrm{p}<0.05)$. The AV nodal effective and functional refractory periods prolonged from $342(65)$ to $391(85) \mathrm{ms}$ $(p<0.01)$ and from $429(74)$ to $465(87) \mathrm{ms}(\mathrm{p}<0.01)$. The functional refractory period of the His-Purkinje system was prolonged from $429(71)$ to $471(85) \mathrm{ms}$ $(\mathrm{p}<0.001)$. The $\mathrm{HV}$ interval at rest did not change (49(11) to 53(12) ms, NS) but HV prolongation was shown after either atrial extrasystoles or by rapid 
atrial pacing, after but not before d-sotalol infusion in six patients. Second degree infra-hisian block was shown in four patients, only after d-sotalol infusion.

d-sotalol therefore has pronounced effects on the His-Purkinje system, increasing refractoriness in all 16 patients and causing infra-hisian block in 4 of 16 . Similar effects have also been observed during amiodarone treatment, suggesting that prolongation of His-Purkinje refractoriness and infra-hisian block may be markers of class III activity.

Formation, transmission, and importance of high pressure shock waves during catheter ablation

A D Cunningham, E Rowland, A Ahsan, A F Rickards National Heart Hospital, London

High pressure waves after catheter ablation shocks have been suggested as both partially therapeutic and potentially damaging. We investigated shock wave formation by delivery of high energy discharges in a saline tank, with voltage, current, and photographic recording, and high pressure, high frequency manometry. Standard defibrillator impulses arced above $10 \mathrm{~J}$ (cathodal) and $15 \mathrm{~J}$ (anodal). Arcing was always associated with rapid gas production near the electrode and transient reduction in current flow. Peak pressure increased linearly with energy above this threshold. The slope was greater for anodal pulses, and thus very high energy $(>200 \mathrm{~J})$ anodal discharges produced the greater shock wave. Peak pressure at $5 \mathrm{~cm}$ was $5 \mathrm{~atm}$ for a $200 \mathrm{~J}$ cathodal or anodal shock, and decreased linearly with the inverse of distance from the electrode. Mean propagation velocity was $1594(14) \mathrm{m} / \mathrm{s}$. Perspex and tissue barriers were introduced between electrode and transducer. Perspex completely absorbed the shock wave, but tissue barriers up to $15 \mathrm{~mm}$ thick did not attenuate the shock. Thus shock wave energy is not absorbed by tissue, and direct barotrauma cannot occur. All shock waves had a secondary negative pressure wave, however, which induced gaseous microbubbles by cavitation. The collapse of these bubbles may contribute to diffuse myocardial damage, while rupture of myocardial structures is probably due to localised rapid gas production. Using a new energy source and catheter, four ablations ( $2 \mathrm{His}$ bundle, 2 VT) have been successfully carried out in man without arcing (individual shock energies $2-40 \mathrm{~J}$ ).

Direct barotrauma does not appear to exist, and secondary mechanical damage, while potentially rel- evant for unwanted side effects, is unnecessary for therapeutic ablation. A new completely non-arcing system may greatly increase safety margins in ablation.

Silent myocardial ischaemia after myocardial infarction: incidence and importance

J D Skehan, $M$ de Belder, C W Pumphrey, M T Rothman, P G Mills

The London Hospital, London

The incidence and importance of silent ischaemia after myocardial infarction (MI) is not clear. We defined silent ischaemia as asymptomatic ST segment depression on a submaximal exercise test (SMET). Patients studied were consecutive stable survivors of MI who had an early SMET and were followed up for one year. There were 67 patients with silent ischaemia (group I), 37 with angina and ST depression (group II), and 123 with a negative SMET (group III). The mortality in groups I $(9 \%)$ and II $(11 \%)$ were similar, and significantly higher than in group III $(0.9 \% \mathrm{p}<0.005)$. Groups I and II did not differ in the extent of coronary disease, the degree of collateral circulation to the infarct related vessel, the severity of left ventricular damage, or the rate of further MI. Fewer group I patients had $Q$ wave infarction and fewer complained of angina or underwent coronary artery surgery in the first year. The frequency of further MI or angina was similar in groups I and III. More group I patients had inferior $\mathrm{MI}$ and more had coronary artery surgery. Half the excess mortality in group I over III was due to sudden death (three of six).

We conclude that silent ischaemia occurs often in stable patients after MI $(>25 \%)$ and carries an increased mortality over patients without ischaemic changes on SMET.

Frequency and characteristics of silent ischaemia in patients with coronary artery disease

D Mulcahy, J Keegan, A D Cunningham, P A Crean, A A Quyyumi, C Wright, K M Fox National Heart Hospital, London

We studied 112 unselected patients ( 88 men), mean age 55 (range 35-75) with documented coronary artery disease ( 37 one vessel disease, 24 two vessel disease, 51 three vessel disease) using exercise elec- 
trocardiography and ambulatory ST segment Holter monitoring. Patients underwent 24-48 hours (total 4721) of ambulatory monitoring off all anti-anginal treatment. Seventy one patients had a total of 454 episodes of ST segment change (342 painless, 112 painful) during monitoring. Eleven had only painful episodes, 25 only painless, and 34 had both. Forty two patients had no ST changes. Episodes of silent ST segment change occurred throughout the day, but predominantly between 7 am and $8 \mathrm{pm}$, with a peak between 12 noon and $1 \mathrm{pm}$. There was no association between total or silent episodes of ST segment change and severity of coronary artery disease. Silent episodes were most frequent in patients with exercise tests positive at a low work load ( $<6$ minutes); those who developed ST segment depression at high work loads ( $>9$ minutes) rarely had silent ST segment changes on ambulatory monitoring. The mean number of all episodes of ST segment change per patient per day was $2.31(1.74$ silent); only 11 patients, however, $(10 \%)$ had $49 \%$ of all episodes recorded, and $32(29 \%)$ had $82 \%$. For silent ischaemia, 11 patients $(10 \%)$ had $54 \%$ of episodes, and 39 patients $(35 \%)$ had $93 \%$.

Of all episodes of ST segment change on Holter monitoring in patients with coronary artery disease $75 \%$ are silent and occur particularly in those with a definitely positive exercise test. Fifty three patients (47\%) had no evidence of silent ST change, and only $25(22 \%)$ had silent ST changes alone on Holter monitoring. It cannot therefore be concluded that most patients with ischaemic heart disease have frequent silent ischaemia, as has been previously assumed.

\section{Prognostic implications of silent ischaemia} on exercise testing after myocardial infarction

\author{
J P Fox, D P Murray, M Salih, M K Davies, \\ W A Littler, R G Murray \\ Department of Cardiovascular Medicine, \\ East Birmingham Hospital
}

The frequency and prognostic implications of silent ischaemia were investigated in 359 survivors of myocardial infarction. Predischarge exercise testing was performed following a modified Bruce protocol. Silent ischaemia was defined as the presence of $\geqslant 0 \cdot 1$ $\mathrm{mV}$ horizontal or downsloping ST depression during the exercise test, in the absence of chest pain. Patients were followed up for 12 months. Exercise tests were negative in 152 patients $(42 \%$ ) (group 1); silent ischaemia was observed in 103 patients $(29 \%)$ (group 2), and painful ischaemia observed in 82 patients $(23 \%)$ (group 3). The mortality in the first year was lowest in group $1(2 \%), 4 \%$ in group 2 , and $8 \%$ in group 3, with reinfarction rates of $3 \%, 8 \%$, and $18 \%$, respectively. The likelihood of subsequently developing angina was $16 \%$ (group 1 ) compared with $47 \%$ (group 2$),(p<0.001)$. These results indicate that silent ischaemia on exercise testing in survivors of acute myocardial infarction was common, being seen in $29 \%$ of patients.

Silent ischaemia defined in this way conferred an intermediate risk of subsequent cardiac events compared with those with a negative predischarge exercise test and those displaying painful ischaemia. In addition, silent ischaemia was a strong predictor of subsequent angina pectoris.

\section{Contraction/excitation feedback in man?}

P Taggart, $P$ M I Sutton, $T$ Treasure, $M J$ Lab, W O'Brien, R H Swanton, R W Emanuel Middlesex Hospital, London

Myocardial length/tension has been shown to influence repolarisation time and refractoriness in animals (contraction/excitation feedback) but, as yet, this has not been shown in man. During cardiopulmonary bypass the ventricle is in a relaxed, nonworking state with a systolic pressure of less than 50 $\mathrm{mm} \mathrm{Hg}$. Pressure is progressively increased over a period of about one minute as bypass is discontinued. A unique opportunity exists therefore to provide a clinical correlate for this feedback mechanism. Monophasic action potentials (MAPs) were recorded from the left ventricular epicardial surface as a measure of repolarisation time using a hand held pressure contact electrode. Arterial systolic pressure (radial cannula) was used as an indirect measure of LV systolic pressure. Measurements were made in 16 patients after routine coronary artery surgery. MAP duration shortened as systolic pressure increased. At $90 \%$ repolarisation MAP duration shortened from $288.0 \mathrm{~ms}$ (SD 29.5) at the onset of $\mathrm{LV}$ ejection on bypass to $261.0 \mathrm{~ms}(28.8)(\mathrm{p}<0.001)$ at completion of coming off bypass. Systolic pressure increased from $54.1 \mathrm{~mm} \mathrm{Hg}(9.3)$ on bypass to 75.5 $\mathrm{mm} \mathrm{Hg}(13.3)(\mathrm{p}<0.001)$ at completion. Regression analysis showed a strong correlation between MAP duration and systolic pressure $(p<0.001)$.

These results are consistent with the existence of contraction/excitation feedback in man. Such a mechanism may relate to the association of arrhythmias with asynergic wall motion by the creation of local inhomogeneous repolarisation times. 
Continuous electrocardiographic monitoring in male veteran endurance athletes

\section{R J Northcote, G J Canning, D Ballantyne The Victoria Infirmary, Glasgow}

The short term benefits of exercise are established. The long term effects of endurance exercise on cardiac function have not so far been assessed. Twenty male veteran endurance athletes were studied to establish the effect of chronic exercise and were compared with matched controls. The mean age of the athletes was 54 years, all had run at least 30 miles each week for at least 25 years. Forty eight hour continuous electrocardiography showed mean heart rate of $59 \mathrm{bpm}$ in athletes compared with $74 \mathrm{bpm}$ in controls $(p<0.001)$. Minimum heart rate in athletes was $37 \mathrm{bpm}$ compared to $47 \mathrm{bpm}$ in controls $(p<0.001)$. Circadian variation in heart rate was maintained. Fifteen out of 20 athletes had at least one ventricular extrasystole. Only four out of 20 had more than 50 extrasystoles per 24 hours. One athlete had in excess of 1600 extrasystoles in 24 hours, four athletes had complex forms. This was compared with 18 out of 20 controls who had PVCs, only four of whom had more than 50 in 24 hours but two of whom had evidence of SVT. There was no significant difference between the groups. Eight out of 20 athletes but only two out of 20 controls had periods of asystole ranging in length from 1.5-12 seconds, occurring in 846 occasions in the athletes and only four occasions in the controls. Two subjects had AV block, both of them exhibited first, second, and third degree heart block, and one required permanent pacemaker implantation.

Continuous running throughout life results in lower heart rates and greatly increased prevalence of asystolic pauses and AV node dysfunction. There is no difference in ectopic activity between athletes and controls.

\section{ECG analysis by computer and cardiologist: a multicentre cooperative study}

P W Macfarlane, J L Willems on behalf of the CSE Working Party

University Department of Medical Cardiology, Royal Infirmary, Glasgow, and Division of Medical Informatics, KUL, Leuven, Belgium

A large multicentre evaluation of the use of computers for ECG analysis has been in progress with the support of the European Economic Community (EEC) during the past few years. The pilot diagnos- tic phase of the study has now been reached whereby the accuracy of 11 programs (including many commercially available) and six cardiologists has been 0 assessed against a well defined clinical data base of 250 patients. The clinical diagnoses based on echocardiography and coronary arteriography, for example, consisted of seven mutually exclusive groups: normal, LVH, RVH, BVH, anterior, inferior, and combined myocardial infarction. Rhythm analysis and other ECG dependent diagnoses, (for example, $\overrightarrow{\vec{\omega}}$ conduction defects) were not included. The 250 o ECGs were analysed in different centres in Europe (including Glasgow), North America, and Japan. of The six European cardiologists interpreted the ECGs independently. All reports were sent to the of coordinating centre in Belgium for statistical analysis. The highest total accuracy of the computer programs was $76 \%$ (Glasgow Program) and the highest $\bar{c}$ total accuracy of an individual cardiologist was $78 \%$. There was a wide variation among programs and cardiologists not only in total accuracy but within individual diagnostic groups. BVH as well as multi- $\vec{\bullet}$ ple infarcts were analysed poorly by programs and $\infty$ cardiologists. The programs were superior in 0 detecting LVH but the cardiologists were better in reporting single infarcts. Predictive values were similar for programs and physicians.

The results of this preliminary study indicate that the Glasgow computer program is as accurate as cardiologists in interpreting an ECG when each is measured against clinical data.

\section{Computerised manikin for advanced resuscitation training}

R S Simons

Department of Anaesthesia, Royal Free Hospital, London

Advanced cardiac life support requires practical skills additional to basic cardiopulmonary resusci- o tation including the use of airway adjuncts, endo- $N$ tracheal intubation, arrhythmia recognition, N defibrillation, and intravenous access (peripheral $\omega$ and central). These activities need to be learned in an integrated and coordinated manner but no single manikin presently available can support this range of $\stackrel{\mathscr{D}}{\mathscr{D}}$ skills. A new computerised simulator capable of independent "life like" cardiorespiratory function is presented. The full length manikin supports basic $\mathbb{\mathbb { D }}$ and advanced cardiopulmonary resuscitation and is controlled by an Apple microcomputer which generates a range of ECG rhythms with appropriate stroke volume and blood pressure (BP) and palpable carotid 
pulse. Variable ventilatory rates and volumes are generated by a pump which provides realistic chest movements. Pupillary constriction and the achievement of an adequate supply of oxygen to the brain are indices of cerebral wellbeing. The ECG signal can be monitored from chest electrodes while a safe mock defibrillator with optional "quick look" paddles is used to teach defibrillation or synchronised countershock. Correct performance provides rhythm change and a realistic body jerk. One video display of graphics provides a two to three minute bar trend recording of $\mathrm{BP}$, respiratory volume, brain oxygen, and textual comment. A second page shows a display of the real time ECG and analogue waveforms.

This manikin permits team training under realistic conditions. It simulates a range of clinical problems and measures response and quantifies performance in an objective and practical manner.

\section{Evaluation of an advanced resuscitation training course for preregistration house officers}

W Kaye, Geralyn Wynne, Theresa Marteau, H G Dubin, Sharon Rallis, T R Evans

Cardiology Department, The Royal Free Hospital, London, Psychology Unit, Royal Free Hospital School of Medicine, London, and Brown University; The Miriam Hospital, Providence, Rhode Island and Centre for Evaluation and Research, Rhode Island College, Providence, Rhode Island, USA

Preregistration doctors in the "cardiac arrest" team need skills and knowledge of basic resuscitation and advanced cardiac life support (ACLS). A course was designed following the recommendations of the Resuscitation Council (UK) and a simplified version of the American Heart Association ACLS programme. The subjects were 60 newly qualified doctors who were assessed in two consecutive groups. As undergraduates they had been trained in basic resuscitation. Group $1(n=31)$ (starting their first job) were pretested and taught ACLS in two 2 hour sessions, and after five months retested to measure their skills. Group $2(n=29)$ had worked at other hospitals for six months but received no resuscitation training. They were taught and tested in the same way as group 1 at the beginning of their second post. At each session they completed questionnaires on their ability to perform resuscitation. In both groups the mean scores on knowledge and skill of resuscitation were significantly greater imme- diately after training. The knowledge and skills of group 1 five months after training were significantly greater than pretraining $(p<0.001)$. but slightly worse than immediately after training $(p<0.01)$. The more cardiac arrests the subjects attended, the more confident they felt in their ability to perform resuscitation; this was not matched by increasing competence. Five months after training there was a significant positive correlation between expressed confidence at performing resuscitation and demonstrated skill.

This study showed that the high degree of knowledge and skill acquired during the ACLS course were subject only to modest decay. Experience gave rise to false confidence and is not a substitute for training.

\section{Electrode pad size and defibrillation success}

G W N Dalzell, S R Cunningham, J Anderson, A A J Adgey

Regional Medical Cardiology Centre, Royal Victoria Hospital, Belfast

Although electrode size is an important determinant of current flow during countershock, larger pads have not been shown to be associated with increased defibrillation success in humans. To assess the effect of increasing the size of self adhesive ECG/ defibrillator pads on the success of defibrillation we studied 123 cardiac arrests (CA) due to primary ventricular fibrillation (PVF) in 105 patients (31 men, 74 women) aged 40-84 (mean 64 years). Transthoracic impedance (TTI) was measured before defibrillation using a $30 \mathrm{kHz}$ current passed through the chest via the ECG/defibrillator pads applied anteroanteriorly. We have previously shown that this method accurately predicts the actual impedance to a discharge. Pad diameters were small $(8 \mathrm{~cm} / 8 \mathrm{~cm})$ in $26 \mathrm{CA}$, intermediate $(8 \mathrm{~cm} / 12 \mathrm{~cm})$ in $63 \mathrm{CA}$, and large $(12 \mathrm{~cm} / 12 \mathrm{~cm})$ in $34 \mathrm{CA}$. Mean(SD) TTI decreased with increasing pad size $(112 \cdot 2 \Omega(17)$ vs $92 \cdot 3(22)$ and $71 \cdot 6(14)$, respectively, $(p=0.0001)$. Only the first episode of PVF during CA was analysed. A single shock of $200 \mathrm{~J}$ (delivered energy) was successful in 8 of $26(31 \%)$ CA with small pads, 40 of $63(63 \%)$ with intermediate pads, and 28 of 34 $(82 \%)$ with large pads, $p=0.0003$. A second $200 \mathrm{~J}$ shock increased the cumulative defibrillation rates to 12 of $26(46 \%), 50$ of $63(79 \%)$, and 33 of $34(97 \%)$ respectively, $\mathrm{p}<0.0001$.

In PVF larger self adhesive ECG/defibrillator pads are associated with reduced TTI and improved success rates for defibrillation with low energy shocks. 
112

\section{Patient delay in acute myocardial infarction}

J M Rawles, Neva Haites

Department of Medicine and Therapeutics, University of Aberdeen, Aberdeen

The longest component of the total delay in coming under coronary care is patient delay, and it has been suggested that public education might be successful in making it shorter. We have studied the patterns of patient delay and their association with late mortality in 450 patients with acute myocardial infarction uncomplicated by cardiac arrest, of whom 243 had a previous history of ischaemic heart disease. Patient delays showed a skewed distribution with a modal delay of up to 1 hour, a median delay of 2 hours, and a mean delay of 10 hours. Two thirds of patients had sought help within four hours of the onset of symptoms. During the first four hours the longer patients delayed the lower subsequent mortality $(27 \%, 18 \%$, and $9 \%$ for 0 to one, one to two, and over two to four hours, respectively). One explanation may be that those with larger infarcts call sooner. Patients who presented after four to eight hours, however, had the highest mortality of all $(38 \%)$, perhaps because such patients had developed serious complications of infarction. The pattern of patient delays was not altered by a previous history of ischaemic heart disease, with $69 \%$ and $68 \%$ of patients with and without a previous history of delaying up to four hours.

These findings suggest that the patient's call for help may be an instinctive pattern of behaviour which may therefore be difficult to modify by public education. Nevertheless a higher proportion of patients was seen early after infarction than in recent trials of thrombolytic treatment.

Direct admission to the coronary care unit with a telephone link system increases the number of patients suitable for thrombolytic treatment

Jennifer Burns, Kerry-Jane Hogg, A P Rae, W S Hillis, F G Dunn

Cardiology Department, Stobhill General Hospital, Glasgow

A narrow time window for administration of thrombolytic treatment in patients with acute myocardial infarction (AMI) makes it necessary to reduce to a minimum the delay in admission to the coronary care unit (CCU). While it is difficult to reduce the delay encountered in reporting of symptoms, avoidable delay has been identified in admitting the patients via
Proceedings of the British Cardiac Society

the casualty department and GPs have also commented on the delay in the response of hospital switchboards to incoming calls. We have therefore looked firstly at the effect of a direct "back door" admission policy to CCU in reducing the delay, and secondly at the combination of this admission policy plus the use of a telephone line which bypassed the hospital switchboard. A total of 95 patients were included in the study of whom $48 \%$ were subsequently shown to have had an AMI. In the first study the time from arrival of the GP at the patient's home until admission to the CCU was reduced from 88(30) minutes to $65(29)$ minutes $(p<0.05)$ and in the second study a direct admission policy plus telephone link reduced the delay from $90(51)$ minutes to $35(13)$ minutes $(\mathrm{p}<0.001)$.

These combined measures increased by twofold the number of patients admitted within a three hour time window from onset of symptoms and therefore increased the number of patients suitable for and likely to benefit from thrombolytic treatment.

Risk factors and in hospital outcome of a first myocardial infarction in the elderly

K Robinson, R Conroy, R Mulcahy

St Vincent's Hospital, Dublin, Ireland

The elderly form an increasingly large proportion of $\overrightarrow{\overrightarrow{0}}$ those admitted to the coronary care unit (CCU). Of a total of 980 patients, $53 \%$ were aged over 60 , and $24 \%$ over 70 years; they were studied to examine age-related differences in risk factors, in-hospital complications, and mortality. The proportion of females rose with age. The proportion of current $\vec{\sigma}$ smokers fell, while that of non-smokers rose, with age $(p<0.001)$. Age correlated negatively with total $\delta$ cholesterol and positively with high density lipo- $₹$ protein cholesterol $(\mathrm{p}<0.001)$. There was no cor- 은 relation between age and size or site of infarction. Complications, however, rose with age as did mortality, from $3 \%$ in the under-60s to $20 \%$ in those over $70(p<0.001)$. Cardiogenic shock and left ven- or tricular failure became significantly more lethal with $N$ rising age but ventricular fibrillation did not have a $\underset{\omega}{N}$ poorer outcome in older patients.

Coronary risk factors in the elderly differ significantly from those found in younger patients. $\stackrel{\overparen{D}}{\mathbb{D}}$ Complications and mortality increase in the elderly $\stackrel{\oplus}{-}$ following a first myocardial infarction, but ventricu- $T$ lar fibrillation is treatable in these patients who

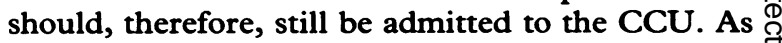
the coronary population ages, however, management $\mathbb{\mathbb { Q }}$ and secondary prevention methods derived from $\bar{O}$ studies confined to younger subjects may become inappropriate. 
Arrhythmia and prognosis in dilated cardiomyopathy

\author{
R A H Stewart, K Adams, W J McKenna, \\ Celia M Oakley \\ Department of Medicine (Clinical Cardiology), \\ Royal Postgraduate Medical School, Hammersmith \\ Hospital, London
}

The aim of this retrospective study was to assess the association between arrhythmia and prognosis in dilated cardiomyopathy (DCM). Follow up information has been obtained for 114 of 118 UK residents with DCM seen at this hospital since 1978. (74 men, 40 women, mean age 45). New York Heart Association (NYHA) class at diagnosis was class I in four; II in 90; III in 23, and IV in one patient. Ninety eight patients ( $86 \%$ ) had 24 hour ECG monitoring 30(33) months after diagnosis; mean(SD). Arrhythmia was frequent; AF in 16, SVT in 24, VT in 36 , couplets in 58 , and $>250$ ventricular extrasystoles/24 hours in 57 patients. Twenty three patients $(20 \%)$ have since died with disabling heart failure 48(40) months from diagnosis. Ten patients $(9 \%)$ died suddenly when heart failure was not disabling (NYHA class I, II, III). Five patients have received cardiac transplants. Four patients died from other causes. Fifty eight of the 72 patients alive on medical treatment have remained stable or improved 77(54) months after diagnosis. Of the patients who died, $78 \%$ had VT or $>250$ extrasystoles/24 hours compared to $67 \%$ of patients still alive (NS). Thirty two patients received amiodarone (median dose 200 $\mathrm{mg}$ /day) for AF/SVT in 14, symptomatic VT in five, or asymptomatic ventricular ectopy in 13. After a mean of 41(28) months on treatment there have been no sudden deaths. Three patients stopped amiodarone because of side effects (thyrotoxicosis, $\mathbf{n}=$ two, pulmonary fibrosis, $\mathrm{n}=$ one). There have been 10 sudden deaths in 82 patients who have not received amiodarone a mean of 57 months from assessment.

In conclusion, arrhythmia was unhelpful in predicting sudden death in DCM and a possible role for amiodarone in its prevention needs prospective evaluation.

\section{Mechanism of atrial natriuretic peptide release during tachycardia}

K P Walsh, T D M Williams, Charlotte Spiteri, Elaine Pitts, S L Lightman, R Sutton

Westminster Hospital, London

Atrial natriuretic peptide (ANP) concentrations are increased during paroxysmal tachycardia (PT) and also in conditions with increased atrial pressure. To see whether ANP release during PT is due to increased atrial rate or increased atrial pressure, we compared the ANP concentrations at different atrial pressures during identical tachycardia rates. We reduced atrial pressure during tachycardia by reducing venous return with balloon occlusion of the inferior vena cava (IVC). We compared the ANP responses to rapid atrial pacing over 50 beats per minute (bpm) increments from 200 to $350 \mathrm{bpm}$ with and without IVC occlusion in six chloralose anaesthetised dogs. During incremental pacing without IVC occlusion there was a marked rise in pulmonary wedge pressure (PWP)-mean (SEM)-from $5.3(1.6) \mathrm{mm} \mathrm{Hg}$ at $200 \mathrm{bpm}$ to $20.2(2.3) \mathrm{mm} \mathrm{Hg}$ at $350 \mathrm{bpm}(\mathrm{p}<0.01)$. At the same time, arterial and coronary sinus ANP concentrations rose from $116(55)$ and $339(91)$ to $1126(226)$ and $1960(456)$ $\mathrm{pmol} / \mathrm{l}$, respectively $(\mathrm{p}<0.01)$. In contrast, incremental pacing with IVC occlusion produced an insignificant increase in PWP from $-1.0(1.9)$ at 200 $\mathrm{bpm}$ to $2.3(1.8) \mathrm{mm} \mathrm{Hg}$ at $350 \mathrm{bpm}$. Arterial and coronary sinus ANP concentrations during IVC occlusion were respectively $208(126)$ and $388(159)$ at $200 \mathrm{bpm}$ and $261(83)$ and $345(80) \mathrm{pmol} / 1$ at 350 bpm (NS). A similar pattern was observed at intermediate pacing rates, with IVC occlusion preventing not only the rise in PWP but also the increase in plasma ANP concentrations with increasing heart rate.

The release of ANP during tachycardia is primarily dependent upon increased atrial pressure and not atrial rate.

\section{Diagnostic value of intravenous adenosine in patients with arrhythmias}

\section{A C Rankin, A P Rae, K G Oldroyd, E Chong, $S M$ Cobbe}

University Department of Medical Cardiology, Royal Infirmary, Glasgow

Establishing the correct diagnosis of arrhythmia has important therapeutic and prognostic implications, but is often difficult from the surface electrocardiogram. Clarification of the association between atrial and ventricular activity would aid diagnosis. Intravenous adenosine (dose range 2.5 to $20 \mathrm{mg}$, mean $7 \mathrm{mg}$ ) was given to 12 patients, seven men and five women aged 17-72 years (mean 52). All had paroxysmal tachycardias (rate 120-240 beats/minute, mean $174 \mathrm{bpm}$ ) with an apparently constant association between atrial and ventricular 
activity on their electrocardiograms. Adenosine clarified the diagnosis in all cases by its transient effect on atrioventricular (AV) nodal conduction. Supraventricular tachycardia with a re-entry mechanism involving the AV node was diagnosed in four patients in whom adenosine terminated the tachycardia by blocking AV conduction. In those patients with recurrent tachycardias, adenosine restored sinus rhythm in 14 of 15 episodes. Atrial tachycardia with $1: 1 \mathrm{AV}$ conduction was shown to be the diagnosis in two patients, and atrial flutter with $2: 1$ block in five. Adenosine produced a transient increase in AV block, slowing the ventricular response and revealing the atrial tachycardia. Ventricular tachycardia with 1:1 retrograde ventriculoatrial (VA) conduction was diagnosed in one patient in whom adenosine produced transient VA dissociation, with fusion and capture beats. Minor side effects, including flushing, chest discomfort, dyspnoea, and headache were common $(83 \%)$ but of short duration. No adverse haemodynamic response occurred.

Adenosine has diagnostic, in addition to therapeutic, value in patients with arrhythmias.

\section{Doppler diagnosis of broad complex tachycardias}

M J Griffith, D Mehta, A J Camm, D E Ward St George's Hospital Medical School, London

The differential diagnosis of broad complex tachycardia (QRS > $120 \mathrm{~ms}$ ) remains an important clinical problem. This study describes the use of ascending aorta Doppler velocimetry to quantify stroke volume to assess whether its variability may be used as a marker of independent atrial activity and thus identify ventricular tachycardia (VT).

Twenty patients with regular broad complex tachycardia were studied, 11 with VT and nine with supraventricular tachycardia (SVT). The diagnosis and AV relation were established by electrophysiology. Ascending aorta Doppler from the suprasternal notch was recorded using the Quinton Exerdop, a stand alone continuous wave Doppler. The maximum acceleration, peak velocity, and stroke distance were derived from 20 consecutive beats. There was no significant difference between the stroke distance, acceleration, or peak velocity for VT and SVT. The coefficient of variation was then calculated to assess if their variability could distinguish VT and SVT. Stroke distance was found to be the most discriminating, with a $20 \%$ coefficient of variation being $91 \%$ sensitive, and $100 \%$ specific, for ventricular tachycardia and $100 \%$ sensitive, and
$100 \%$ specific, for AV dissociation. The patient with VT and 1:1 retrograde conduction could not be distinguished from SVT. A coefficient of variation of $12 \%$ for peak velocity was $82 \%$ sensitive, $100 \%$ specific for VT, and $90 \%$ sensitive, $100 \%$ specific, for AV dissociation. A coefficient of variation of $25 \%$ for acceleration was $91 \%$ sensitive, $89 \%$ specific for VT and $100 \%$ sensitive, $90 \%$ specific, for AV dissociation.

This study shows that with Doppler imaging the variability of ascending aorta blood flow may be used to diagnose AV dissociation and VT in broad complex tachycardia. The simplicity of the technique should allow its use in the emergency setting.

Electrophysiological and antiarrhythmic effects of the acute class III actions of d-sotalol

J P Bourke, Janet McComb, J C Cowan, Suda Tansuphaswadikul, R W F Campbell

Freeman Hospital, Newcastle upon Tyne

The importance of acute class III effects in arrhythmia management is controversial. The clinical value and electrophysiological effects of d-sotalol were investigated in 10 patients (nine men; mean age 48 years) with life threatening ventricular arrhythmias (mean episodes 4.5 (range 3-10) and drug failures $2 \cdot 8$ ). Flecainide had failed in six and amiodarone in three. After baseline electrophysiology, $1-1.5 \mathrm{mg} / \mathrm{kg}$ of d-sotalol was infused over 10 minutes. Repeat electrophysiological study 20 minutes later showed significant changes in RR interval (786 (157) to 916 (175) ms; $p<0.05$ ), QT interval (368 (31) to 414 (46) $\mathrm{ms} ; \mathrm{p}<0.001$ ), right atrial effective refractory period (229 (46) to $271(38) \mathrm{ms} ; \mathrm{p}<0.025)$ and right ventricular effective refractory period (229 (22) to 267 (35) ms; p < 0.001). Right ventricular monophasic action potentials, measured in five of the 10 patients, increased from $310(55)$ to $335(61) \mathrm{ms}$ $(p=0.02)$. Ventricular arrhythmias were induced in all 10 patients before d-sotalol infusion and in eight afterwards (cycle length before was 257 (51) and after 273 (70) ms; $p=N S$ ). Ventricular arrhythmias did not recur on oral treatment in the two patients who were suppressed acutely. No arrhythmogenic effects were seen.

Despite clear evidence of acute class III effects, d-sotalol at this dosage had only modest success $(20 \%)$ in controlling life threatening ventricular arrhythmias. 
Accurate non-invasive prediction of arrhythmic events after acute myocardial infarction

T Cripps, A J Camm, E D Bennett, D E Ward Department of Cardiological Sciences and Medicine 1, St George's Hospital Medical School, London

Accurate prediction of arrhythmic events (sudden cardiac death and sustained ventricular tachycardia) after myocardial infarction is desirable both to reassure those at low risk and to test effectively the value of antiarrhythmic treatment in those at high risk. A prospective study was carried out in 134 consecutive patients admitted with acute myocardial infarction using high gain, signal averaged electrocardiography (SAECG) and 24 hour ambulatory electrocardiography (tape) within one week of infarction. Ten patients were not suitable for SAECG (bundle branch block in seven, atrial fibrillation in two, and complete heart block in one), and a tape result was not available in 22 (two refused, in 20 there were technical or administrative problems). The SAECG was abnormal in 33 of $124(27 \%)$; an ectopic frequency of $>5$ /hour was present in 26 of $112(23 \%)$. There was both an abnormal SAECG and $>5$ extrasystoles per hour in nine of $107(8 \%)$. During two to 12 months follow up, 13 patients $(10 \%)$ suffered an arrhythmic event (documented sustained ventricular tachycardia (VT) in five, suspected VT in one, and sudden cardiac death without apparent reinfarction in seven). Discriminant analysis showed the tests to have the following ability in the prediction of arrhythmic events-SAECG: sensitivity 85\%, specificity $82 \%$, predictive accuracy $33 \%$; tape: sensitivity $88 \%$, specificity $82 \%$, predictive accuracy $27 \%$; SAECG and tape: sensitivity $85 \%$, specificity $98 \%$, predictive accuracy $78 \%$.

Though each of these tests used alone lacks specificity, the combination of the two appears to be accurate enough to be of clinical value in assessing arrhythmic propensity in patients after myocardial infarction.

\section{Evolving indications for cardiac transplantation}

M Jones, A H Menkis, W J Kostuk, F N McKenzie University Hospital, London, Ontario, Canada

Between April 1981 and January 1987100 cardiac transplants were performed in 96 patients $(70$ men, 26 women). Sixty seven patients were less than 50 years old, and 29 ranged in age from 50 to 64 years.
Recipient diagnosis was similar in both age groupscardiomyopathy $56 \%$, coronary artery disease $34 \%$, valvar $6 \%$, and graft failure $4 \%$. The one year survival has improved from $70 \%$ for the first 50 to $81 \%$ for the second 50 . The majority of deaths (20) occurred within 90 days of surgery. Only one death was due to infection and there were only four graft losses (one successful retransplantation) due to rejection. Although one year survival of $86 \%$ is higher for patients over 50 years old compared with $69 \%$ for patients less than 50, the former group comprises most of the last 50. Functional status and cardiac function has improved from New York Heart Association class IV and left ventricular ejection fraction mean $15 \%$, to class I and class $\mathrm{II}$ and ejection fraction mean $60 \%$. Forty patients have had one or more coronary angiograms performed one to five years after transplantation. No patient has shown any segmental lesion(s) on follow up for 12 to 72 months (mean 28 months). All patients received aspirin treatment. The improved results are reflected in broadening donor and recipient criteria; we accept donor dopamine support of up to $15 \mu \mathrm{g} / \mathrm{kg} / \mathrm{min}$ and a flight time of up to three hours.

The presence of pulmonary embolism, diabetes, treated infection, and the presence of peripheral or cerebral vascular disease are not contraindications to transplantation.

\section{Coronary occlusive disease in the transplanted heart}

H Davies, G I Verney, J Wallwork, T A H English Papworth Hospital, Cambridge

Accelerated coronary occlusive disease in the donor heart is an important determinant of survival after cardiac transplantation. Although the aetiology remains obscure, the initiating injury to the endothelium is probably immunologically induced. This presents early as diffuse narrowing of the small and medium sized vessels whereas later individual risk factors may contribute to progression of segmental disease affecting mainly the larger vessels. Between January 1979 and June 1985, 117 patients received orthotopic heart transplants. Initially all patients had coronary angiograms annually. More recently, the first angiogram has been carried out at two years. If normal, the next examination is at four years; if abnormal, at three years. Thereafter annual angiograms are performed. Eighty two of the 117 patients survived two or more years after transplantation $(70 \%)$ and 208 angiograms have been performed between one and seven years after their transplant. 


\section{6}

Angiograms have been graded according to a scale $0=$ apparently normal; I = slight irregularity; II = definite change but less than $50 \%$ narrowing in one or more vessels; III = severe changes of more than $50 \%$ narrowing in diameter. Angiographic disease was evident in $18 \%$ and $25 \%$ of patients at two and five years, respectively.

There was wide variation in the time of onset, severity, and rate of progression. Three patients have had further transplants and nine have died, of whom three had necropsy evidence of occlusive disease within a year of an apparently normal angiogram. In addition, four patients, who died from rejection between six and 12 months after transplantation, showed marked narrowing of their coronary vessels due to diffuse myointimal proliferation.

Isometric exercise in the denervated human heart: a Doppler echocardiographic study

\section{S C Robson, S S Furniss, A Heads, R J Boys, R S Bexton \\ Department of Cardiology, Freeman Hospital, Newcastle upon Tyne}

The haemodynamic response of the denervated heart to isometric exercise has not to our knowledge been previously reported. Eight orthotopic heart transplant recipients (age 40 (10) years) and eight healthy controls (age 25 (7) years), age and sex matched to the donor hearts, were studied. Using a hand grip dynamometer each subject performed sustained exercise at $30 \%$ of maximal voluntary contraction for a period of three minutes. Cardiac output was measured using combined Doppler and cross sectional echocardiography before exercise, at $30 \mathrm{sec}-$ ond intervals during exercise, and after exercise. Aortic velocities were recorded using continuous wave Doppler from the suprasternal notch. Simultaneous cross sectional echocardiographs of the aortic orifice and left ventricular $M$ mode echocardiographs were recorded. In the control group cardiac output increased from 6.11 /minute at rest to $8.11 /$ minute after three minutes exercise $(p<0.001)$, due to an increase in heart rate from 70 to 92 beats/minute $(p<0.001)$ with no change in stroke volume. There was no significant change in left ventricular end diastolic and end systolic dimensions or ejection fraction. In the transplant group cardiac output, heart rate, and stroke volume remained unchanged throughout the period of exercise. There was, however, a small decrease in ejection fraction. In both groups there was an increase in stroke volume immediately after exercise $(p<0.01)$.

\section{Proceedings of the British Cardiac Society}

In contrast to dynamic exercise, the denervated human heart is unable to increase cardiac output during isometric exercise, and Doppler echocardiography is a useful technique for the noninvasive measurement of haemodynamic changes during isometric exercise.

\section{Prevalence and significance of coronary artery disease after cardiac transplantation in patients receiving cyclosporin and azathioprine}

C J Reid, N Banner, C O'Brien, A Tizard, A G Mitchell, $M$ H Yacoub

Harefield Hospital, Middlesex

Coronary artery disease (CAD) is a potentially serious late complication of cardiac transplantation. Its prevalence, influence on exercise capacity, left ventricular function, and late mortality have been examined in this study. Three hundred and sixty two patients underwent orthotopic cardiac transplantation between September 1982 and July 1987 and received cyclosporin (CYA) and azathioprine (AZA). The cumulative probability of development of $\mathrm{CAD}$ is $7 \cdot 2,12.5$, and $18.5 \%$ at one, two, and three years after operation, significantly different from a group of historical controls who had received prednisolone and AZA and who had a cumulative probability of development of CAD of 15,33 , and $96 \%$, respectively. All patients with CAD are in New York Heart Association Class I or II. In a specific group of patients investigated three years after transplantation, the duration of treadmill exercise in patients with CAD was $9 \cdot 9(0 \cdot 84)$ minutes and with normal coronary arteries (NCA) $11.6(0.49)$ minutes $(p<0.001)$. Left ventricular ejection fraction, determined by radionuclide angiography at rest, was $65.0(4.8)$ in CAD and $69.0(1.7)$ in NCA $(\mathrm{p}<0.05)$, and on exercise $73.3(4 \cdot 8)$ and $74.1(1.6)$ (NS), respectively. The late ( $>1$ year) mortality in patients with CAD was two of $22(9 \cdot 1 \%)$ in a followup of one to 37 (mean 10.9) months. These deaths, however, constitute $67 \%$ of the mortality of all patients during this period.

This study shows that the prevalence of coronary artery disease in patients receiving double or triple treatment including cyclosporin $\mathrm{A}$ is significantly lower than conventional immunosuppression and that this complication carries a guarded though not serious prognosis in the medium term. 


\section{Diurnal variation in ECG QRS voltage}

\author{
G Parry, Janet McComb, R W F Campbell \\ Freeman Hospital, Newcastle upon Tyne
}

A decrease of $>20 \%$ in summated peak to peak $Q R S$ voltage in leads I, II, III, V1, and V6 in cardiac transplant recipients has been associated with rejection. Although ECG voltage measurements are known to vary from day to day the possibility of physiological diurnal variation in $Q R S$ voltage has not to our knowledge been investigated. QRS voltage, duration and frontal axis, QTc interval, and heart rate were studied during 24 hours in 16 normal subjects. ECGs were obtained at $7 \mathrm{pm}, 11 \mathrm{pm}$, and 7am. Electrode site, subject position, and ECG recorder were constant. Summated QRS voltage was lower at $7 \mathrm{am}$ (mean (SD) $4.65(0.88) \mathrm{mV}$ ) than at $7 \mathrm{pm}(5.37(1.04), \mathrm{p}<0.001)$, or at $11 \mathrm{pm}(5.34(1.04)$, $\mathrm{p}<0.001)$. This $\mathrm{QRS}$ voltage decrease was $>20 \%$ in four of 16 subjects. Heart rate was higher at $7 \mathrm{pm}$ $(74.9(12.9) \mathrm{bpm})$ than at either $11 \mathrm{pm}(68.8(11.6)$, $\mathrm{p}<0.02)$ or $7 \mathrm{am}(68.7(10.5), \mathrm{p}<0.05)$. Change in QRS voltage did not correlate with heart rate change. QTc was shorter at 7am $(387 \cdot 2(22 \cdot 4) \mathrm{ms})$ than at $7 \mathrm{pm}(398.6(27.4), \mathrm{p}<0.05)$. QRS duration and axis did not change.

There is therefore a substantial diurnal variation in QRS summated voltage in normal subjects. Similar diurnal voltage changes in patients would have implications for the diagnosis of cardiac rejection and for assessment of infarct size and left ventricular hypertrophy by voltage measurement.

\section{Why do angiotensin converting enzyme inhibitors affect mortality in chronic heart failure? Effects on $\beta$ adrenergic receptor function}

N Anfilogoff, R J Bain, N Lawson, Amanda Sadler, R G Murray, W A Littler, M K Davies

Department of Cardiovascular Medicine, East Birmingham Hospital, Birmingham

The mechanism of the beneficial effects of angiotensin converting enzyme (ACE) inhibitors, as shown by the reduction in mortality from chronic heart failure (CHF) in the CONSENSUS study, is unknown. In $\mathrm{CHF}$ it has been shown that down regulation of $\beta$ adrenergic function occurs due to chronic sympathetic nervous system overstimulation and that ACE inhibitor treatment reduces raised circulating catecholamine concentrations. An indirect assessment of $\beta$ adrenergic receptor function may be made, in man, by assessing circulating lymphocyte $\beta$ adrenergic receptor responsiveness (LBRR) in vitro. In this study the effect of captopril on LBRR was examined prospectively in eight patients with CHF. (five-New York Heart Association class III; threeclass IV; mean age 60.2 (6.4) years; mean ejection fraction by cross sectional echocardiography $22 \cdot 3$ $(4 \cdot 1) \%$. LBRR was determined by measuring increases in intracellular cyclic AMP in response to stimulation, in vitro, by incremental concentrations of isoprenaline (IP) $\left(10^{-3} \mathrm{mmol} / 1-50 \mathrm{mmol} / \mathrm{l}\right)$. Results, expressed as stimulated increase in cyclic AMP over basal measurements, showed increased responsiveness after six weeks treatment with captopril, across the concentration range. The results were significantly different at the $10^{-3} \mathrm{mmol} / 1$ and $1 \mathrm{mmol} / 1$ IP concentrations (112 $(5.2) \%$ and $147(10.2) \%$ respectively, (p < 0.025); $122(13.9) \%$ and $151(7 \cdot 7) \%$, respectively, $(\mathrm{p}<0.05)$; mean $(\mathrm{SEM})$ before and after treatment, respectively).

Severe CHF results in the down regulation of the $\beta$ adrenergic receptor population. These data suggest that treatment with captopril has improved $\beta$ adrenergic receptor function and may in part explain the known clinical benefit of ACE inhibitors.

\section{Balloon dilatation of calcific aortic stenosis}

G Jackson, D Sprigings, S Thomas, M Monaghan, J Chambers, D Jewitt

Cardiac Department, King's College Hospital, London

The place of balloon dilatation (BD) in the management of calcific aortic stenosis remains controversial. We have performed BD in 27 elderly patients (mean age 77, range 65-86) in whom valve replacement was contraindicated. Five patients were in the New York Heart Association (NYHA) class II, 15 in class III, and seven in class IV. Tamponade occurred in one patient after perforation of the ventricle by a guidewire. There have been no other major complications and no clinically apparent embolic events. Mean peak to peak aortic valve gradient fell from $66 \mathrm{~mm} \mathrm{Hg}$ (range 20-116) to $46 \mathrm{~mm} \mathrm{Hg}$ (range 0-92) immediately after BD. Mean aortic valve area (calculated by the modified continuity equation from Doppler velocity measurements before and one to seven days after BD) showed a slight increase from $0.5 \mathrm{~cm}^{2}$ (range $0.2-0.9$ ) to $0.6 \mathrm{~cm}^{2}$ (range $0.2-1 \cdot 1$ ). Seventeen patients $(63 \%)$ reported an improvement in symptoms after BD. Of 19 survivors, four are in NYHA class I, nine in class II and six in class III. 
Eight patients (all in class III or IV before BD) have died, three of non-cardiac disease. In stenotic valves without commissural fusion BD may achieve only a small or temporary increase in valve area by stretching non-calcified tissue in the valve ring or rendering the cusps more pliable.

In elderly patients with calcific aortic stenosis, BD can provide palliation of symptoms with a low incidence of complications. The mean increase in valve area, however, is small and it cannot be regarded as an alternative to valve replacement in surgical candidates.

\section{Follow up of patients after balloon dilatation of the mitral valve}

T R D Shaw, C M Turnbull, P Bloomfield, B Saeed, A T Elder

Department of Cardiology, Western General Hospital, Edinburgh

Of 11 patients undergoing dilatation of the mitral valve by a $25 \mathrm{~mm}$ diameter balloon, nine have been restudied one to four months later: one patient had died from respiratory disease shortly after dilatation and in one patient the balloon catheters could not be made to cross the resistant interatrial septum. At follow up cardiac catheterisation of these nine patients the mitral valve gradient had been reduced from 10.7 to $6.6 \mathrm{~mm} \mathrm{Hg}(-38 \%)$ and resting cardiac output had risen from 3.37 to 4.041 /minute $(+20 \%):$ the mitral valve area had increased from $0.60 \mathrm{~cm}^{2}$ to $1.30 \mathrm{~cm}^{2}(+46 \%)$. There was no correlation between haemodynamic improvement at rest and the extent of mitral valve calcification. Before balloon dilatation four of the 11 patients had been unable to leave hospital because of breathlessness; all had associated severe medical problems in addition to their mitral stenosis and were considered unsuitable for or at high risk of operation. One of these patients died, but three were symptomatically improved and able to be discharged from hospital. Four of the other six treated patients showed symptomatic improvement; the remaining two patients had a moderate degree of mitral stenosis and were either unsuitable for balloon dilatation or required the larger $30 \mathrm{~mm}$ diameter balloon. Improvement was also seen in the echocardiographic features of the mitral valve disease.

Balloon dilatation of the mitral valve is an important and cost effective procedure for high risk surgical candidates and a useful alternative to mitral valvotomy in all patients with mitral stenosis.
Balloon dilatation of the pulmonary valve in neonates: technical considerations and results

J A Ettedgui, R P Martin, O D H Jones, E J Baker, J F Reidy, M J Tynan

Guy's Hospital, London

Balloon dilatation of a severely stenosed pulmonary valve (PV) was attempted in seven neonates. In three, the diagnosis had been made prenatally. Various technical difficulties were encountered at the time of cardiac catheterisation. In all of them the right ventricular (RV) pressure was at systemic or suprasystemic height. The arterial duct was widely patent in five. In one of these five it was not possible to cross the PV and he was referred for a surgical valvotomy. In two patients, placing the guide wire in a safe position in the pulmonary artery was time consuming and difficult. In the remaining two it was not possible to enter the branch pulmonary arteries and balloon dilatation was safely performed with the balloon catheter over a guide wire anchored in the descending aorta through the duct. In these patients it was difficult to judge initial success because of the presence of the widely patent duct. In the two without an arterial duct the balloon dilatation was performed by a standard technique. In one neonate balloon dilatation was performed as a staged procedure. At age 6 hours only a $3 \mathrm{~mm}$ coronary angioplasty balloon could be manipulated across the pulmonary valve. Repeat BD three weeks later with an $8 \mathrm{~mm}$ balloon reduced the RV pressure from $85 \mathrm{~mm} \mathrm{Hg}$ to $30 \mathrm{~mm} \mathrm{Hg}$. Follow up cardiac catheterisation has been performed in three infants. Balloon dilatation failed in two, the RV pressure being at systemic height. One of them had a dysplastic PV. In the other, failure may be due to inappropriate balloon size; repeat dilatation was precluded by inferior vena cava thrombosis. Both have been referred for a surgical valvotomy. A good result has been confirmed in the remaining two children.

We conclude that balloon dilatation of the pulmonary valve in neonates is technically difficult and may not produce uniformly good results.

Value of Doppler echocardiography in balloon dilatation of aortic stenosis

P Nihoyannopoulos, J Crick, G Karatasakis, Celia M Oakley

Royal Postgraduate Medical School, Hammersmith Hospital, London

To assess the role of echocardiography in the promising new treatment method of balloon dilatation of 
aortic stenosis we used cross sectional echocardiography, Doppler, and colour flow imaging before, during, and after anterograde balloon dilatation in five patients, 76 to 87 years of age (mean 82). During the procedure cross sectional echocardiography was used to guide transseptal catheterisation and to show appropriate balloon positioning across the stenotic valve. Aortic valve morphology, left ventricular fractional shortening, peak gradients (Doppler) and the presence of aortic incompetence were assessed immediately before, one day, and one month after balloon dilatation. Following balloon dilatation the peak to peak catheter gradients dropped from 74 (24) to 42 (22) $\mathrm{mm} \mathrm{Hg}$ (p < 0.001). Peak instantaneous gradients dropped from 87 (20) to 55 (21) $\mathrm{mm} \mathrm{Hg}(p<0.001)$. There was slight increase in aortic cusp separation on echocardiography in 45 patients though the fractional shortening remained unchanged. The valve morphology on the initial study was not useful in predicting the patient's outcome. All patients had only mild aortic incompetence and none developed any detectable change on colour flow mapping following the procedure. A small degree of left atrial to right atrial shunt was seen immediately after the transseptal procedure; this was not, however, seen subsequently. Repeat Doppler studies after one month showed no change in three patients, a $10 \%$ return towards the initial gradient in one, and a $31 \%$ return in another patient, who subsequently died.

We conclude that modern Doppler echocardiography helps the performance of balloon dilatation and is an invaluable tool for the long term follow up.

\section{Park blade septostomy: results and selection of patients}

J V De Giovanni, E D Silove

Department of Cardiology, Children's Hospital, Birmingham

The technique of atrial septostomy using a blade tipped catheter has been used in our department in the past five years when balloon atrial septostomy has failed to achieve good atrial mixing, or when the septum is considered too tough for ballooning. Between 1982 and 1987 twenty blade septostomies were performed on 19 patients. Their ages ranged from 23 days to 8.5 years (mean 429 days). Eight had discordant ventriculoarterial connections as the main abnormality, six of whom had previous balloon septostomy (group 1). Eleven had right or left atrioventricular valve atresia (group 2). In group 1 the atrial gradient dropped from 5.7 to $3.2 \mathrm{~mm} \mathrm{Hg}$, whereas in group 2 it fell from 6.3 to $2.5 \mathrm{~mm} \mathrm{Hg}$ after blade septostomy (NS). The oxygen saturation in group 1 rose in five patients. Four patients in group 1 , and five patients in group 2, showed clinical improvement after the procedure. There were no major complications.

Blade atrial septostomy is effective in patients with restrictive atrial communication especially in the older infant and young child and in the presence of atrioventricular valve atresia. Neonates with discordant ventriculoarterial connections who fail to improve with balloon septostomy, however, are unlikely to benefit from this technique especially when dynamic left ventricular outflow obstruction contributes more to the cyanosis than inadequate atrial mixing.

\section{Percutaneous balloon dilatation for infant aortic valve stenosis}

C Wren, I D Sullivan, Catherine Bull, J E Deanfield Freeman Hospital, Newcastle upon Tyne, and Hospitals for Sick Children, Great Ormond Street, London

Percutaneous balloon dilatation was attempted in 13 consecutive infants with critical aortic valve stenosis. Their ages ranged from 1 day to 11 months (median 4 weeks) and their weights from 1.5 to $8.9 \mathrm{~kg}$. Ten patients were in heart failure. Three had already undergone surgical valvotomy. The balloons were 5-12 $\mathrm{mm}$ in diameter (at least 1-2 mm smaller than the aortic valve measured on an aortogram). In two patients the valve could not be crossed and both died during subsequent operations. Another two patients died during manipulation of the balloon catheter. No benefit was obtained in a further patient who also died later during operation. Balloon dilatation was successful in the remaining eight patients, reducing their aortic valve pressure gradients from 65 (25) to 23 (16) $\mathrm{mm} \mathrm{Hg}$; mean (95\% confidence intervals). Two of these eight patients subsequently died from heart failure related to other cardiac lesions. The six survivors have done well. Two required femoral artery thrombectomy. None developed appreciable aortic regurgitation. At follow up the maximum ascending aortic Doppler velocities ranged from 1.9 to $4.0 \mathrm{~m} / \mathrm{s}$ after two to 23 months but, despite this evidence of increasing valve gradient, there has been lasting ctinical improvement. None of the five patients weighing less than $3 \mathrm{~kg}$ survived long term, whereas six of eight patients heavier than $3 \mathrm{~kg}$ are alive and well. 
Although an improvement in the results of percutaneous balloon dilatation can be expected with increasing practical experience and improved balloon catheter design, relief of aortic valve stenosis in patients weighing less than $3 \mathrm{~kg}$ is likely to remain difficult whether performed surgically or by balloon catheter.

\section{Results of the first 200 percutaneous transluminal coronary angioplasties in a regional cardiac unit}

\section{P J B Hubner, J K Walker, C Cook, S Madden Groby Road Hospital, Leicester}

Between December 1982 and December 1986, 200 percutaneous transluminal coronary angioplasties (PTCA) were performed by a single operator. Two hundred and seven vessels were dilated in 170 patients. The overall primary success rate was $73 \%$. There was a learning curve with the success rate rising from $68 \%$ to $88 \%$ from the first 50 cases to the last 50 cases. Vessel occlusion occurred in 16 patients $(8 \%)$ and was usually managed conservatively so that only five cases $(2.5 \%)$ were sent for urgent coronary artery bypass grafting (CABG). The incidence of myocardial infarction $(80 \%)$ was not reduced by urgent CABG. There were two deaths $(1 \%)$ in the series, one at the time of emergency CABG, the other during PTCA for an elderly patient with multivessel disease and poor left ventricular function. The symptomatic restenosis rate was $22 \%$. PTCA procedures have been carefully costed at $£ 1390$, comparing favourably with $£ 3638$ for CABG in this unit.

These results have given us confidence to attempt the more complicated cases with multivessel disease that have formed $42 \%$ of the last 50 cases of the series.

\section{Effect of change in reference diameter on percentage diameter stenosis measurement during and after coronary angioplasty}

K J Beatt, $H$ E Luijten, $H \mathrm{~J}$ ten Katen, H Suryapranata, P W Serruys, P G Hugenholtz Thoraxcenter, Rotterdam, The Netherlands

Conventional assessment of coronary angioplasty (PTCA) is often based on the changes in percentage diameter stenosis of the dilated lesion. This mea- surement is dependent on two variables, the reference diameter (diameter of nearest normal segment) and the minimal lumen diameter. We investigated the changes in these two variables in 342 patients with 398 lesions who underwent successful PTCA. Patients were reinvestigated after angioplasty at one of four predetermined follow up times: 30 days, 60 days, 90 days, or 120 days. Measurements were performed using a quantitative automated edge detection system (CAAS). Following the PTCA the mean reference diameter was $2.93(0.55) \mathrm{mm}$ and the minimal lumen diameter $2.08(0.43) \mathrm{mm}$, with no differences among the four groups. $(p=0 \cdot 17$, and $p=0 \cdot 34$, respectively). At follow up in the 30 and 60 day groups there were small non-significant changes in both the reference diameter and the minimal lumen diameter. At 90 and 120 days, however, there were highly significant reductions $(p<0.0001)$ in both the reference diameters, $-0.17(0.41) \mathrm{mm}$ and $-0.26(0.44) \mathrm{mm}$, respectively, and the minimal lumen diameters $-0.37(0.54) \mathrm{mm}$ and -0.42 $(0.52) \mathrm{mm}$, respectively. Restenosis following angioplasty takes place predominantly after the first 60 days after angioplasty with evidence of continuing deterioration up to 120 days. The reference diameter also deteriorates at 90 and 120 days.

The significant change in the reference diameter will lead to an underestimation of the change in a dilated lesion if assessment of the change is based on percentage diameter stenosis alone.

Incidence, importance, and determinants of aortic regurgitation after anatomical correction of transposition of the great arteries

R P Martin, E J Ladusans, J M Parsons, E Keck, R Radley-Smith, M H Yacoub Harefield Hospital, Middlesex

To determine the incidence and severity of aortic regurgitation (AR) after anatomical correction of transposition of the great arteries, the postoperative aortic root angiograms of 55 children were evaluated. Angiograms were performed from four to 56 (mean 15.6) months after operation. AR was present in 25 patients $(45 \%)$. This was trivial or mild in 24 patients $(96 \%)$ as defined by the presence of an aortic regurgitant jet without pronounced opacification of the left ventricle. In one patient there was grade 3 AR (the only patient with an aortic regurgitant murmur). AR was present in 16 of 26 patients $(62 \%)$ that had undergone preliminary pulmonary artery banding as part of a two stage corrective operation: three 
of eight patients $(37 \%)$ that had primary correction with closure of ventricular defect, and two of 17 patients $(12 \%)$ that had primary correction as a neonate. Measurements of the aortic root diameter at the level of the aortic commissures were significantly $(p<0.05)$ higher than normal values. Commissural diameter expressed as \% of normal value (1SD) was $146(24) \%$ after two stage correction; $133(21) \%$ after primary correction with closure of ventricular septal defect, and $119(19) \%$ after primary correction as a neonate, suggesting that an increased commissural diameter is an important determinant of AR. The method of coronary artery transfer and presence of aortic anastamotic narrowing (present in four patients $(6 \%)$ ) did not influence the incidence of AR.

Trivial AR is common after anatomical correction and may be related to an increased diameter of the aortic root at commissural level. There has been no clinical evidence of progression of the degree of AR during the medium term; its long term importance, however, remains undetermined.

\section{Complete repair of common arterial trunk}

I Th Fessatidis, K E Vassiliadis, B R Keeton, G R Sutherland, J L Monro

Wessex Cardiothoracic Centre, General Hospital, Southampton

From July 1974 to March 1987, 15 infants with common arterial trunk (14 type 1, one type 2 ) underwent complete repair, five cases $(33.3 \%)$ as emergencies, at the Wessex cardiac surgery centre. At operation, mean age was 12 weeks (range 5 days -10 months) and weight $4 \cdot 1 \mathrm{~kg}$ (range $2 \cdot 5-7 \cdot 5 \mathrm{~kg}$ ). Surface cooling and profound hypothermic circulatory arrest were used in all cases with a mean arrest time of $63.46 \mathrm{~min}$ (range 47-86 minutes). Aortic homografts were used as the conduit, mean size $16.3 \mathrm{~mm}$ (range $15-18 \mathrm{~mm})$. Three patients died postoperatively $(25 \%)$, two of whom were emergencies. There were no late deaths. Mean follow up is $\mathbf{5 . 9}$ years (range 3 months-13 years). Eight patients survived a cardiac arrest in the early postoperative period of whom one has permanent cerebral damage. One patient who had truncal valve regurgitation preoperatively required aortic valve replacement four years postoperatively and the conduit was replaced at the same time. All survivors are well and recent Doppler echocardiography shows a mild gradient across the conduit varying from $16-36 \mathrm{~mm} \mathrm{Hg}$ except in one recent patient weighing $2.5 \mathrm{~kg}$ who has a conduit gradient of $80 \mathrm{~mm} \mathrm{Hg}$ and will require reoperation.
Complete repair of common arterial trunk in early infancy continues to give good clinical and haemodynamic results more than 13 years after operation.

\section{Pulmonary atresia with ventricular septal defect and multifocal pulmonary blood supply: results of a policy of surgical unifocalisation}

I D Sullivan, C Wren, F J Macartney, J Stark, $M$ de Leval, J E Deanfield

Hospitals for Sick Children, Great Ormond Street, London

Single stage repair is not possible in patients with pulmonary atresia and ventricular septal defect, multifocal blood supply, and small central pulmonary arteries. Between 1979 and 1986 unifocalisation surgery, aimed at reducing sources of pulmonary blood supply by connecting major aortopulmonary collateral arteries (MAPCAs) to central pulmonary arteries without sacrificing segmental perfusion, was performed in 26 patients with 3.9(1.1) MAPCAs per patient. The aims were to provide palliation and prepare the circulation for definitive repair. Age at first operation was 2 weeks13 years, median 2 years, with eight being less than 1 year old. There were four deaths $(15 \%)$. The number of bronchopulmonary segments connected to the central pulmonary artery increased from $5 \cdot 5(4 \cdot 9)$ to $8.7(5.4)(p<0.001)$ and central pulmonary artery growth occurred (RPA + LPA/descending aorta ratio increased from $0.64(0.61)$ to $0.95(0.60)$, $\mathrm{p}<0.01)$. This was accompanied by improvement in symptoms and systemic oxygen saturation. At restudy systemic artery to central pulmonary artery grafts had a better patency rate than did grafts or anastomoses involving MAPCAs (21 of 22 and 20 of 33 , respectively, $p=0.003$ ). Subsequent repair was performed in two $(8 \%)$ patients with one death, and only four $(15 \%)$ others are considered correctable.

Unifocalisation provided reasonable palliation at the cost of appreciable mortality. The increases in central pulmonary artery size and connection obtained in these complex young patients were modest and rarely permitted complete repair.

\section{Waterston shunt revisited}

J R L Hamilton, N Wilson, D F Dickinson, D R Walker

Killingbeck Hospital, Leeds 
In recent times the modified Blalock-Taussig operation has become the systemic pulmonary shunt of choice in most centres and the Waterston anastomosis has fallen into disuse. In this unit, however, the Waterston shunt has continued to be the first shunt of choice particularly in small infants, and this paper presents our experience over a seven and a half year period from 1979-1986. Included are 74 consecutive infants ranging in age from 1 day to 20 months (median 5 weeks) whose weights ranged from $1.8-9 \mathrm{~kg}$ (median $3.6 \mathrm{~kg}$ ). The major diagnostic categories were tetralogy of Fallot $(31 \%)$, pulmonary atresia and ventricular septal defect $(21 \%)$, pulmonary atresia and intact ventricular septum $(17 \%)$, tricuspid atresia $(5 \%)$, and the remainder comprised a heterogeneous group of complex lesions with reduced pulmonary blood flow. There were six operative deaths (mortality $8 \%$ ), and four infants required reoperation for early shunt failure one day to three weeks postoperatively. The maximum follow up is 92 months (average 25 months) and there have been 11 late deaths, none related to shunt failure. Corrective surgery has been performed in eight children at a median period postoperatively of 46 months and seven further children are due to be corrected in the near future. Seven of the 68 children discharged from hospital have required a second shunt at a median of 31 months postoperatively.

We believe these results support the continuing use of the Waterston anastomosis, particularly in small infants.

\section{Fate of children with tricuspid atresia presenting in infancy}

R C G Franklin, I D Sullivan, D E Thoele, F J Macartney, R H Anderson, J E Deanfield Hospitals for Sick Children, and the Brompton Hospital, London

We reviewed the clinical outcome of 225 patients with tricuspid atresia who presented within the first year of life between 1972 and 1987. Ventriculoarterial connection was concordant (CONC) in $151(67 \%)$, discordant (DISC) in $58(26 \%)$, and neither in $16(7 \%)$. There was evidence of systemic outflow obstruction at presentation in $34(54 \%)$ of patients with DISC (subaortic stenosis in nine, coarctation in 13, both in 12). Cox regression identified systemic outflow obstruction, ECG left ventricular strain, and presentation at a younger age or without cyanosis as independent adverse risk factors for survival. The actuarial survival was similar for patients who underwent a systemic to pulmonary $\overrightarrow{\vec{D}}$ artery shunt and for those who had pulmonary artery banding alone $(79 \%$ and $81 \%$ at one year, and $66 \%$ and $63 \%$ at five years, respectively). Only two of $25(5 \%)$ patients with coarctation survived beyond one year of age, despite surgery in 17 of $25(68 \%)$. As a result, patients with DISC had a five year actuarial in survival significantly worse than patients with $\vec{\circ}$ CONC $(34 \%$ and $61 \%$, respectively, $\mathrm{p}=0.0001)$. There were 82 patients with CONC and 18 patients with DISC who survived beyond two years of age. Of these patients, 70 of the $76(92 \%)$ with CONC and 11 of the $15(73 \%)$ with DISC who have been recatheterised, fulfilled the criteria for a definitive repair by a modified Fontan operation.

The majority of patients with tricuspid atresia and ventriculoarterial concordance will be suitable for $\overrightarrow{ }$ eventual definitive surgery. Patients with ventriculoarterial discordance, however, often have systemic outflow obstruction at presentation resulting in a much poorer prognosis despite palliative intervention.

Incidence and site of pulmonary stenosis after anatomical correction of transposition of the great arteries

R P Martin, E J Ladusans, J M Parsons, E Keck, R Radley-Smith, M H Yacoub

Harefield Hospital, Middlesex

Supravalvar pulmonary stenosis (PS) is a well recognised complication of anatomical correction of transposition of the great arteries. To investigate the incidence and site of obstruction, we performed right heart catheterisation and right ventriculography in 66 patients between 1.3 and 50 (mean 14) months 9 postoperatively. Pulmonary artery reconstruction had been performed by direct anastamosis as described by Le Compte in 45 patients $(68 \%)$ by direct anastamosis without Le Compte's manoeuvre in seven patients $(11 \%)$; using a dura tube in 11 patients $(17 \%)$, a Dacron tube to the right of the aorta in two patients $(3 \%)$, and with a pericardial tube in one patient $(2 \%)$. The right ventricular sys- 0 tolic pressure for the whole group ranged from 18 to $\mathbb{D}$ 95 (mean 46) $\mathrm{mm} \mathrm{Hg}$. The right to left ventricular systolic pressure ratio (RV:LV) ranged from 0.2 to 0 1.5 (mean 0.5). Significant PS (defined as RV:LV $>0.5)$ was present in 23 patients $(35 \%)$ and was most common in those that had undergone primary cor- $\stackrel{\mathbb{Q}}{\perp}$ rection during the neonatal period (nine of $22 \%$ patients $(41 \%)$ ) compared with those having a two stage operation (eight of 30 patients $(27 \%)$ ). The site 
of obstruction was the pulmonary anastomosis in 14 patients, branch pulmonary arteries in two, dysplastic pulmonary valve in three, and kinking of a Dacron tube in two. In two patients no obvious site of obstruction was identified. Apparent branch pulmonary artery narrowing was present in $59 \%$ of those corrected as neonates compared with $9 \%$ of the remaining patients. The mean pulmonary valve annulus diameter for all patients was $65 \%$ of normal values $(p<0.05)$. Three patients have undergone successful reoperation to relieve severe PS.

Supravalvar PS continues to be an important problem after anatomical correction and further investigation of its aetiology is required.

Can colour flow mapping improve the assessment of lung blood supply in pulmonary atresia with ventricular septal defect?

\section{G R Sutherland, B R Keeton}

Wessex Cardiothoracic Unit, Southampton General Hospital, Southampton

The decision to perform an aortopulmonary shunt in a neonate with pulmonary atresia and ventricular septal defect must be based on accurate knowledge of lung blood supply. Angiography will normally identify adequately sized confluent pulmonary arteries and exclude multiple collateral vessels. Potentially ultrasound imaging with colour flow mapping (CFM) could provide information essential to surgical decision making. Ultrasound information derived from 27 infants has been compared with angiographic data. Imaging consistently predicted the absence of adequately sized central pulmonary arteries in eight patients, identifying the presence of two or more descending aortic collaterals in all such cases. CFM confirmed collateral flow patterns and accurately predicted proximal collateral stenosis. Where central pulmonary arteries were present (and collaterals absent) pulmonary artery continuity was correctly predicted in 14 of 16 patients using imaging alone. CFM confirmed pulmonary artery continuity in all 16. In three patients right and left pulmonary arteries were discontinuous. This was not diagnosed by imaging in any case, but was predicted in all three by CFM. In two additional cases a CFM flow disturbance correctly predicted appreciable central pulmonary artery stenosis.

Major problem areas were defined: (a) imaging did not differentiate dual duct supply from multiple collaterals; (b) imaging could not consistently identify lower thoracic collaterals or those from the head and arm vessels; and (c) the distal morphology of the pulmonary vessels was not defined. In every case, however, sufficient information was derived from imaging plus CFM to allow an accurate non-invasive assessment of the need for aortopulmonary shunting.

\section{Myocardial fibrosis in tetralogy of Fallot: effect of surgery or part of the natural history?}

Anita Hegerty, R H Anderson, J E Deanfield Hospitals for Sick Children, Great Ormond Street, London

Arrhythmia and ventricular dysfunction are recognised complications after repair of tetralogy of Fallot and have been linked to sudden death. We have studied right and left ventricular myocardium in 28 patients with tetralogy of Fallot who died before or at repair ( $<1$ year-11 patients, 1-5 years-12 patients, $>5$ years-five patients) to determine whether myocardial damage occurs as part of the natural history over and above the effects of surgery itself. Semi-automated analysis of fibrous tissue content was performed on sections from right ventricle and left ventricle inlet, apex and outflow tract (60 fields/block), and the results compared with 62 normal hearts. Fibrous tissue in the left ventricle remained normal in all age groups. In contrast, fibrous tissue in the right ventricle was normal in patients $<1$ year $(2 \cdot 1 \mathrm{~g}(0.96)$ in controls and $2 \cdot 2 \mathrm{~g}(1.5)$ in tetralogy of Fallot) but there was an abnormal increase in fibrous tissue with age $(19.8 \mathrm{~g}(9)$ in controls $>5$ years, and $43.3(21)$ in tetralogy of Fallot $>5$ years, $p<0.05$ ). This was maximal in the outflow tract and in the subendocardium.

Progressive damage to the right ventricle occurs with increasing age even before repair of tetralogy of Fallot. This may be the substrate for late postoperative complications and argues in favour of early repair.

\section{Calcific aortic stenosis: a complication of chronic uraemia}

E R Maher, Sara Pugh, G Young,

B Smythe-Walshe, J R Curtis

Departments of Medicine and Cardiology, Charing Cross Hospital, London

The incidence of aortic stenosis (AS) was investigated in a retrospective clinicopathological study of 174 patients with end stage renal disease 
(ESRD). Six patients developed severe calcific AS a mean $9 \cdot 7$ years after starting haemodialysis (HD), which was significantly greater than the expected incidence $(p=0.004)$. In five patients (mean age 50.8 years) AS was caused by severe premature calcification of a tricuspid aortic valve. The aetiology of premature aortic valve calcification (AVC) and stenosis was studied in a prospective echocardiographic study of 87 patients aged between 35 and 70 years on maintenance HD. Five patients had stenosis of a tricuspid aortic valve from premature AVC (mean age 52 years, mean duration of HD 9.4 years). Patients with AVC were older ( 55 and 50 years, respectively, $p<0.02$ ) and had received HD for longer ( 9.4 and 6.8 years, respectively, $p<0.05)$, and had a higher calcium phosphate product $(p<0.001)$ than those without AVC. There was a significant association between the presence of AVC and calcification of the mitral annulus $(p<0.05)$.

Premature AVC is common in patients on long term HD and appears to be associated with abnormal calcium and phosphate metabolism and possibly increased stress on the valve cusps from hypertension and increased cardiac output. In a small but important group of patients severe AVC produces AS. Thus calcific AS can be considered as a complication of ESRD.

\section{Increase in aortic valve gradients with time: a long term retrospective study}

\section{S W Davies, A H Gershlick, R Balcon \\ London Chest Hospital, London}

In patients with valvar aortic stenosis the decision to undertake valve replacement is influenced by the catheter withdrawal gradient. It is unclear whether the gradient increases in patients whose initial gradient indicates that surgery is unnecessary. Records of patients with an aortic valve gradient, who had undergone previous catheterisation, were identified and their initial investigation was reviewed. Thirty four such patients were found; their mean age was 53 years (range 22 to 68 ). The mean initial gradient was $17 \mathrm{~mm} \mathrm{Hg}$ (range 0 to $60 \mathrm{~mm} \mathrm{Hg}$ ) and the mean second gradient was $53 \mathrm{~mm} \mathrm{Hg}$ (range 15 to 120 ). In all but two cases the gradient had increased, and the average rate of change was $+8.0 \mathrm{~mm} \mathrm{Hg}$ per year. Twenty three of the 34 subsequently underwent aortic valve replacement. In 10 of the patients no initial gradient had been found and in this group the mean gradient at reinvestigation was $44 \mathrm{~mm} \mathrm{Hg}$ (range 25 to $65 \mathrm{~mm} \mathrm{Hg}$ ) after 7 years (range two to 10 ). In the 24 patients with an initial gradient, this changed from a mean of $24 \mathrm{~mm} \mathrm{Hg}$ (range 10 to $66 \mathrm{~mm} \mathrm{Hg}$ ) to $56 \mathrm{~mm} \mathrm{Hg}$ (range 15 to $120 \mathrm{~mm} \mathrm{Hg}$ ) after six years (range one to 17). The rate of progression was not significantly correlated with age, the initial gradient, the presence of calcification, or the diagnosis of rheumatic or non-rheumatic heart disease.

Aortic valve gradients which are not important may become so with time. Our results suggest that aortic valve replacement should be considered in patients with minor gradients in whom cardiac surgery is to be undertaken for other reasons.

\section{Doppler echocardiography in aortic valve disease: limitations in the presence of aortic incompetence}

G Karatasakis, P Nihoyannopoulos, Shaughan Dickie, Celia M Oakley

Royal Postgraduate Medical School, Hammersmith Hospital, London

Aortic incompetence (AI) complicates accurate Doppler measurement of systolic aortic gradients. To assess the relation between the severity of $A I$ and errors in the Doppler measurement of peak aortic gradient, we performed continuous wave Doppler one to three days before aortic angiography and haemodynamic measurement of peak to peak aortic valve gradient in 50 patients aged 65 years (range 10-86 years) with aortic valve disease. Doppler acceleration rate was calculated $\left(\mathrm{m} / \mathrm{sec}^{2}\right)$ as the peak velocity $(\mathrm{m} / \mathrm{sec})$ normalised to acceleration time $(\mathrm{sec})$ which is defined as the time from the onset to the peak of systolic aortic flow. The systolic aortic jet was located by colour coded flow mapping. AI was angiographically mild (grade $0-2 / 4$ ) in 35 of 50 patients $(70 \%)$ and moderate to severe in 15 of 50 patients $(30 \%)$. Peak to peak catheter gradient was 54 (24) $\mathrm{mm} \mathrm{Hg}$ (range 10-110) with Doppler calculated peak gradient $74(25) \mathrm{mm} \mathrm{Hg}$ (range 29-118). The Doppler gradient overestimated the catheter gradient by 19 (14) $\mathrm{mm} \mathrm{Hg}$ (range 1-60). Doppler acceleration rate was $40(6) \mathrm{m} / \mathrm{sec}^{2}$ (range $24-55$ ). This was related to the difference in Doppler and catheter gradients $(r=0.55, p<0.001)$ and independent of the severity of aortic stenosis $r=0.07$, (NS). In patients with severe AI the Doppler overestimation was greater $(33(16)$ and $14(8) \mathrm{mm} \mathrm{Hg}$, respectively) ( $\mathrm{p}=0.001)$ and the Doppler acceleration rate was greater $\left(47(3)\right.$ and $37(5) \mathrm{m} / \mathrm{sec}^{2}$, respectively) $(\mathrm{p}<0.0001)$. All patients with severe AI had acceleration rates of more than $45 \mathrm{~m} / \mathrm{sec}^{2}$.

Moderate to severe AI complicates accurate Doppler measurement of systolic aortic gradients. Doppler acceleration rate is a non-invasive index able to depict the contribution of AI to the generation of Doppler gradients. 
Echocardiographic left ventricular wall dimensions to grade the severity of aortic stenosis: a historical myth

\author{
M J Griffith, Cathey Carey, D J Coltart, \\ B S Jenkins, $M$ W Webb-Peploe \\ St Thomas's Hospital, London
}

Left ventricular hypertrophy as characterised echocardiographically is a useful indicator of the presence of aortic stenosis (AS) but is also used to grade the severity of obstruction. To test the validity of this we compared $M$ mode echocardiographic measurements of the septum and posterior wall with Gorlin aortic valve area (GAVA) derived at cardiac catheterisation. Three hundred and thirty patients with good quality echocardiograms who underwent cardiac catheterisation for AS were studied. Patients with grade 3 or 4 aortic regurgitation or no significant aortic gradient were excluded. The GAVA was calculated from the mean aortic valve gradient, systolic ejection period, and dye dilution derived cardiac output. The correlation was then calculated between the GAVA and the left ventricular wall dimensions. The correlation coefficients were poor, $r=0.13$ for the septum and $r=0.15$ for the posterior wall. The maximum diagnostic accuracy for severe AS (defined as a GAVA $<0.70 \mathrm{~cm}^{2}$ ) for the range of wall dimensions was $16 \%$ for a septum $>1.2 \mathrm{~cm}$ and $15 \%$ for a posterior wall $>1 \cdot 2 \mathrm{~cm}$. The correlation with other haemodynamic variables was better, peak left ventricular systolic pressure having $r$ values of 0.37 and 0.30 for posterior wall and septum, respectively. Mean and peak aortic valve gradient had $r$ values approaching 0.30 for both dimensions. The correlation coefficient with systolic arterial pressure and cardiac index approached zero.

These data demonstrate that the left ventricular wall dimensions correlate poorly with GAVA and should not be used to grade AS. As the wall dimensions correlate better with the aortic valve gradients than the valve area they will tend to compound any error in grading aortic stenosis when the area is not calculated.

\section{Tolerance to nitrates: does it occur and how long a nitrate free period is required to prevent it?}

M A James, M Papouchado, J V Jones

Cardiology Department, Bristol Royal Infirmary, Bristol

We have investigated whether the loss of therapeutic effect of transdermal nitrate preparations is due to tolerance (rather than system failure), how rapidly this tolerance develops, and how long a nitrate free period is required to prevent it.

A randomised, double blind, crossover trial studied exercise tolerance during treatment with $10 \mathrm{mg}$ glyceryl trinitrate patches for two consecutive days on four successive weeks in 12 patients with angina (mean age 57.5 years). Three active or dummy patches were applied continuously for 32 or 36 hours. Exercise testing was performed at 1200 and 1700 or 2100 hours on each day. The timing and order of active and dummy patches varied with each treatment period so that the effect of continuous treatment was tested at $3,8,12,15$, and 24 hours, and intermittent treatment on two consecutive days using two different nitrate free periods ( 12 hours or 16 hours). The results showed a significant treatment effect at three and eight hours, a reduced effect at 12 and 15 hours which was no longer significant and by 24 hours there was no effect at all. Thus during continuous treatment tests on day two were significantly worse than on day one and no better than placebo. Both nitrate free periods prevented the development of tolerance such that tests on day two remained significantly better than placebo. Although exercise measurements on day two were slightly worse than on day one during intermittent treatment this was not significant overall.

This study confirms that loss of effect from transdermal nitrates is due to tolerance, that its onset is between 8-12 hours but is not complete until after 15 hours. It is prevented by nitrate free periods, and 12 hours may be sufficient although there was a suggestion that this was less effective than 16 hours.

Efficacy of captopril and enoximone in stable severe heart failure

\author{
R J I Bain, N H Anfilogoff, M K Davies, \\ R G Murray, W A Littler \\ Department of Cardiovascular Medicine, East \\ Birmingham Hospital, Birmingham
}

The relative efficacy of captopril $25 \mathrm{mg}$ three times a day (C), an established ACE inhibitor, and enoximone, a new inotrope, $100 \mathrm{mg}$ three times a day (E), was tested in 12 patients with stable New York Heart Association (NYHA) classes III-IV heart failure. A randomised, double blind, crossover protocol was adopted and haemodynamic data recorded at rest and exercise before randomisation and at the end of each six week treatment phase. Cardiac power output derived from pre load arterial pressure and cardiac output was used as a measure of cardiac per- 
formance. Although NYHA class improved on both drugs from a mean (SE) of $3.4(0.1)$ to $2.7(0.2)(\mathrm{C})$ and $2.5(0.2)(E), p<0.02$, grouped data failed to show improvement in exercise time, or rest or exercise haemodynamics. Exercise time, however, improved by $>20 \%$ in six patients; five on enoximone and one on captopril. At baseline these responders $(R)$ to treatment had a greater capacity to increase cardiac output with exercise $(\triangle \mathrm{CPO}=\mathbf{0 . 8 8}$ watts $(\mathrm{R}))$ compared to non-responders (NR) $(0.55$ watts). All five who responded to the inotrope did so following six weeks of captopril treatment and their increase in exercise capacity was matched by an enhanced exercise related increase in CPO $(\Delta \mathrm{CPO}=1.15(\mathrm{R})$ vs. 0.64 (NR) watts).

These results suggest that enoximone may benefit some patients by increasing their functional cardiac reserve. The responders received captopril before the inotrope suggesting that prior ACE inhibition may enable the heart to respond to inotropic stimulation, perhaps mediated through up regulation of the $\beta$ receptor.

\section{Isoprenaline, dopamine, and dobutamine infusions all cause hypokalaemia}

M Smith, D R Corfield, Helen Drake, T Treasure Departments of Medicine and Cardiothoracic Surgery, The Middlesex Hospital, London

Swings in potassium accompany infusions of adrenaline and isoprenaline but the commonly used inotropes, dopamine and dobutamine, were thought not to cause hypokalaemia. We have tested the effect of these drugs on short term potassium homeostasis in a controlled manner in dogs. Isoprenaline $(0 \cdot 1 \mu \mathrm{g} / \mathrm{kg} / \mathrm{minute})$, dopamine, and dobutamine (both $15 \mu \mathrm{g} / \mathrm{kg} /$ minute) were given by infusion over 30 minutes while arterial potassium changes were followed continuously with a catheter tip potassium selective electrode. Infusions were preceded and followed by 30 minute control periods of $0.9 \%$ saline infusion. Eight infusions of each drug were given to six adult beagles. As with other catecholamines, there was a consistent, transient, but small rise $(0 \cdot 1 \mathrm{mM})$ in potassium concentration with all three drugs followed by a more profound hypokalaemia which was sustained for the duration of the infusion The mean (1 SD) fall in potassium concentration was $0.80(0.19) \mathrm{mM}$ for isoprenaline, $0.71(0.26) \mathrm{mM}$ for dopamine, and $0.68(0.29) \mathrm{mM}$ for dobutamine, and over $1.0 \mathrm{mM}$ in some instances with all three drugs. Ventricular ectopy developed in two dogs during dopamine infusion and resolved during the control (saline) infusion when the potassium concentration was returning towards normal.

The potential implications of these findings on clinical practice have encouraged us to investigate the possibility that similar changes occur in man.

\section{Effect of low maintenance dose $\Omega 3$ fatty acids on serum lipid concentrations}

\author{
Suad Ismail, Marie Brannigan, D O'Callaghan, \\ J H Horgan \\ Department of Cardiology, St Laurence's Hospital, \\ Dublin, Ireland
}

A fish oil concentrate rich in eicosapentaenoic and docosahexaenoic acids has been shown to reduce serum cholesterol and triglycerides and raise high density lipoprotein concentrations. These studies have entailed ingestion of large quantities of fish oil which has proved unacceptable to many patients. This pilot study was designed to assess the effects of lower doses of fish oil. We studied 21 patients who had raised cholesterol and triglyceride concentrations despite adherence to an individualised restricted diet. The patients were divided into two comparable groups, $A$ and $B$, and the study was performed in a modified double blind crossover fashion. Group A (10 patients) received a loading dose of $3000 \mathrm{mg}$ ( 10 capsules) of fish oil daily (a combination of $180 \mathrm{mg}$ of eicosapentaenoic acid and $120 \mathrm{mg}$ of docosahexaenoic acid per capsule) for one week, followed by $900 \mathrm{mg}$ (three capsules) daily for 12 weeks. They then received three placebo capsules daily for a further 12 week period. Group B (11 patients) received 10 placebo capsules daily for a week, followed by three placebo capsules daily for 12 weeks, continuing to $900 \mathrm{mg}$ fish oil (three capsules) daily for 12 weeks. Results showed a significant reduction in serum triglyceride concentrations in group A over the initial loading week $(p<0.05)$. This reduction was maintained by the lower dose over the following 12 weeks ( $p<0.01$ over the 13 week period). There was no significant change in serum triglyceride concentrations in group B (no loading period) or in the placebo treated group. There was a significant rise in high density lipoprotein concentrations in group A over the loading week only $(p<0.02)$ but this rise was not maintained over the following 12 weeks ( $p=$ NS over the 12 week period). No change in high density lipoprotein concentrations in group B were observed. No significant changes in serum cholesterol or low density lipoproteins were noted in either group. 
These results suggest that short term high dose fish oil with lower maintenance doses is effective in reducing hypertriglyceridaemia. No effect on total cholesterol was seen. It would appear that high maintenance doses of fish oil are required to sustain a rise in high density lipoprotein concentrations.

\section{Calcium exchange in rabbit myocardium during and after hypoxia: role of sodium calcium exchange}

\section{T Crake, P A Poole-Wilson}

The Cardiothoracic Institute and National Heart Hospital, London

The association between calcium uptake on reperfusion or reoxygenation of myocardium and the recovery of contraction is disputed and the cause unknown. Sodium calcium exchange is a potential mechanism. Calcium uptake was measured using ${ }^{47} \mathrm{Ca}^{2+}$ in the isolated and arterially perfused interventricular septum of the rabbit. Temperature was $35^{\circ} \mathrm{C}$ and the stimulation rate was 90 beats/minute. No change in tissue uptake of calcium occurred during substrate free hypoxia $(n=7)$ for 45 minutes but on reoxygenation tissue calcium uptake increased by $73(7) \%$. Recovery of mechanical function was inversely related to the increase in tissue calcium. Stimulation of sodium calcium exchange by perfusion with a sucrose solution low in sodium, and without potassium, $(n=10)$ increased tissue calcium by between 18 and $136 \%$ and the relation to mechanical recovery was similar to that observed on reoxygenation after a period of hypoxia. Sodium calcium exchange could be stimulated during hypoxia $(n=6)$. Lithium $(100 \mathrm{mmol} / \mathrm{l} / \mathrm{second})$ substitution for extracellular sodium inhibited sodium calcium exchange under control conditions $(\mathbf{n}=3)$. Substituting lithium ( $100 \mathrm{mmol} / 1 / \mathrm{second})$ for sodium $(100 \mathrm{mmol} / \mathrm{l} / \mathrm{second})$ during the last 35 minutes $(n=6)$ and the last 10 minutes $(n=8)$ of the 45 minute period of hypoxia and the first 20 minutes of reoxygenation resulted in an increase in ${ }^{47} \mathrm{Ca}^{2+}$ uptake of $69(9) \%$ and $79(9) \%$, respectively, similar to when lithium was not used. Recovery of mechanical function was not affected by lithium. Inhibition of sodium hydrogen exchange during hypoxia and reoxygenation by amiloride $\left(10^{-4} \mathrm{mmol} / 1 /\right.$ second $)$ had no effect on the reoxygenation induced uptake of calcium.

The increase in calcium uptake that occurs on reoxygenation after a period of substrate free hypoxia in this experimental model is related to the recovery of mechanical function, but sodium calcium exchange is not a major mechanism.
Accelerated intraventricular systolic flow differentiates patients with hypertrophic cardiomyopathy from those with secondary causes of hypertrophy

P Nihoyannopoulos, Y Yonezawa, Shaughan Dickie, W J McKenna, Celia M Oakley

Royal Postgraduate Medical School, Hammersmith Hospital, London

The diagnosis of hypertrophic cardiomyopathy (HCM) is based on being able to show unexplained left ventricular hypertrophy (LVH). Echocardiography is the best technique for detecting LVH but it is not specific for the diagnosis of HCM as opposed to other causes of hypertrophy. To determine whether the intraventricular flow profile is specific in HCM we performed intraventricular flow mapping in 27 patients with HCM (14 with and 13 without left ventricular (LV) gradient), 25 patients with secondary LVH (10-aortic stenosis, 10-systemic hypertension, five-cardiac amyloidosis) and 10 patients with mitral regurgitation. Ten normal subjects were studied for comparison. A Toshiba SSH 65. A was used at a fixed PRF (4 KHz) with a $2.5 \mathrm{MHz}$ transducer. The presence and position of intraventricular aliasing or turbulent (mosaic) flow on colour Doppler was noted. Then sequential peak velocities $(\mathrm{cm} /$ second) were obtained with unigate pulse Doppler at the apex, papillary muscles, LV outflow tract, and ascending aorta. Patients with HCM (apex 36 (5), papillary muscle 78 (16), LV outflow 212 (154), aorta 104 (13) had significantly higher intraventricular flows when compared with aortic stenosis (26 (4), 41 (5), 67 (4), and 390 (42), $\mathrm{p}<0.001$ ), hypertensives (28 (3), $48(6), 72$ (7), and $95(6), \mathrm{p}<0.001)$, cardiac amyloid (22 (2), 34 (3), 63 (3), and $72(5), p<0.00001)$, mitral regurgitation (29 (4), 54 (15), 75 (4), and $96(6), p<0.001)$ and the normal individuals (23 (2), 41 (5), 67 (9), and 96 (1), $\mathrm{p}<0.001)$. There was no overlap of peak intracavitary flow velocities in HCM when compared with secondary LVH. Thirteen patients, however, with HCM and no gradient fell within the ranges detected in mitral regurgitation.

Patients with HCM have increased velocities with progressive acceleration of intraventricular flow which distinguish them from secondary LVH.

\section{Magnetic resonance imaging of infants with congenital heart defects}

E J Baker, M A Smith, R H Anderson, M J Tynan, Victoria Ayton

Guy's Hospital, London 
We have studied 12 infants with various congenital heart defects (aged 4 days to 3 months) by cardiac magnetic resonance imaging (MRI). A 1.5 Tesla whole body imaging system and a $32 \mathrm{~cm}$ diameter head coil were used. Eight were studied without sedation. Five millimetre electrocardiogram gated sections were acquired using a spin echo sequence. A matrix size of $256 \times 256$ was used. Oblique imaging was used to provide optimal imaging of cardiac malformations. Typical scan time for a series of seven sections was six minutes. The images were of good quality in all cases. MRI was found to be of special value in studying seven patients with suspected abnormalities of the aortic arch. The anatomy of proximal pulmonary arteries was well shown including a Blalock Taussig shunt in one patient. Absence of central pulmonary arteries and the presence of collateral arteries was shown in a further case. In two patients with ventricular septal defects the anatomy was well defined, in one case showing multiple trabecular defects which had not been evident on angiography. Systemic and pulmonary venous connections were well shown, including one case of anomalous pulmonary venous connection. The morphology of atrial and ventricular chambers was clearly delineated. The demonstration of the arterial connections was excellent in a case of double outlet of the right ventricle. Resolution was not adequate to identify an anomalous coronary artery in one case.

Superior resolution with high field strength MRI systems make them especially suitable for the investigation of heart defects in this age group.

\section{Atrial natriuretic peptide in spontaneous tachycardia}

I G Crozier, M G Nicholls, H Ikram, E A Espiner St George's Hospital, London, and the Princess Margaret Hospital, Christchurch, New Zealand

Venous atrial natriuretic peptide concentrations (ANP) were measured by radioimmunoassay during and following conversion of spontaneous tachycardias, to determine the effect of tachycardia on ANP. We studied 34 consecutive patients, aged 29-84 years, with spontaneous tachycardias of 0.5 hours to 100 days duration. Sinus rhythm was restored by direct current cardioversion under thiopentone anaesthesia $(n=18)$, pharmacologically $(n=13)$, or spontaneously $(n=3)$. ANP during tachycardia ranged from 17-525, mean (SEM) (140 (23) $\mathrm{pmol} / \mathrm{l}$, and was highest in ventricular tachycardia (332 (58) pmol/l, $\mathrm{n}=7$ ), and concentrations in supraventricular tachycardia (120 (39) pmol/l, $\mathrm{n}=5$ ) and atrial fibrillation (85 (11) pmol $/ 1, \mathrm{n}=22$ ) were higher than normal (range 8-24 pmol/1). ANP was higher in patients with structural cardiac disease than in those without $(170$ (28) and 59 (21) pmol/l, respectively, $p<0.05)$. ANP and systolic arterial pressure during tachycardia correlated negatively $(\mathrm{r}=-0.60, \mathrm{p}<0.001)$; ANP, however, was only weakly related to the ventricular rate $(r=0.31, N S)$ and the duration of the tachycardia $(r=-0 \cdot 18, N S)$. After conversion of the tachycardia to sinus rhythm ANP fell to 116 (17) pmol/1 at five minutes and 101 (19) $\mathrm{pmol} / 1$ at 15 minutes $(\mathrm{p}<0.01$ and $\mathrm{p}<0.02$, respectively, compared with ANP during tachycardia).

We conclude that ANP is raised during tachycardia with the highest concentrations being observed during ventricular tachycardia and in patients with structural cardiac disease. The ANP concentrations observed during tachycardia are within a range known to have biological effects in man.

\section{Are changes in plasma catecholamine concentrations responsible for the failure of acute phase $\beta$ blockade to limit infarct size?}

D P Murray, R D S Watson, R G Murray, W A Littler

University Departments of Cardiovascular Medicine, East Birmingham and Dudley Road Hospitals, Birmingham, and Cork Regional Hospital, Cork, Ireland

Despite a $15 \%$ reduction in mortality, neither the MIAMI nor ISIS-1 trials reported a significant reduction in enzymatically estimated infarct size in patients receiving $\beta$ blockers. Therefore 20 patients with acute myocardial infarction were randomised blindly to treatment with intravenous, followed by oral, metoprolol or placebo. Patients were haemodynamically monitored for $\mathbf{2 4}$ hours. Plasma adrenaline $(\mathrm{AD})$ and noradrenaline $(\mathrm{NAD})$ concentrations were estimated at baseline (mean $6.0(0.9)$ hours from onset of symptoms), and one and 24 hours from start of treatment. Plasma AD and NAD were raised in all but one patient, with a further increase in those receiving metoprolol $(p<0.05)$. At baseline pulmonary wedge pressure was directly related to both $\mathrm{AD}(\mathrm{r}-0.44 ; \mathrm{p}<0.05)$ and NAD concentrations $(\mathrm{r}-0.44 ; \mathrm{p}<0.05)$ while stroke volume index was inversely related to NAD $(r-0.44 ; p<0.05)$. From baseline to one hour the change in systemic vascular 
resistance was related to the increase in both $\mathrm{AD}$ $(\mathrm{r}-0.48 ; \mathrm{p}<0.05)$ and NAD concentrations $(r-0.66 ; p<0.01)$.

In acute myocardial infarction high plasma catecholamine concentrations were associated with the haemodynamic markers of severe left ventricular damage. $\beta$ blockade produced a further rise in catecholamine concentrations which was associated with a rise in systemic vascular resistance. The resulting increase in myocardial oxygen consumption may, in part, explain the limited impact of $\beta$ blockade on myocardial salvage in acute infarction.

\section{Myocardial dysfunction in ankylosing spondylitis}

\section{B A Gould, J Turner, D H Keeling, P Hickling, A J Marshall \\ Departments of Cardiology, Rheumatology, and Nuclear Medicine, Plymouth General Hospital, Plymouth}

Echocardiographic evidence has suggested there are abnormalities of myocardial function in patients with ankylosing spondylitis (AS). We have further evaluated cardiac function in patients with $A S$ and in normal volunteers (NV). We studied $24 \mathrm{NVs}$ and 21 patients with AS in the age range 18-45. None had overt cardiac disease. Cardiac function was assessed at rest and during supine bicycle exercise using radionuclide angiography in the LAO position following equilibration with $740 \mathrm{MBq}$ of technetium-99. Subjects undertook supine bicycle exercise with 30 watt increments every three minutes to the point of fatigue. Comparisons of NV and AS patients' data were made using the Student's $t$ test for independent samples or the Mann-Whitney nonparametric test, as appropriate. Subjects were matched for age, sex, height, and weight. Global left ventricular function showed several differences between NV and AS. Peak filling rate during exercise was significantly lower in AS (NV 6.5, (SD 1.2); AS $5 \cdot 7(1 \cdot 2), p=0 \cdot 026)$. Time to reach peak filling during exercise was significantly lower on exercise in AS (NV 102, (22), AS 120 (23), p = 0.012). Wall motion was abnormal at rest and on exercise in AS $\mathbf{p}=0.00004$. Regional analysis also showed differences between AS and NVs both at rest and on exercise. In the anteroseptal region, filling fraction and peak filling rate were significantly lower in AS $p=0.044$. Most of the differences were in variables of diastolic function.

This study shows that there are abnormalities in cardiac function in AS. The major abnormalities are in diastolic function suggesting a decrease in $\mathrm{LV}$ compliance.

\section{Hypercoagulability, consumptive coagulopathy, and paradoxical reduction of platelet aggregation in infective endocarditis}

T Taha, S Durrant, J Crick, Celia M Oakley Departments of Clinical Cardiology and Haematology, Royal Postgraduate Medical School, Hammersmith Hospital, London

Local and generalised changes in coagulation may be important in the genesis of vegetations and embolism in infective endocarditis (IE). To characterise such alterations, serial haematological investigations were performed on consecutive cases of IE. Preliminary data are presented here as clear patterns of abnormality emerged quickly. Platelet survival was measured by indium-111 labelling. Acute and convalescent samples were analysed for fibrinogen, factor VIII, antithrombin III (AT3) and fibrin degradation products (FDPs) by standard methods, as well as adenosine diphosphate and collagen induced platelet aggregation. Five patients had a high fibrinogen concentration (5.0-10.6, mean $8.02 \mathrm{~g} / \mathrm{dl}$ normal range, $\overline{\mathrm{n}}, 2-4)$ and a high normal factor VIII concentration $(120-180 \%$, mean $144 \% \overline{\mathbf{n}}$ $50-200 \%$ ) with a drop in each case in convalescent samples to $(3 \cdot 5-4 \cdot 7$, mean $4 \cdot 12 \mathrm{~g} / \mathrm{dl})$, $(90-120 \%$, mean $104 \%$ ), respectively. Their AT3 were normal. FDPs were at the upper limit of normal $(8 \mu \mathrm{g} / \mathrm{ml}$ $\overline{\mathbf{n}}<8$ ) in one early sample but otherwise normal. In two patients fibrinogen concentration was lower $(2 \cdot 4,4 \cdot 8 \mathrm{~g} / \mathrm{dl})$ acutely as was factor VIII $(83,91 \%)$, with a later rise to $(4 \cdot 5,7 \cdot 6 \mathrm{~g} / \mathrm{dl})$ and $(108-140 \%)$, respectively. Their AT3 was low $(63,75 \%)$ and FDPs high $(64,16 \mu \mathrm{g} / \mathrm{ml})$ acutely, returning to normal in the convalescent sample. In all patients platelet half life was moderately reduced (3.4-4.9 days, $\overline{\mathrm{n}}$ 5-6 days). Platelet aggregation was reduced acutely with ADP in six patients and collagen in five, but normal subsequently.

The results suggest that in the active stage of IE: (a) hypercoagulability may be caused by a rise in acute phase reactants, (b) subclinical consumptive coagulopathy may supervene and reverse this tendency, and (c) there is an unexpected reduction in platelet aggregation possibly as a result of continued circulation of previously activated "exhausted" platelets. 


\section{Enzymatic differentiation of cardiac and skeletal muscle}

Jane Flint, D A Robertson, J M Parmar, Karen Falholt, Lisa Heding, M Nattrass, B L Pentecost

General and Queen Elizabeth Hospitals, Birmingham, and the Novo Research Institute, Copenhagen

Cardiac muscle differs from other striated muscle in preferred substrates. In normal myocardium energy is obtained primarily from lipid metabolism. To assess any differences in intracellular enzyme activities, 26 patients undergoing thoracotomy (13 for coronary artery bypass grafting, and 13 for aortic valve replacement) had right atrial appendage and intercostal muscle biopsy specimens taken before going on to bypass. Lipid and enzyme content was determined after a Folch extraction. Known diabetic and hyperlipidaemic patients were excluded. Lipid content was significantly greater in atrial muscle: triglyceride concentrations expressed as mean (SEM) were 308 (67) and 69 (14) $\mu \mathrm{mol} / \mathrm{g}$, respectively, $\mathrm{p}<0.01$; non-esterified fatty acid concentrations were $0.84(0.10)$ and $0.35(0.05) \mu \mathrm{mol} / \mathrm{g}$, respectively, $p<0.001)$. In tissue homogenates enzyme activities ( $U / g$ wet weight) differed significantly. Glycolytic enzyme activity was lower in atrial muscle: hexokinase $1.19(0.25)$ and 1.86 $(0.15)$, phospho-fructokinase $0.44(0.03)$ and 2.78 $(0.03)$, pyruvate kinase $7.8(0.4)$ and $14.3(1.0)$, and glycerol 3 phosphate dehydrogenase $3.8(0 \cdot 2)$ and $15 \cdot 2(1 \cdot 1)$, (all p $<0.001$ ). Enzymes of fat metabolism had significantly greater activity in atrial muscle: glucose 6 phosphate dehydrogenase $0.43(0.03)$ and $0.20(0.02)$, malic enzyme $0.16(0.01)$ and 0.08 $(0.01), 3$ hydroxybutyrate $\mathrm{CoA}$ dehydrogenase 8.6 $(0.5)$ and $3.8(0.3)$, (all $p<0.001$ ).

These results support the increased importance of lipid metabolism and reduced role for glycolysis in cardiac muscle compared with other striated muscle.

Financial and resource considerations for initiating a coronary angioplasty service

M F Shiu, Faith Bailey

University Department of Cardiovascular Medicine, Queen Elizabeth Hospital, Birmingham

To date most cardiac centres starting coronary angioplasty have accommodated it within existing departmental facilities and resources. The cost and staffing requirements of such a programme are examined in this review of our four years experience in providing a regional coronary angioplasty service. From 1983 to 1986 a total of 243 procedures was performed at an estimated cost of $£ 286000$ to the unit. This included actual expenditure in disposable angioplasty equipment $(£ 126000)$, additional nursing and technical staff $(£ 63000)$, and the cost of hospital stay $(£ 97000)$, but exclude the cost of six emergency bypass graft operations. The average cost of $£ 1180$ per procedure probably represents the minimum cost for coronary angioplasty without additional medical staff or capital cost of an interventional $x$ ray laboratory. Our experience is that up to 150-200 angioplasty procedures may be accommodated in this way in a diagnostic cardiac laboratory by means of additional ancillary. staff and rescheduling of diagnostic sessions.

As the indications for the procedure broaden it will almost certainly require purpose built interventional facilities, at which point the capital and staffing costs of the procedure will need further evaluation.

Does early $\beta$ blockade reduce mortality in myocardial infarction by limiting infarct size?

D P Murray, R G Murray, W A Littler University Departments of Cardiovascular Medicine, East Birmingham Hospital, Birmingham, and Cork Regional Hospital, Cork, Ireland

The mechanism by which acute phase $\beta$ blockade reduces mortality in acute myocardial infarction is unclear. Therefore in a double blind placebo controlled study the effects of intravenous, followed by oral, metoprolol on peak serum lactic dehydrogonase (LDH) activity and aspartate aminotransferase (AST) activity, QRS score on ECG and thallium201 (TL) perfusion defect score were studied in 129 patients with a median delay of 6.75 hours from the onset of symptoms of infarction. Peak LDH activity correlated with both QRS $(\mathrm{r}-0.40, \mathrm{p}<0.001)$ and TL score $(r-0.44, p<0.001)$, as did peak AST activity $(r-0.37$ and 0.41$)$. While metoprolol did not affect these correlation coefficients it did influence the slope of regression lines, reducing peak LDH activity by $7 \%$ (90\% confidence limits -475 to $+199 \mathrm{iu})$ and peak AST activity by $11 \%(90 \%$ confidence limits -65 to $+27 \mathrm{iu}$ ). Metoprolol did not reduce QRS score or TL defect size. Thus it appears that the reduction in enzyme release was not due to myocardial salvage.

It seems likely that the reduction in mortality after early intervention with metoprolol in myocardial infarction is due, not to limitation of infarct size, but to some other mechanism. 
Cine magnetic resonance imaging of the cardiovascular system

S R Underwood, D N Firmin, R Mohiaddin, R H Klipstein, H Bogren, R S O Rees, D B Longmore

Magnetic Resonance Unit, National Heart and Chest Hospitals, London

Cine magnetic resonance imaging using a field echo sequence with even echo rephasing has the same acquisition time as conventional static imaging (three minutes) but produces $40 \mathrm{~ms}$ frames representing an average cardiac cycle. It has three principal advantages: the integration of information from many parts of the cardiac cycle increases effective resolution, the high signal from blood aids the detection of intracardiac filling defects, and flow patterns can be assessed because of loss of signal from turbulent flow. We have used cine imaging in 100 patients and have identified four areas in which it is of particular value: (a) assessment of global and regional ventricular function can be made from a single oblique acquisition of approximately three minutes, and area length left ventricular volume measurements correlated well with the accurate multislice technique in 25 normal subjects and 20 with previous infarction $(r=0.96$ SEE $=10 \mathrm{ml})$; (b) filling defects such as thrombi $(n=$ three $)$ and the intimal flap of aortic dissection $(n=$ three) were easily seen because of the high signal from blood and the lower signal from all other cardiac structures; (c) abnormal valve motion and the turbulent flow of valvar disease $(n=30)$ can be detected together with shunting through septal defects and surgical conduits $(n=6)$; and (d) the pericardium can be distinguished from pericardial fluid $(n=4)$, and the distinction between pericardial constriction and cardiac tamponade is simplified.

Cine imaging is an important advance that enhances the information from cardiovascular magnetic resonance studies in a large proportion of cases.

\section{Rapid neointimal and medial hyperplasia after arteriovenous bypass in pigs}

\author{
G D Angelini, H Williams, A C Newby \\ Department of Cardiology, University of Wales \\ College of Medicine, Cardiff, Wales
}

Neointimal hyperplasia restricts flow and predisposes to thrombosis in coronary vein grafts. To investigate its mechanism and how it can be reduced, we established an experimental model in which a $3 \mathrm{~cm}$ long segment of autologous pig saphenous vein was grafted into a carotid artery. The vein was anastomosed end to end using bevelled ends and 7-0 continuous prolene sutures. Freshly isolated vein and grafts removed after one week were perfusion fixed at $100 \mathrm{~mm} \mathrm{Hg}$ with $10 \%$ phosphate buffered formalin. A segment of freshly isolated vein and two to four segments from each graft were embedded in paraffin, stained with Van Gieson's elastic stain, and three transverse sections at each of three or four levels were analysed by computer aided planimetry using the endothelium, internal, and external elastic laminae to delimit the lumen, intima, and media, respectively. Cell viability was determined in extracts of unfixed vein and grafts frozen in liquid nitrogen by measurement of the ATP:ADP ratio. Freshly isolated vein $(n=11)$ had a luminal area of $2 \cdot 1(0.5)($ SEM $) \mathrm{mm}^{2}$, an intimal area of 0 , a medial area of $0.7(0.1) \mathrm{mm}^{2}$, and an ATP:ADP ratio of 3.3:0 1 $(n=19)$. Grafts after one week $(n=7)$ had a luminal area of $10(1) \mathrm{mm}^{2}$, an intimal area of 0.4 $(0.2) \mathrm{m}^{2}$, a medial area of $2.8(0.4) \mathrm{mm}^{2}$, and an ATP:ADP ratio of $2 \cdot 7: 0 \cdot 2 \quad(n=14)$. The data showed significant $(p<0.05)$ increases in luminal, intimal, and medial cross sectional areas in grafted vein with maintenance of cell viability. Preliminary results on three grafts removed after one month showed further mean increases in intimal area to $2.6 \mathrm{~mm}^{2}$ and in medial area to $5.5 \mathrm{~mm}^{2}$.

The model thus reproduces the rapid intimal and medial proliferation seen in human coronary artery bypass grafts.

\section{$T$ wave normalisation on exercise: a non-specific finding after myocardial infarction}

L Hughes, P DasGupta, A Lahiri, E B Raftery Northwick Park Hospital and Clinical Research Centre, Harrow

Exercise induced ST segment shift is a marker for ischaemia in patients with heart disease. Following myocardial infarction, some patients do not exhibit ST segment shift despite developing effort angina, and a proportion of these 'normalise' inverted $T$ waves on exercise. To ascertain the importance of such ECG changes, 11 patients who showed repeatable normalisation of $T$ wave on symptom limited exertion (with no ST segment shift) underwent high resolution stress thallium-201 imaging, with simultaneous ECG recording of leads CM5 and CC5. In five patients the end point was angina; the remain- 
der, fatigue or breathlessness. Seven patients had unequivocal $Q$ wave infarction and the remaining four patients had documented myocardial infarction but without $Q$ wave or ST segment change at the time of assessment. Four patients (all with $Q$ wave infarction) had evidence of infarction on thallium scan with no reversible ischaemia, and none of these developed angina on stress. The remainder all had evidence of infarction and reversible ischaemia of variable severity. Of the remaining three $Q$ wave infarcts, two had angina, and three of the four non $Q$ wave infarcts who showed reversible ischaemia on thallium scanning stopped with angina.

We conclude that normalisation of the $T$ wave as an isolated finding does not imply reversible ischaemia especially in the presence of $Q$ wave infarction and is a non-specific finding after myocardial infarction.

\section{Cardiorespiratory abnormalities to imagined psychological stress: assessment of vulnerability}

Leisa Freeman, A V Conway, P G F Nixon Department of Cardiology, Charing Cross Hospital, London

Situations of imagined psychological stress and exercise were suggested under hypnosis in 30 subjects (19 men; 11 women) using a previously published protocol. Continuous monitoring of three physiological variable were made. Fifteen patients had angiographically documented coronary artery disease (CAD), and 15 documented arrhythmias. Two personality questionnaires were completed. Twenty three $(76.6 \%)$ patients had abnormal physiological responses to the psychological stress presented. Seventeen patients responded with hyperventilation, the mean fall in Petco 2 was 13.9 (6) $\mathrm{mm} \mathrm{Hg}$ compared with $5.1(3.4)(p<0.001)$. Nine of these also had ECG abnormalities: SVT in three, ST elevation in one, ventricular bigemini in two, and $S T / T$ wave abnormalities in three. Frequent extra systoles ( $>10 /$ minute) and bigeminy occurred in a further six patients in response to psychological stress. Seven patients developed hypocapnia at the thought of exercise; all had reproduced chest pain following a previous forced $\mathrm{HV}$ provo-cation test; $\mathrm{CAD}$ was present in six. An apologetic/self-effacing personality was significantly associated with physiological response to psychological stressors (emotion/exercise). This was associated with hostility in the patients with CAD and competitiveness in those with arrhythmias. The discriminant func- tion score from the type A questionnaire predicted responders to psychological stress and exercise in the patients with CAD but not those with arrhythmias.

We suggest that it may be possible to determine groups of patients with heart disease who are particularly vulnerable to psychological stress.

\section{Colour flow imaging of jet direction in aortic and mitral regurgitation: its clinical importance}

P Nihoyannopoulos, Jayshree Joshi, G Karatasakis, Celia M Oakley

Royal Postgraduate Medical School, Hammersmith Hospital, London

Colour flow imaging has provided new insights into spatial distribution of regurgitant flows. We evaluated the direction of aortic (AR) and mitral (MR) regurgitant flows to determine whether colour flow mapping can reveal consistent and specific patterns of spatial flow distribution. From a total of 87 patients, 63 had AR while MR was detected in 53. Twenty five patients had rheumatic heart disease (RHD); all had both AR and MR. AR was directed towards the mitral leaflet tip while the MR was directed posteriorly towards the left atrial back wall in all patients. Eight patients had congenital valve disease; six had bicuspid valve with jet direction along the mitral leaflet, and two had cusp fenestrations with jet directions toward the mitral valve tip. Nine patients had calcific aortic stenosis and mild to moderate AR, with jet directions along the anterior mitral leaflet $(n=15)$, mitral leaflet tip $(n=3)$, and anteriorly along the ventricular septum $(n=1)$. Twelve patients had AR secondary to collagen $(n=2)$, inflammatory disorder $(n=6)$, or dilated cardiomyopathy $(n=4)$. In all, the jet was rising from the cusp tips and was directed towards the mitral leaflet tips, similar to RHD. In six patients with anterior leaflet mitral prolapse the MR jet was directed immediately posteriorly. Conversely, in eight patients with leaflet mitral prolapse the jet was directed anteriorly against the posterior aortic wall. In patients with functional MR secondary to ischaemia $(n=10)$, or dilated cardiomyopathy $(n=4)$, jet direction was posterior, rarely exceeding $30 \%$ of the left atrium.

The flow direction together with the valve morphology is strongly indicative of the aetiology of the underlying disease except in calcific AR when the jet may follow any direction. 
Single vessel coronary disease: what factors influence prognosis?

C Jones, A H Gershlick, Denise Syndercombe-Court, R Balcon The London Chest Hospital, London

Although single vessel disease (SVD) is often considered to have a relatively good prognosis, it is associated with increased mortality. In order to identify those at risk, a retrospective analysis of 884 patients with SVD was undertaken to ascertain which factors might predict death. All patients had had exercise stress tests and angiography. During a mean follow up period of 45 months $48(5.7 \%)$ died, compared with 13 deaths $(1.8 \%)$ in a group of 773 patients without significant coronary disease $(p<0.01)$. Mortality in the SVD group was more common in the 330 patients with impaired left ventricular (LV) contraction (group 1) than among the 554 patients with normal LV function (group 2) $7.6 \%$ and $4.2 \%$, $\mathrm{p}<0.05)$. Within group 1 , mortality was higher among those patients with exercise induced ST depression(12 of 74), than among those without (13 of 256, $\mathrm{p}<0.01$ ); exercise angina and exercise duration were not predictive. In group 2 , mortality was greater in those patients who only reached stage 3 or less of a modified Bruce exercise protocol (19 of 288 ), compared with those who attained stage 4 or more (4 of 266, p < 0.01). Neither exercise angina nor ST depression was predictive in this group. A history of severe angina was not associated with adverse prognosis in either group. In the groups at higher risk there was no difference in mortality between those treated medically and those treated surgically.

SVD patients have a higher mortality than those without significant coronary disease. High risk subgroups can be identified by means of exercise testing and the angiographic assessment of $\mathrm{LV}$ function.

\section{Restenosis after coronary angioplasty: an analysis of stenosis morphology and the efficacy of repeat angioplasty}

\author{
R Henderson, A Pipilis, A Timmis, E Sowton \\ Guy's Hospital, London
}

Restenosis following successful percutaneous transluminal coronary angioplasty (PTCA) is an important clinical problem. Indeed in some patients PTCA may appear to accelerate the stenotic process.
We have therefore examined stenosis morphology at restenosis, and reviewed the efficacy of repeat PTCA. Following 328 successful PTCA procedures, restenosis (to $>50 \%$ luminal diameter) was found in 70 patients. Angiographic records were available for retrospective analysis in 42 of these patients. The measured diameter stenosis before PTCA was $74(12 \%)$ (mean (SD)) compared with $72(14 \%)$ at restenosis (NS). The stenoses, however, were significantly longer at restenosis than at the initial procedure $(8.6(3.7)$ and $6.7(2.4) \mathrm{mm}, \mathrm{p}<0.01)$. Furthermore in 52 patients who underwent repeat PTCA the trans stenotic pressure gradient was greater at restenosis than at the initial procedure (63 (26) and $56(19) \mathrm{mm} \mathrm{Hg}, \mathrm{p}<0.01)$. The angiographic success rate of repeat PTCA was $86 \%$. Procedure related complications were two myocardial infarcts, one referral for emergency surgery, and one death.

These data indicate that at restenosis after PTCA lesions tend to be longer, suggesting that vascular trauma at the initial procedure often extends beyond the limits of the coronary stenosis. Nevertheless repeat PTCA can be performed with a high degree of success and without excessive complications.

\section{Epicardial electrogram during angioplasty}

P Taggart, P M I Sutton, R Hayward,

R W Emanuel, R H Swanton

Middlesex Hospital, London

Endocardial recordings of monophasic action potentials (MAPs) provide a sensitive index of localised myocardial ischaemia registering changes during pacing to the angina threshold before the appearance of changes in the ECG or the onset of chest pain. Positioning of a MAP catheter on the left ventricular endocardium in the area of interest is not always technically easy or practical. We have appraised a MAP catheter on the right ventricular septum and an electrogram recorded from the angioplasty guide wire in the epicardial coronary artery. During balloon occlusion the electrograms registered ST depression and $T$ wave changes that were readily quantifiable. Changes in the repolarisation of the MAP varied depending upon the position of the catheter. Our findings showed that the electrogram is useful in providing a sensitive, good quality, and easily obtainable signal using the guide wire which is already in position. The recording site, however, is limited to the course of the epicardial artery.

The MAP catheter enables examination of the electrophysiological changes in areas of the heart not 
directly influencing the electrogram. The combination of both the MAP and the electrogram, however, allows a flexible and comprehensive investigation of the myocardium during angioplasty.

\section{Surface ventricular late potential activity in sustained and transient ventricular arrhythmias}

\author{
Susan Lewis, P I Lander, Penelope Taylor, \\ D A Chamberlain, $R$ Vincent \\ Royal Sussex County Hospital, Brighton
}

Surface ventricular late potential activity (VLPA) provides non-invasive evidence of slow conduction which is one of the prerequisites for reentry. We examined VLPA in 108 patients to study the mechanism of sustained and transient ventricular arrhythmias. Sixty two patients had recurrent sustained ventricular tachycardia that degenerated in some cases to ventricular fibrillation (group I), 21 suffered ventricular fibrillation during acute myocardial ischaemia (group II), 20 had non-sustained salvos of ventricular tachycardia (group III), and five had frequent premature ventricular complexes (group IV). Eighty one of the 108 patients had had previous myocardial infarctions, 12 had other cardiac disease, and 15 had "normal" hearts. VLPA occurred frequently in group I (50 of $62,81 \%$ ), but was less common in group II (7 of $21,33 \%, p<0.001)$, in group III (3 of 20,15\%,p $<0.0001$ ) and in group IV ( 1 of $5,20 \%$, NS). Though evidence of slow conduction was found in most patients with sustained ventricular arrhythmias, it was not found commonly in patients with ventricular fibrillation and acute ischaemia, in those with non-sustained ventricular tachycardia, or in those with premature ventricular complexes.

Reentry may not, therefore, account for these transient ventricular arrhythmias. A difference in the mechanism of arrhythmogenesis may have important therapeutic implications.

\section{Chronic second degree block: a comparison of surface ECGs, His bundle electrograms, and effort tests}

N J Linker, C A Kekwick, A Whistance, D B Shaw Cardiac Department, Royal Devon and Exeter Hospital, Wonford

In 1982 we presented evidence to the society that chronic Wenckebach second degree block was not as benign as had always been thought. Subsequently it has been suggested that the standard ECG is inadequate for assessing the site of $\mathrm{AV}$ block and that His electrograms and exercise tests are required. We present data in patients seen in the Devon Heart Block and Bradycardia Survey. Eighty one patients were studied; 59 figured in our previous survival data and 22 were investigated subsequently. All had chronic second degree block, there were 26 with Wenckebach block (group I), 34 with Mobitz II block (group II), and 21 with fixed 2:1 or 3:1 block (group III). Fifty three patients had His electrograms, 38 being from groups I and II. In these the conduction delay was at the expected level in 30 . It was paradoxical in three, multisite in five, and split His potentials were seen in two. A prolonged $\mathrm{HV}$ was the predominant finding in 15 patients from group III but a split His potential was seen in four. Fifty six patients had exercise tests, 45 coming from groups I and II. In these the change in conduction was in the expected direction in 17 and the reverse in three, but in 25 no change was recorded. In 11 patients from group III, conduction improved in one, deteriorated in six, and was unchanged in four.

In our patients the site of block indicated by the surface ECG usually coincided with that found on the His electrogram, and intrahisian block may mimic AV nodal delay but was only found in $10 \%$ of cases. Exercise tests proved of limited value as no change in conduction was recorded in about half of the patients.

\section{Detection of superior limb and mid-baffle obstruction after Mustard's operation using spectral analysis of jugular venous Doppler signals}

Frances Soul, T K Hames, G R Sutherland, B R Keeton

Wessex Regional Medical Physics Department and Wessex Cardiothoracic Unit, Southampton

Venous pathway obstruction after Mustard's operation occurs in about $10 \%$ of patients. It is usually suspected clinically and confirmed by angiography. Diagnosis using pulsed Doppler interrogation of the pathways and examination of the zero crossing output of the jugular venous Doppler signal has been reported. In view of inherent inaccuracies in the zero crossing technique we have used spectral analysis to evaluate the jugular venous Doppler waveform and its timing in association with the common carotid arterial signal obtained at the same site and displayed 
on a flow direction sensitive display. We have examined 47 of 67 survivors of Mustard's operation since 1974. Twelve have had correlative catheterisation including nine with baffle obstruction examined before and after relief by balloon angioplasty or surgery. In cases without evidence of obstruction the jugular venous signal has a biphasic waveform, the second component larger than the first, occurring synchronously with the diastolic phase of the carotid arterial signal. In nine patients with baffle obstruction (eight-superior limb, one-mid baffle) there was a change in the timing of the maximum venous component, becoming synchronous with the systolic peak of the carotid arterial signal, which normalised after relief. Severe venous turbulence $(n=14)$ was not an indicator of baffle obstruction. Spectral analysis of the jugular venous Doppler signal is a simple, rapid, reliable technique for detection of baffle obstruction. It has enabled us to detect superior limb obstruction in patients in whom it was clinically not suspected and at a stage when it was not so severe as to preclude balloon dilatation.

Ebstein's anomaly diagnosed in the first year of life: factors affecting mortality

P A Russo, R K H Wyse, F Triumbari, Susan Dodd, $M$ R de Leval

Thoracic Unit, Hospitals for Sick Children, Great Ormond Street, London

The outcome of 42 patients with Ebstein's anomaly symptomatic during the first year of life (mean age 6 days) was reviewed. All had tricuspid regurgitation. Congestive heart failure (CHF) and cyanosis were present in 22, cyanosis alone in 13, CHF alone in one, and serious arrhythmias in five. Systemic to pulmonary shunts were performed in seven, tricuspid annuloplasty in one (at 90 days), and tricuspid valve replacement (at 17 months) in one. Survival was $69 \%$ at 14 days, $52 \%$ at one year, and $37 \%$ at five years. Thirteen patients died within two weeks of diagnosis (mean age 5 days) all due to. CHF or hypoxia. In the 14 patients who died later (mean age 2.9 years) sudden death occurred in eight. There were four surgical deaths ( $9 \%$ ) (mean age 8 months). Univariate analysis of the clinical, anatomical, and histological factors leading to early death showed that age at presentation, hypoplasia - absence of trabecular and outlet portion of the right ventricle or both-small tethered leaflet, and associated anomalies were independent risk factors for early mortality. Quantification by computerised morphometric anal- ysis showed that the right ventricular free wall fibrous content was significantly raised $(p<0.001)$ compared with controls matched by age and size. Neither reduced wall thickness nor the increased right ventricular fibrous content, however, affected the time of death.

Early death is therefore strongly influenced by morphological but not histological features. Patients with favourable anatomy, however, are also at risk of sudden death, probably due to arrhythmias.

\section{Peripheral pulmonary vascular and airway abnormalities in Indian adolescents with rheumatic mitral stenosis}

Sheila Haworth, S M Hall, M Patel

Department of Paediatric Cardiology, Institute of Child Health, London, and Institute of Postgraduate Medical Education and Research, Calcutta, India

Lung biopsy specimens were taken from 15 adolescents (aged 11-21 years), when a mitral valvotomy was performed in Calcutta. They were examined by light, transmission, and electron microscopy. Abnormalities were most pronounced in the capillaries. They were embedded in dense connective tissue, and an increase in basement membrane material thickened the blood gas barrier. Intra-acinar arteries and veins showed an increase in muscularity and in adventitial connective tissue, with severe circumferential intimal fibrosis. Airway abnormalities were striking. The peripheral airways were encased and compressed by dense connective tissue and bronchial smooth muscle was increased. Six months after the mitral valvotomy was performed, the mean pulmonary arterial pressure had fallen from 42 to $23 \mathrm{~mm} \mathrm{Hg}$. Despite this satisfactory haemodynamic result, the long term effects of small airway compression on the growing lung indicate the need for early relief of mitral valve obstruction.

Adults with mitral stenosis may have persistent respiratory problems after valvotomy: Given our findings in young people, this may perhaps be attributed to the long term effects of the heart disease rather than chronic bronchitis as is often supposed.

\section{Diastolic filling dynamics after intravenous verapamil in coronary patients}
I Amende, $\mathbf{R}$ Simon, A Seegers, $\mathbf{P}$ R Lichtlen
Division of Cardiology, Hannover Medical School, West Germany 
We studied the effects of intravenous verapamil (V) on left ventricular (LV) pump function and diastolic filling dynamics. We gave $\mathrm{V}(0 \cdot 1 \mathrm{mg} / \mathrm{kg}$ bolus followed by $0.005 \mathrm{mg} / \mathrm{kg} / \mathrm{min}$ ) to 10 patients four years after coronary bypass grafting and implantation of seven intramyocardial tantalum markers (MM). The studied patients had severe coronary artery disease. We simultaneously measured LV systolic (LVSP) and end diastolic pressure (EDP), positive $\mathrm{dP} / \mathrm{dt}$ and $\tau$, the time constant of isovolumetric pressure decay, by tipmanometry; cardiac index $(\mathrm{CI})$ and coronary sinus blood flow (CSF) by thermodilution; and end diastolic volume (EDV), ejection fraction (EF), and peak diastolic filling rate (PFR) by MM filmed at 100 frames/second (biplane $30^{\circ} \mathrm{RAO} / 60^{\circ} \mathrm{LAO}$ ). Pressure volume relations were obtained in early, mid, and end diastole. $\mathrm{V}$ caused a slight but significant rise in $\operatorname{EDP}(11$ to $14 \mathrm{~mm} \mathrm{Hg}$ ) and EDV (142 to $152 \mathrm{ml}$ ) and a fall in positive $\mathrm{dP} / \mathrm{dt}$ (1748 to $1640 \mathrm{~mm} \mathrm{Hg} / \mathrm{s}$ ), reflecting the drug's negative inotropic action. CI (2.7 and $2.91 /$ minute) and EF (47 and $49 \%$ ) did not decline due to LV unloading. Peripheral resistance decreased significantly (1496 and 1348 dyn s cm${ }^{-5}$ ) and CSF increased (88 vs $102 \mathrm{ml} /$ minute). The speed of relaxation (63 and $62 \mathrm{~m}$ seconds) remained unaltered, and the pressure volume relation was shifted on the same curve to slightly higher values. PFR, however, was enhanced (269 to $334 \mathrm{ml} /$ seconds).

Thus verapamil has little effect on systolic pump function, relaxation, and diastolic distensibility of the LV, but improves early diastolic filling. This improvement seems to be associated with an alteration in loading conditions due to peripheral vasodilatation or in part to a reduction in myocardial ischaemia due to an increase in coronary flow.

\section{British regional heart study: an ECG assessment of risk}

P W Macfarlane, A Phillips, Mary Walker, S J Pocock, A G Shaper

University Department of Medical Cardiology, Royal Infirmary, Glasgow, and the Department of Clinical Epidemiology and General Practice, Royal Free Hospital, London

As part of the British regional heart study, ECGs were recorded on 7735 men aged between 40 and 59 years. These ECGs were analysed by computer and coded results were transferred to a separate data base for statistical analysis. Follow up data on morbidity and mortality have now been obtained on each subject for at least five years and in some cases, up to seven and a half years. A new major ischaemic event-that is, acute myocardial infarction (MI), fatal or non-fatal-or sudden death was detected in 336 men during the follow up period. Non-fatal events accounted for over half the episodes $(63 \%)$. Thirty five of those whose first event was non-fatal sustained a subsequent fatal event. ECG evidence of a previous MI carried over a five fold risk of a patient sustaining a further event, definite ischaemia carried a threefold risk while possible infarction and possible ischaemia carried a twofold risk compared with those with ECGs without such abnormalities. In a similar fashion, patients with LVH and secondary ST-T changes had over five times the risk of a future incident, whereas LVH without ST-T changes carried a twofold risk compared with those without such evidence. Overall, patients with any of these ECG abnormalities had twice the risk of a future event compared to those without such ECG changes whether or not they had other symptoms of ischaemic heart disease from the history.

In view of the fact that a high percentage of cases of myocardial infarction are silent and the ECG alone indicates the presence of secondary ST-T changes, it is concluded that the resting ECG has an important role in population screening.

\section{Arrhythmias after recombinant tissue plasminogen activator in patients with acute myocardial infarction}

A J McNeill, S R Cunningham, J S Shannon, N P S Campbell, M M Khan, G C Patterson, S W Webb, A A J Adgey

Regional Medical Cardiology Centre, Royal Victoria Hospital, Belfast

Arrhythmias (A) in patients following attempted thrombolysis ( $\mathrm{T}$ ) with recombinant tissue plasminogen activator (rt-PA) in the early phase of myocardial infarction (MI) are rarely documented. We monitored the ECGs continuously of 50 consecutive patients with MI (41 men, nine women; mean age 59 years, range $42-71$ ) randomised to $20 \mathrm{mg}, 50 \mathrm{mg}$, or $100 \mathrm{mg} \mathrm{rt}-\mathrm{PA}$ on average $135 \mathrm{~min}$ after the onset of pain (range $20-240 \mathrm{~min}$ ), and given intravenously over 90 minutes. At 90 minutes infarct related vessel patency was assessed by coronary angiography and graded 0-3 (TIMI) reperfusion. Forty one patients received a further $50 \mathrm{mg}$ rt-PA over five hours and in seven it was over one hour. In 48 patients, intravenous heparin was given immediately after rt-PA. At the start of rt-PA all patients were in sinus rhythm although three had had third degree AV block when 
first seen. Following rt-PA 39 patients had $\geqslant 1$ major A during out of hospital and in hospital observation. Twenty patients had $\geqslant 1 \mathrm{~A}$ during the $90 \mathrm{~min}$ rt-PA infusion: four patients developed ventricular fibrillation (VF) (one during cardiac catheterisation), two patients had idioventricular rhythm (IDVR), self terminating ventricular tachycardia (self term VT, defined as five or more consecutive ventricular ectopics) and runs of ventricular bigeminy in eight, atrial fibrillation (AF) in two patients, transient 2:1 AV block in one patient, nodal rhythm (NR) in three patients, and sinus bradycardia in two. Five of these 20 patients received $20 \mathrm{mg}$ rt-PA, nine $50 \mathrm{mg}$, and six $100 \mathrm{mg}$ : eight had grade 3, four-grade 2, fourgrade 1 and four-grade 0 reperfusion. Of the seven patients subsequently receiving $50 \mathrm{mg}$ rt-PA over one hour, one developed IDVR and NR. Of the 41 patients receiving it over five hours, two patients developed VF, nine IDVR, and six self term VT. Between the end of rt-PA and discharge from hospital 12 patients had self term VT, nine patients had IDVR, and three AF. Late VF did not occur and 46 survived hospital treatment.

The overall incidence of VF $(12 \%)$ is similar to that in patients with MI not undergoing thrombolysis.

Factors influencing the perception of pain in exercise induced myocardial ischaemia after acute myocardial infarction

Leisa Freeman, Jenny King, P G F Nixon

Cardiac Department, Charing Cross Hospital, London

Eighty five patients underwent modified Bruce protocol exercise testing two weeks and three months after documented myocardial infarction. Twenty five of $85(30.1 \%)$ had ST segment depression ( $>1 \mathrm{~mm}$ ) on both occasions. Chest pain (CP) accompanied the ECG changes in 13 of $25(13.2 \%)$; in 12 of $25(15.7 \%)$ ST depression was asymptomatic (ASY). No patient was diabetic. Baseline measurements of age, sex, smoking, site of infarction, ejection fraction, and enzyme rises were not significantly different among the groups. At two weeks, exercise length was 8.5(4.9) (CP) and $10.0(3.3)$ (ASY); at three months, exercise length was $10.0(5.1)$ and $13.9(1.9), \quad(p<0.06)$, respectively. Patients with angina scored significantly higher on an illness behaviour questionnaire $(p<0.03)$ and in particular on the hypochondriasis index $(p<0.05)$ and anxiety depression scales $\quad(p<0.03)$. Forced hyperventilation reproduced chest pain in seven patients, six with exercise induced chest pain and one without $(\mathrm{p}<0.05)$; there was a greater mean fall in $\mathrm{PetCO}_{2}$ at two weeks but not at three months on approach to exercise in patients who subsequently sustained chest pain. A significantly higher life event stress score in the months before infarction was found in the subjects with chest pain $(p<0.04)$.

Patients who perceive pain on exercise with ST segment depression recognise more life events as stressful and have a greater anxiety and somatic awareness than patients who do not. Interestingly patient assessment of quality of life was not significantly different although relative assessment was persistently lower in the chest pain group.

Clinical and angiographic predictors of acute coronary occlusion at coronary angioplasty

A Seth, R A Perry, M F Shiu

University Department of Cardiovascular Medicine, Queen Elizabeth Hospital, Birmingham

Acute coronary occlusion is a major complication of coronary angioplasty (PTCA) and if not successfully redilated leads to myocardial infarction (MI) or necessitates coronary artery bypass grafting (CABG). To identify risk factors for this complication in a consecutive series of 234 patients undergoing PTCA we have analysed retrospectively the clinical features and coronary stenosis morphology (length, severity, and eccentricity) of 29 patients who had acute occlusion at PTCA (group I), of whom 15 went on to develop a procedure related MI. Twenty nine patients matched for target vessel disease who underwent an uncomplicated procedure served as controls (group II). Group I had significantly longer coronary stenoses than group II (mean length $13.6 \mathrm{~mm}$ and $10.3 \mathrm{~mm} ; \mathrm{p}=0.005$ ). The severity of stenoses was similar in both groups as was the incidence of eccentricity. In the subgroup of patients, however, who could not be successfully redilated and therefore had a procedure related $M I$, there was a significantly higher number of eccentric stenoses compared with those who were successfully redilated (nine of 15 and two of $14 ; p<0.02$ ). This group also had a significantly higher number of patients with remote previous MI (13 and five; $p<0.01$ ).

We conclude that longer stenoses are more likely to occlude at PTCA, and if eccentric, redilatation is frequently unsuccessful leading to a procedure related $M I$. The risk of this occurring is also high in presence of a previous remote MI. Risk assessment before PTCA should take into account the above angiographic and clinical characteristics. 


\section{Renin angiotensin aldosterone system and posture in the response to diuretics in cardiac failure}

A D Flapan, S Stewart, A Gill, C Waugh, E Davies, B C Williams, T R D Shaw, C R W Edwards

Departments of Medicine and Cardiology, Western General Hospital, Edinburgh

Bed rest promotes a diuresis in patients with oedema and the response to intravenous diuretics is greater in the supine position compared with the upright position. It is thought that this effect is due to the increased activity of the renin angiotensin aldosterone system during upright posture. To test this hypothesis we have studied the effect of bumetanide $1 \mathrm{mg}$ given intravenously to 10 patients with cardiac failure established on enalapril, in the upright and supine positions. Control blood samples were drawn for the measurement of plasma renin activity and plasma concentrations of angiotensin II and aldosterone after one hour of supine rest. Subjects then emptied their bladders and were given bumetanide $1 \mathrm{mg}$ intravenously. They were then assigned to upright or supine posture, and all urine was collected for the next four hours and further blood sampling took place at 60,120 , and 240 minutes after the injection. Each subject had studies in both upright and supine positions. With the subjects supine urine output was 834 (94)'ml and:sodium excretion $85(14.5) \mathrm{mmol}$; when upright urine output was slightly lower at $728(99) \mathrm{ml}(\mathrm{p}>0.05)$, and sodium excretion was $76(15 \mathrm{mmol})(p>0.05)$. Glomerular filtration rate was lower in the upright $(86(24) \mathrm{ml} / \mathrm{minute})$ than in the supine position (112(24) $\mathrm{ml} / \mathrm{minute})(\mathrm{p}>0.05)$. There was no difference in plasma renin activity and angiotensin II concentration in either position even though there was an increase in plasma aldosterone in the upright position.

We conclude that converting enzyme inhibition blocks the expected effect of posture on diuretic therapy.

\section{Subtherapeutic dosing of flecainide as a provocative test for non-inducible ventricular tachycardia}

D Mehta, D E Ward, A J Camm

St George's Hospital Medical School, London

Induction of clinical ventricular tachycardia (VT) is important during an electrophysiology study in order to map its origin. Flecainide acetate at low blood concentrations shortens the effective refractory period of Purkinje fibres but prolongs that of the ventricular muscle. This disparate change in effective refractory period may exaggerate heterogeneity in recovery of excitability and allow reentry. Slowing of conduction by flecainide may perpetuate reentry in susceptible patients. Eleven patients (mean age 56 years, range 19-70) with previously documented sustained VT (cycle length $343(62)$ mean (SD)), range $280-480 \mathrm{~ms}$ were studied. A 12 lead electrocardiogram during clinical tachycardia in seven patients showed left bundle branch configuration and in three a right bundle branch pattern. In one patient clinical VT was multiform. The ventricular stimulation protocol, consisting of sinus rhythm or a drive of 100,120 , or $140 \mathrm{bpm}$ plus one, two, or three ventricular extrasystoles delivered to the right ventricular apex, did not provoke arrhythmia in any patient. Flecainide acetate (1 $\mathrm{mg} / \mathrm{kg}$ ) was given intravenously over five minutes. The stimulation protocol was repeated 10 minutes after the start of the administration. In four (36\%) patients sustained VT could be induced by a drive cycle (in two patients at 120 and other two at 140 bpm) and two ventricular extrasystoles. In three patients the configuration and mean rate of induced VT, as identified by a 12 lead electrocardiogram, was identical to the previously documented spontaneous clinical VT. In one patient a faster non-clinical arrhythmia was induced. With this dose of flecainide acetate the ventricular effective refractory period increased from $243(21)$ to $256(22) \mathrm{ms}(\mathrm{p}<0.01)$.

Thus, in patients with VT in whom routine electrical stimulation fails to provoke arrhythmia, repeat stimulation after a subtherapeutic doses of flecainide might be used as a provocative test.

Serum cortisol concentrations in myocardial infarction are predictive of prognosis

\section{R J I Bain, J Jagger, J P Fox, M K Davies, R G Murray, W A Littler \\ Department of Cardiovascular Medicine, East \\ Birmingham Hospital, Birmingham}

Serum cortisol concentrations are raised in acute myocardial infarction but not in angina. We have studied prospectively the time course of the cortisol, creatine kinase (CK), and CK-MB response to myocardial infarction. In 70 patients admitted within six hours of an acute myocardial infarction blood was taken at presentation, then four hourly over the next 36 hours and then at 48,60 , and 72 hours. The samples were centrifuged and serum stored at 
$-70^{\circ} \mathrm{C}$. Serum cortisol concentrations were highest on admission $968(340) \mu \mathrm{mol} / 1$ (mean (SD)) and fell slowly over the next two days. Serum CK and CK$\mathrm{MB}$ concentrations at presentation were $318(668)$ $U / 1$ and $36(63) U / 1$ respectively and reached peak concentrations at $12-16$ hours $(2486(1627)$ and $334(224) \mathrm{U} / 1$ respectively). The cortisol and CK-MB concentrations correlated poorly at presentation $(r=0.22)$. However, peak $C K-M B$ correlated with peak cortisol concentrations $(r=0.53, p<0.001)$. Five patients died within 10 days. There was no difference in initial $(36(63)$ and $36(23) \mathrm{U} / \mathrm{l})$, or peak $(334(224)$ and $559(322) \mathrm{U} / \mathrm{l})$ $\mathrm{CK}-\mathrm{MB}$ concentrations between those surviving and those dying. In those who died, however, cortisol concentrations were significantly higher both at presentation, $1448(423)$ and $931(309) \mu \mathrm{mol} / \mathrm{l}$, $(\mathrm{p}<0.01)$ and at peak levels, $2317(795)$ and 1094 (450) $\mu \mathrm{mol} / 1,(p<0.001)$. Only one patient who died had a peak cortisol below $2000 \mu \mathrm{mol} / \mathrm{l}$. Of the survivors only two had peaks above $2000 \mu \mathrm{mol} / 1$, giving this concentration predictive value for mortality of $60 \%$, sensitivity $80 \%$, specificity $96 \%$, and an accuracy of $95 \%$.

Serum cortisol concentration is raised earlier in the course of infarction than $C K$ and $C K-M B$ and shows a greater predictive value for prognosis. These features may be of value in early intervention studies in myocardial infarction.

\section{Enhanced granulocyte aggregation responses after acute myocardial infarction}

\author{
T C Fisher, J J F Belch, I McGhie, I Hutton, \\ Ann Tweddel \\ University Department of Medical Cardiology, \\ Royal Infirmary, Glasgow
}

Raised white cell count is an accurate predictor of myocardial infarction (MI) and stroke, and also correlates with risk of recurrence and mortality in these diseases. Thus it has been proposed that leucocytes have an important role in the pathogenesis of ischaemic vascular disease. Using a recently developed whole blood aggregation assay, granulocyte function in 12 patients with acute $\mathrm{MI}$ was compared with results from 12 normals matched for age, sex, and smoking habit. White cell count (WCC) and differential were also measured. Samples were taken within six hours of onset, and MI was confirmed by ECG and enzyme changes. The aggregation response of granulocytes to the chemotactic tripeptide nFMLP $(40 \mu \mathrm{mol} / 1)$ was significantly greater in the MI group $(\mathrm{p}<0.0001)$, as were the total
WCC, and the granulocyte count (both $\mathrm{p}<0.0001$ ). No correlation between WCC and aggregation was observed, and adjusting the granulocyte count by diluting with buffy coat free blood did not reduce the granulocyte response. Thus blood from MI patients contains large numbers of aggregatable granulocytes. Such cells are liable to become sequestered in the microcirculation promoting ischaemia by physically impeding flow and causing tissue damage by the release of toxic oxygen metabolites.

Whether this is of aetiological importance is uncertain but it is plausible that further myocardial damage will be caused in this manner after the acute event. Infarct size has been measured as part of the study and will be compared with the granulocyte function data.

\section{Ischaemic left ventricular dysfunction: frusemide or nitrate?}

Ann Tweddel, W Martin, I McGhie, I Hutton University Department of Medical Cardiology, Royal Infirmary, Glasgow

In patients with myocardial ischaemia the acute effects of intravenous (IV) frusemide, with increased systemic vascular resistance may be detrimental, and treatment with nitrate may be more appropriate. Eighteen patients were studied with ischaemic left ventricular dysfunction (EF less than $30 \%$ ); eight randomly received frusemide $40 \mathrm{mg} \mathrm{IV}$, and ten isosorbide mononitrate (ISMN) $15 \mathrm{mg}$. Coronary and systemic haemodynamics were measured and myocardial nutrient flow assessed by xenon washout. With ISMN blood pressure was unaffected, whereas with frusemide systolic, diastolic, and mean arterial pressure increased at $30 \mathrm{~min}$ (91 (4) to $104(6) \mathrm{mm} \mathrm{Hg}$ ). Because cardiac index fell at $30 \mathrm{~min}$ with ISMN $(2.71(0.12)$ to $2.33(0 \cdot 17),(p<0.02))$ and was unchanged with frusemide $(2 \cdot 55(0 \cdot 13)$, $2 \cdot 53(0.14) 1 /$ minute $\left./ \mathrm{m}^{2}\right)$, with both treatments the calculated systemic vascular resistance increased, with ISMN by $17 \%(1578(186)$ to $1844(255)$, $\mathrm{p}<0.05)$ and with frusemide $14 \%(1574(148)$ to $1786(180)$ dyn.s.cm $\left.{ }^{-5}, p<0.02\right)$. Nitrate produced no significant change in coronary sinus flow $(119.7(13.1)$ to $108(17.7) \mathrm{ml} / \mathrm{minute})$ nor in myocardial nutrient flow, but oxygen extraction decreased with a narrowing arteriovenous oxygen difference $(11.9(0.83), 10.4(0.68)$, and $10.7(0.73)$ vol $\%$ at 30 minutes, $p<0.02$ ). In contrast, with frusemide coronary sinus flow increased $(130.3(11.6)$ to $170(25) \mathrm{ml} /$ minute, $\mathrm{p}<0.05)$, extraction was unchanged and myocardial nutrient 
flow increased from the left coronary injection $(54.7(5.4)$, to $67 \cdot 6(4), p<0.05)$ but was unchanged for the right coronary injection $(45 \cdot 4(6 \cdot 6)$ to $39 \cdot 2(4 \cdot 8) \mathrm{ml} / \mathrm{minute})$.

These results suggest that despite the increased systemic vascular resistance, coronary haemodynamic function is not adversely affected by frusemide and there would be little advantage in using nitrate.

\section{Post cardioversion ventricular arrhythmias in patients taking digoxin}

D Gilligan, D Kenny

Royal City of Dublin Hospital, Ireland

Clinical and experimental evidence suggests a risk of inducing malignant ventricular arrhythmias when digoxin toxicity is present. Recent studies did not show any increase in ventricular arrhythmias if the serum level of digoxin was normal. It has been inferred that in the absence of evidence of digoxin toxicity, cardioversion could proceed safely. To test this hypothesis a retrospective study was carried out comparing the occurrence of ventricular arrhythmias in a group of patients on digoxin at the time of cardioversion with a group who were not (controls). Eighty three elective direct current cardioversions were performed for atrial tachyarrhythmias $(80 \mathrm{men}$, mean age 54 years). Fifty patients were taking digoxin (40 taking $0.25 \mathrm{mg}$ daily) and received their last dose 23(14) hours before cardioversion. None had clinical or electrocardiographic evidence of toxicity, and digoxin concentrations were not measured. Immediate post cardioversion ventricular arrhythmias (bigemini, multifocal ectopics, and nonsustained ventricular tachycardia) occurred in 10 of the 50 patients on digoxin and one of the 33 controls, $p<0.05$. Multivariate analysis of other variables showed that digoxin was independently correlated with the occurrence of arrhythmias, $p<0.05$. Arrhythmias tended to occur more frequently in patients on digoxin $0.5 \mathrm{mg}$ daily or if the last dose was within 12 hours of cardioversion, $p=$ NS.

There was a higher incidence of potentially malignant ventricular arrhythmias following cardioversion in patients on digoxin compared with controls. Caution is advised when cardioverting digitalised patients even those apparently non-toxic.
Changes in left ventricular performance after coronary artery surgery

C E Handler, A Pipilis, $N$ Hamouratidis, E Sowton, $P$ Deverall, A Yates

Guy's Hospital, London

The serial effects of myocardial revascularisation on left ventricular performance were assessed using echocardiography and symptom limited treadmill exercise testing in 54 patients. Before coronary artery surgery (CAS) 35 patients had had at least one myocardial infarction, 19 had moderate, and nine had severely abnormal left ventricular function shown angiographically; 48 patients had multivessel coronary artery disease. Up to six months after CAS, there were no significant changes in left ventricular end diastolic diameter although percentage left ventricular fractional shortening decreased significantly at one month $(31.0 \%$ and $28.8 \%, p<0.05)$, but not thereafter. There was a significant increase in the incidence of new left ventricular wall movement abnormalities after CAS; $77 \%$ of the patients developed anteroseptal hypokinesia with coexistent posterolateral hyperkinesia 10 days postoperatively, but the frequency of this decreased to $31 \%$ by six months. Exercise haemodynamics, exercise duration, and features of reversible myocardial ischaemia all improved progressively and significantly after CAS. No patient sustained a CAS related myocardial infarction and there were no changes in electrocardiographic $\mathbf{R}$ wave amplitudes during follow up.

We conclude that new abnormalities in left ventricular wall movement develop quite commonly early after CABG but usually resolve by six months and do not seem to influence exercise performance or haemodynamics.

\section{Evaluation of myocardial tomography by thallium-201 in patients with chest pain}

C J Foster, G P Lawrence, D L Hastings, M C Prescott, $\mathrm{H}$ J Testa

Departments of Cardiology and Nuclear Medicine, Manchester Royal Infirmary, Manchester

Exercise thallium imaging is an accepted technique for the evaluation of patients with chest pain suspected of having coronary artery disease. The application of tomography to this technique should further increase its accuracy. Thirty two patients 
undergoing coronary arteriography for symptoms suggestive of angina have been studied using exercise tomographic thallium scanning. Patients were exercised to a symptom limited end point on a bicycle ergometer. ECG monitoring and recording was performed throughout. Seventy five $\mathrm{MBq}$ of thallium-201 was injected one minute before the end of exercise. Tomographic imaging was performed immediately following exercise. Thirty two images were acquired over a $180^{\circ}$ arc (time per image 40 seconds) and displayed on a $64 \times 64$ matrix. Sections were reconstructed parallel to and perpendicular to the long axis of the left ventricle. Fifteen patients had ECG evidence of previous myocardial infarction; thallium tomography identified 12 of these, and incorrectly identified seven further patients (sensitivity $80 \%$ specificity $59 \%$ ). Twenty five of the 32 patients had angiographic evidence of coronary disease not related to infarct areas; thallium tomography identified all of these and incorrectly identified two normal subjects as having a reversible defect (sensitivity $100 \%$ specificity $71 \%$ ). It was also possible to identify correctly which coronary arteries were affected. Thallium tomography correctly identified 46 major coronary stenoses but did not detect nine less severe lesions.

Exercise thallium tomography is an accurate technique for identifying patients with coronary artery disease and can be used to identify which coronary arteries are diseased.

\section{Magnetic resonance measurement of aortic compliance}

R Mohiaddin, S R Underwood, H Bogren,

D N Firmin, R H Klipstein, R S O Rees,

D B Longmore

Magnetic Resonance Unit, National Heart and Chest Hospitals, London

Arterial compliance has previously been difficult to measure non-invasively, and its importance in the assessment of vascular disease and as a component of left ventricular afterload is unknown. We have used magnetic resonance to study changes with age in regional aortic compliance and in total arterial compliance. Twenty normal volunteers were studied, age range 16 to 71 . Sections $10 \mathrm{~mm}$ thick were taken in three oblique planes perpendicular to the mid points of the ascending aorta (AA), the aortic arch, and the descending thoracic aorta (DA). Volume changes between diastole and systole were calculated from the change in area, and compliance was derived using the pulse pressure measured with a sphygmomano- meter. Total arterial compliance was calculated from the left ventricular stroke volume measured by summing chamber areas in multiple contiguous slices. Mean (SD) normal compliance in $\mathrm{ml} / \mathrm{mm} \mathrm{Hg}$ was: AA $0.031(0.018)$, arch $0.020(0.012)$, DA $0.014(0.007)$, and total compliance $1.46(0.35)$. Ascending aortic compliance was significantly higher than at the other sites $(p<0.001)$. There was a strong correlation between compliance and age for all three sites $(r=-0.76 \mathrm{AA},-0.83 \mathrm{arch}$, and $-0.71 \mathrm{DA})$, but there was decreasing dependence upon age more distally (slope $=-77 \mathrm{AA},-56 \mathrm{arch}$, $-38 \mathrm{DA}, \times 10^{-5}$ ).

We conclude that regional aortic and total arterial compliance can be measured non-invasively by magnetic resonance. The ascending aorta is the most compliant region of the aorta and normal compliance falls with age. The importance of these results for patients with cardiovascular disease remains to be determined.

\section{Pulmonary artery blood flow patterns studied} by magnetic resonance

H Bogren, $\mathbf{R} \mathbf{H}$ Klipstein, $\mathbf{R}$ Mohiaddin, $S$ R Underwood, D N Firmin, R S O Rees, D B Longmore

Magnetic Resonance Unit, National Heart and Chest Hospitals, London

Pulmonary artery flow can be studied non-invasively by cine magnetic resonance velocity mapping, and it is potentially of interest in a variety of disorders. We have studied 25 normal subjects and three patients with pulmonary arterial hypertension, and compared their aortic flow and left ventricular output measured from ventricular volumes. Pulmonary artery distensibility has also been measured from changes in cross sectional area during the cycle. There was plug flow during most of systole with a small channel of reverse flow beginning just before pulmonary valve closure. The normal two dimensional velocity profile was skewed, but the direction of skew varied between individual subjects and throughout the cycle. Two centimetres above the pulmonary valve the dominant direction of the skew was posterior, but immediately below the bifurcation it was anterior. There was no regular pattern in two subjects. Pulmonary flow, aortic flow, and left ventricular stroke volume showed close agreement $(r=0.96, S E E=8 \mathrm{ml})$, validating the technique and suggesting a method for the measurement of intracardiac shunting. In the patients with pulmonary 
hypertension, pulmonary flow was reduced and there was an earlier and more extensive channel of reverse flow. Pulmonary artery distensibility (fractional change in area) was 0.07 (normal 0.25 to 0.30 ).

These studies validate magnetic resonance pulmonary artery velocity mapping and illustrate potential sources of error in Doppler flow measurements. The abnormalities in patients with pulmonary hypertension suggest that the technique may be of value in such patients, but futher studies are indicated.

\section{Cross sectional echocardiography in cardiac hydatidosis}

F Alfonso, M Rey, J Balaguer, R Rabago, $P$ Nihoyannopoulos, W J McKenna

Fundacion Jimenez Diaz, Madrid, and Royal Postgraduate Medical School, Hammersmith Hospital, London

Cardiac hydatidosis is rare, is curable by operation, and must be differentiated from cardiac tumours. We present the cross sectional echocardiographic findings in six patients with cardiac hydatidosis who subsequently underwent operation with pathological examination of the excised specimen. The patients were aged 20-59, median 37 years; there were five men and one woman. In addition, none had hepatic but five had pulmonary hydatidosis with pulmonary hypertension (mean pulmonary artery pressure $>36$ $\mathrm{mm} \mathrm{Hg}$ ). Echocardiography showed at least one large cyst in the left ventricular myocardium in five patients: in two this involved the anterior septum, in another two the cysts were in the inferior wall, and one of the patients had a grossly deformed apex of the left ventricle. In another patient the cyst was in the septum and had ruptured into the pericardial space. In the patient without left ventricular involvement, the cyst was attached to the posterior wall of the right atrium and had two pedunculated daughter vesicles which crossed the tricuspid valve into the right ventricle during diastole. Surgical pathology confirmed the echocardiographic appearances of non-homogeneous cavitations with calcifications.

Cross sectional echocardiography was useful in the identification and preoperative localisation of the cysts in these patients with cardiac hydatidosis.
Differential effects of acetylcholine on conductance and resistance vessels in patients with angiographically normal epicardial coronary arteries

C M H Newman, D R Hackett, H El-Tamimi, G J Davies, A Maseri

Royal Postgraduate Medical School, Hammersmith Hospital, London

Acetylcholine (ACh) has been shown to be a weak dilator of angiographically normal human coronary arteries in vivo, but constricts atherosclerotic segments. The effect of $\mathrm{ACh}$ on small resistance vessels has been less well documented. We studied eight patients aged 38-68 years with chest pain and normal coronary arteriograms but no myocardial ischaemia inducible by exercise or provocation tests for spasm. After diagnostic angiography, normal saline was infused at $1 \mathrm{ml} /$ minute into the left coronary artery for three consecutive two minute periods, followed by intracoronary $\mathrm{ACh}$ from $10^{-7} \mathrm{~mol} / 1$ to $5 \times 10^{-3}$ $\mathrm{mol} / \mathrm{l}$. Angiography was repeated after each infusion. Changes in left anterior descending (LAD) artery calibre were measured by computerised analysis. In three patients, changes in coronary blood flow during each infusion were assessed by the indirect Fick principle from changes in coronary sinus oxygen saturation $\left(\mathrm{CSO}_{2}\right)$. No significant changes in LAD calibre or $\mathrm{CSO}_{2}$ occurred with infusions of normal saline. ACh from $10^{-7}$ to $10^{-4} \mathrm{M}$ caused a small dose dependent dilatation of the proximal LAD in six patients, maximum $7 \cdot 2$ (6)\% (mean (SD), $\mathrm{p}=0.05$ ), but a large increase in $\mathrm{CSO}_{2}$ corresponding to an estimated flow increase of 367 (57)\%. From $10^{-3} \mathrm{~mol} / 1$ to $5 \times 10^{-3} \mathrm{~mol} / \mathrm{l}$, ACh caused diffuse constriction of the LAD in six patients, maximum $21.3(17) \%,(p=0.001)$ which was associated with angina and ischaemic ST changes. Constriction followed dilatation in four patients.

These data suggest that intracoronary $\mathrm{ACh}$ can cause both dilatation and constriction of the same segments of angiographically normal epicardial coronary arteries in man, dependent on dose. It also causes massive dose dependent increases in total coronary blood flow.

\section{Amiodarone: effects on colour vision}

D Travis, $\mathbf{P}$ Thompson, J Gunn, $\mathbf{R}$ M Boyle

Department of Psychology, University of York and York District Hospital, York

Amiodarone is a commonly prescribed drug used to 
control cardiac arrhythmias. An unwanted and universal side effect is corneal microdeposits visible with the slit lamp corneal microscope. An open question is whether amiodarone affects colour vision by either microdeposits or retinal insult. We studied the colour vision of 14 patients on amiodarone and 13 control patients. The tests of colour vision we used were those recommended by the international research group on colour vision deficiencies. The results show that there is no significant difference between the two groups on the Farnsworth-Munsell 100 hue test, the mean square root error scores (SD) being: control: 12.69 (5.21); amiodarone: 11.27 (4.23). On the more sensitive Pickford Nicolson anomaloscope, however, the amiodarone group required significantly $(p<0.05)$ more blue in the blue green match than the control group. This result would be expected in an acquired defect of colour vision showing a tritanopic (blue/yellow)axis. A correlation between time on amiodarone and the amount of blue required in the blue green match shows that the two variables are inversely correlated $(r=-0.68, p<0.02)$; - that is, the anomaloscope settings of the amiodarone group become more similar to those of the control group with increasing time on the drug. This suggests that amiodarone has an initial disruptive effect on colour vision that disappears over time (our results show that the critical duration is about twelve months).

We emphasise that the effects of amiodarone on colour vision are subtle and likely to be missed by most conventional tests of colour vision.

\section{Cardiac function and anatomy after Mustard's operation for transposition of the great arteries}

R S O Rees, Carol Warnes, Jane Somerville, S R Underwood, R Mohiaddin, D N Firmin, R H Klipstein, H Bogren, D B Longmore Magnetic Resonance Unit, National Heart and Chest Hospitals, London

Mustard's operation for transposition of the great arteries redirects the circulation at atrial level and it has been used successfully since 1964 . Many patients have now reached adult life, but ventricular function and tricuspid competence are important determinants of late morbidity. We have investigated magnetic resonance imaging in the assessment of cardiac anatomy and function in $\mathbf{1 7}$ adults, nine to 20 years after operation. The connections and anatomical associations of the great arteries were clearly visible in all cases. Five patients had residual ventric- ular septal defects which, with the exception of one small defect, were easily seen. The intra-atrial baffle was best seen in transverse slices, and the systemic venous connection showed as a fairly narrow channel lying in the posterior part of the cavity. The pulmonary venous connection was best seen in the coronal slices. Ventricular volumes were measured by adding areas in multiple contiguous sections, and the right to left ventricular stroke volume ratio was used as a measure of tricuspid regurgitation. Ejection fractions measured by radionuclide ventriculography were lower than the magnetic resonance measurements, and the correlation for the left ventricle was closer $(r=0.75)$ than for the right $(r=0.49)$. There was a significant difference between the mean right to left ventricular stroke volume ratio in those with Doppler echocardiographic evidence of tricuspid regurgitation $(1 \cdot 81)$ compared with those without $(1 \cdot 16)$, (normal range $0 \cdot 8$ to $1 \cdot 2$ ).

Magnetic resonance imaging is a useful adjunct in the postoperative follow up of patients who have had Mustard's operation for transposition of the great arteries.

\section{In vitro validation of assessment by Doppler ultrasound of the supra-annular (6650) and standard (6625) Carpentier-Edwards mitral prostheses}

J B Chambers, T Cochrane, M M Black, G Jackson Cardiac Unit, King's College Hospital, London

In native mitral valves, pressure half time $\left(T \frac{1}{2}\right)$ is fairly independent of flow compared with peak left ventricular inflow velocity (Vmax). This has not been validated for prosthetic valves. We therefore measured and compared $V \max , T \frac{1}{2}$, and actual orifice area (OA) for three unimplanted CarpentierEdwards valves (model 6650 sizes 31 and $29 \mathrm{~mm}$, model .6625 size $29 \mathrm{~mm}$ ) and one explant (model $662529 \mathrm{~mm}$ ). We used a computer controlled positive displacement pulse duplicator with 20 rate/stroke volume combinations (diastolic mean flow $3 \cdot 1-16.91 /$ minute). Doppler recordings were made with a Vingmed SD 50 and valve opening was recorded with a video camera. Vmax, $T \frac{1}{2}$, and OA all varied with flow. $T \frac{1}{2}$ was independent of stroke volume, but directly proportional to diastolic time interval. Alterations in the shape of the drive waveform, used to model ventricular compliance changes, affected $T_{\frac{1}{2}}$ independently of diastolic time interval. There was no significant difference in $T_{\frac{1}{2}}$ among the valves and $T \frac{1}{2}$ did not correlate with observed orifice area. Both Vmax and OA were directly proportional 
to rate and stroke volume and there was a correlation between $O A$ and $V \max$. The valves were significantly different in terms of $\operatorname{Vmax}(F=38$; $\mathrm{p}<0.0001)$ and $\mathrm{OA}(\mathrm{F}=12 ; \mathrm{p}<0.0001)$. Ranges for OA were $0.5-1.8 \mathrm{~cm}^{2}(665031 \mathrm{~mm}) ; 0.6-1.6 \mathrm{~cm}^{2}$ (6650 $29 \mathrm{~mm}) ; 0.4-1.6 \mathrm{~cm}^{2}(662529 \mathrm{~mm})$; and $0.3-1 \cdot 3 \mathrm{~cm}^{2}$ (explant $29 \mathrm{~mm}$ ).

For Carpentier-Edwards mitral prostheses in vitro, $T \frac{1}{2}$ is dependent on the shape and duration of the ventricular filling curve. This suggests that in vivo, $T \frac{1}{2}$ should be related to heart rate and ventricular function. Vmax is better than $T \frac{1}{2}$ as an indicator of orifice area.

\section{Determinants of cardiac reserve after intra-atrial repair of transposition of the great arteries}

J E Deanfield, T Cartwright, J Choi, M Griffin Hospitals for Sick Children, Great Ormond Street, London

Patients after intra-atrial repair of transposition of the great arteries are at risk of sudden death, especially during exercise. We used continuous wave Doppler to investigate the association of heart rate and stroke volume index during supine exercise in 36 patients without symptoms, 14 who had had a Senning operation [age range 6-15, mean 8.4 years] and 22 who had had a Mustard operation, age range 6.9-28, mean 15.9 years]). Resting heart rate, stroke volume index, and cardiac index were similar in both groups. The pattern of stroke volume index response during exercise was normal in the young patients ( $\leqslant 12$ years after surgery) with no significant differences between those who had had the Mustard and those who had had the Senning operation. In 16 older (Mustard) patients (11-23 years after surgery), however, cardiac index was significantly reduced $(p<0.05)$ due to a significant reduction in peak exercise stroke index volume $\left(43(8) \mathrm{ml} /\right.$ beat $/ \mathrm{m}^{2}$ in Mustard, 55 (4) $\mathrm{ml} /$ beat $/ \mathrm{m}^{2}$ in normal, $\mathrm{p}<0.001$ ). In eight of 16 patients stroke volume index fell by $20 \%$ or more compared to an average increase of $6 \%$ in normals. Heart rate responses were not-significantly different.

Older patients after the Mustard operation do not have stroke volume index reserve to maintain cardiac index during exercise. A precipitous fall in stroke volume index may develop during rapid heart rate, and place the patient at risk.

\section{Exercise capacity and symptoms are} objectively improved by an activity sensing pacemaker (Activitrax)

D P Lipkin, N Buller, M Frenneaux,

Lesley Ludgate, Trudy Lowe, S C Webb,

D M Krikler

Royal Postgraduate Medical School, Hammersmith Hospital, London

Atrial tracking pacemakers may improve exercise capacity and symptoms because they maintain atrioventricular synchrony and preserve the physiological heart rate response to exercise. A rate responsive pacemaker which reacts to "physical activity" (VVIActivitrax (Act)) may be effective in patients with sinus node disease who are unsuitable for VDD pacing. We performed a double blind randomised acute and chronic cross over study in 10 patients ages 30-75 years, at least three months after implant, who had complete atrioventricular block at rest and during exercise using a modified Bruce protocol. We assessed symptoms related to breathlessness, fatigue, and psychological state using a visual analogue scale questionnaire, and exercise capacity (maximal oxygen consumption and anaerobic threshold) during VVI-Act (peak rate 125/minute) and conventional fixed rate VVI pacing ( $70 /$ minute). One month after randomisation treadmill exercise was performed. The mode was then changed to the other pacing mode and exercise repeated three hours later. After another month the process was repeated but in the reverse order. During chronic assessment there was subjective improvement of breathlessness and fatigue with VVI-Act pacing $(p<0.05)$. During acute assessment maximal oxygen consumption $(\mathrm{ml} / \mathrm{kg} / \mathrm{minute})$ increased and the benefit was maintained during chronic VVI-Act and chronic VVI pacing: $18(1), 19(1)$, and $16(1),(p<0.02)$. Oxygen consumption ( $\mathrm{ml} / \mathrm{kg} / \mathrm{minute})$ at the anaerobic threshold likewise improved: 14 (1), 14 (1) and 12 (1), $(\mathrm{p}<0.04)$.

Activity detecting rate responsive pacing is thus superior to fixed rate ventricular pacing in patients with complete AV block. 